\title{
THE STRUCTURE OF ALGEBRAIC THREEFOLDS: AN INTRODUCTION TO MORI'S PROGRAM
}

\author{
JÁNOS KOLLÁR
}

\section{CONTENTS}

1. Introduction

2. What is algebraic geometry?

3. A little about curves

4. Some examples

5. Maps between algebraic varieties

6. Topology of algebraic varieties

7. Vector bundles and the canonical bundle
8. How to understand algebraic varieties?

9. Birational geometry of surfaces

10. Mori's program: smooth case

11. Mori's program: singular case

12. Flip and flop

13. Finer structure theory

Introduction. This article intends to present an elementary introduction to the emerging structure theory of higher-dimensional algebraic varieties. Introduction is probably not the right word; it is rather like a travel brochure describing the beauties of a long cruise, but neglecting to mention that the first half of the trip must be spent toiling in the stokehold. Perusal of brochures might give some compensation for lack of royal roads.

Having this limited aim in mind, the prerequisities were kept very low. As a general rule, geometry is emphasized over algebra. Thus, for instance, nothing is used from abstract algebra. This had to be compensated by using more results from topology and complex variables than is customary in introductory algebraic geometry texts. Still, aside from some harder results used in occasional examples, only basic notions and theorems are required.

Throughout the history of algebraic geometry the emphasis constantly shifted between the algebraic and the geometric sides. The first major step was a detailed study of algebraic curves by Riemann. He approached the subject from geometry and analysis, and gave a quite satisfactory structure theory. Subsequently the German school, headed by Max Noether, introduced algebra to the subject and problems arising from algebraic geometry substantially influenced the development of commutative algebra, especially the works of Emmy Noether and Krull.

During the same period the Italian school of Castelnuovo, Enriques, and Severi investigated the geometry of algebraic surfaces and achieved a satisfactory structure theory. Their work, however, lacked the Hilbertian rigor, and

Received by the editors December 3, 1986.

1980 Mathematics Subject Classification (1985 Revision). Primary 14-02, 14E30, 14E35, 32J25, 14J10, 14J15, 14E05, 14J30. 
therefore started to fall into disrepute. When the students tried their hands at studying algebraic threefolds, their conclusions were frequently false and none of the proofs of the deep results stood up to even their own standards.

Several attempts were made to place algebraic geometry on solid foundations. After substantial achievements of van der Waerden, this was accomplished by Weil and Zariski through a systematic use of commutative algebra. A good indication of this style is the two-volume treatise, Commutative algebra, by Zariski and Samuel, which grew out of an attempt to write an introductory chapter to a planned algebraic geometry book.

The approach via commutative algebra was further developed by Nagata, and it culminated in the unfinished magnum opus, Eléments de géométrie algébrique by Grothendieck. In his treatment, commutative algebra and algebraic number theory became special cases of algebraic geometry. A spectacular success of this point of view is Faltings' proof of the Mordell conjecture.

By the end of the sixties, the foundational work was mostly done and attention turned toward more classical problems. First the theory of curves and surfaces was redone and completed. A structure theory of threefolds seemed, however, intractable.

In 1972 Iitaka proposed some bold and interesting conjectures concerning higher-dimensional varieties. Along that path Ueno produced in 1977 the first structure theorem about threefolds. It was clear, however, that the scope of their approach was limited. One major stumbling block was the lack of a good analog of the so-called minimal models of surfaces.

The major breakthrough came in 1980. Mori introduced several new ideas and accomplished the first major step toward proving the existence of minimal models. At the same time Reid defined and investigated minimal models (assuming their existence) and pointed out various ways of using them. Since then algebraic threefolds have been investigated intensively and recently these efforts resulted in the proof of several deep structure theorems.

The aim of this article is to give an accessible outline of these results.

Chapters 2-5 provide a short introduction to algebraic geometry. Chapter 6 discusses the topology of algebraic varieties from the point of view of Mori's theory. Classically, one attempted to study a variety by studying its codimension-one subvarieties. A fundamental observation of Mori's theory is that one should investigate the one-dimensional subvarieties as well. This turns out to be related to some simple topological properties of algebraic varieties. After some further introductory material, Chapter 8 gives a general discussion of the basic strategy and aim of the structure theory. Chapter 9 outlines how most of this program can be accomplished for surfaces. Chapter 10 is devoted to Mori's seminal paper. These results are sufficient to complete the structure theory of surfaces, but they provide only the first step in general. In dimension three, Mori's program leads to the study of certain singular varieties. His results are reworked in this more general context in Chapter 11. The last remaining step, the Flip Theorem, is discussed in Chapter 12, and the last chapter is devoted to further results.

Since this article is aimed at a general readership, I saw no point in giving references to technical research papers. Instead I provide a short annotated bibliography at the end which should satisfy the needs of most readers. 
Part of this article was written while I enjoyed the hospitality of École Polytechnique. Financial support was also provided by the National Science Foundation (DMS-8503734).

A preliminary version of this article was circulated in the fall of 1986 . Several mistakes, inaccuracies and misprints were called to my attention by $\mathbf{R}$. Friedman, L. Lempert, K. Matsuki, T. Oda, M. Reid, H. Rossi, Y. Shimizu, and G. Tian. H. Clemens and S. Mori pointed out several conceptual problems in the presentation which led to substantial revisions. I am very grateful for all their attention and help.

\section{What is algebraic geometry?}

2.1. Starting PoINT. After Descartes introduced coordinates on the plane, it became clear that simple and much studied geometric objects (e.g., lines and conics) can be defined by simple polynomial equations (linear, resp. quadratic). This suggests that as a next step one could try to study curves defined by higher-degree polynomials. Already Newton studied plane cubics in depth. Two problems, however, tended to make results cumbersome. The first one is the missing infinity. Two different lines mostly intersect in a point, but sometimes they are parallel. It turns out to be very convenient to claim that they intersect at infinity. This leads to the introduction of the projective plane $\mathbf{R P}^{2}$. The other problem is apparent already with one-variable polynomials: the roots of a decent-looking polynomial can be lurking in the complex plane far away from the reals. Even when the real picture seems good, the explanation of certain phenomena might be seen only by studying them over C: For example, why does the Taylor series of $\left(1+x^{2}\right)^{-1}$ refuse to converge everywhere? Therefore we replace $\mathbf{R}$ by $\mathbf{C}$ and get $\mathbf{C} \mathbf{P}^{2}$. There is no reason to stop with dimension 2 and so we introduce:

2.2. Definition of $\mathbf{C P}^{n}$. As a point set this consists of $(n+1)$-tuples $\left(x_{0}: \ldots: x_{n}\right)$ such that $\left(x_{0}: \ldots: x_{n}\right)$ and $\left(\lambda x_{0}: \ldots: \lambda x_{n}\right)$ are identified for $0 \neq \lambda \in \mathbf{C}$. We exclude $(0: \ldots: 0)$.

Let $U_{i}=\left\{\left(x_{0}: \ldots: x_{n}\right) \in \mathbf{C P}^{n} \mid x_{i} \neq 0\right\}$. The map $\phi_{i}: \mathbf{C}^{n} \rightarrow \mathbf{C P}^{n}$ given by $\left(y_{1}, \ldots, y_{n}\right) \rightarrow\left(y_{1}: \ldots: y_{i}: 1: y_{i+1}: \ldots: y_{n}\right)$ maps $\mathbf{C}^{n}$ onto $U_{i}$ in a $1: 1$ way. Since $(0: \ldots: 0)$ is not in $\mathbf{C P}^{n}$, the $U_{i}$ cover it, and using the usual topology of $\mathbf{C}^{n}$, the maps $\phi_{i}$ put a topology on $\mathbf{C P}^{n}$. It is easy to see that $\mathbf{C P}^{n}$ becomes a real $2 n$-dimensional compact manifold. Despite this we shall count complex dimensions and say that $\mathbf{C}^{n}$ and $\mathbf{C P}^{n}$ are $n$-dimensional. The usual notion of dimension will be referred to as real or topological dimension. This is twice the complex dimension.

2.3. Definition of Algebraic VARIETIES. We want to consider subsets of $\mathbf{C P}^{n}$ that can be defined by polynomial equations. An arbitrary polynomial $f\left(x_{0}, \ldots, x_{n}\right)$ would not do, because $f\left(x_{0}, \ldots, x_{n}\right)$ and $f\left(\lambda x_{0}, \ldots, \lambda x_{n}\right)$ need not be both zero. However, if $f$ is homogeneous of degree $m$, then $f\left(\lambda x_{0}, \ldots, \lambda x_{n}\right)=\lambda^{m} f\left(x_{0}, \ldots, x_{n}\right)$ and the set of zeroes of $f$ in $\mathbf{C P}^{n}$ makes sense. Therefore we say that a subset $V \subset \mathbf{C P}^{n}$ is a projective algebraic variety if there are homogeneous polynomials $f_{1}, \ldots, f_{k}$ such that $V=\{(\mathbf{x}) \in$ $\left.\mathbf{C P}^{n} \mid f_{1}(\mathbf{x})=\cdots=f_{k}(\mathbf{x})=0\right\}$. One can easily see that the intersection or union of two projective algebraic varieties is again projective algebraic. 
The difference of two projective varieties is not projective usually; these are called quasiprojective varieties.

Of special interest are the varieties of the form $V \cap U_{i}$ where $V$ is projective. With the notation of 2.2 , if $V$ is defined by equations $f_{1}(\mathbf{x})=\cdots=f_{k}(\mathbf{x})=0$ then $\phi_{i}^{-1}(V) \subset \mathbf{C}^{n}$ is defined by equations $f_{j}\left(y_{1}, \ldots, y_{i}, 1, y_{i+1}, \ldots, y_{n}\right)=0$, $j=1, \ldots, k$. Such subsets of $\mathbf{C}^{n}$ are called affine varieties. The projective variety $V$ can be thought of as being patched together from the affine varieties $V \cap U_{i}$.

2.4. Definition. Algebraic geometry is the branch of mathematics that studies algebraic varieties.

2.5. Conceptual PROBlems. It is not clear that it is reasonable to study algebraic varieties. The forced union of algebra and geometry might be a bad match. There are three basic questions that have to be answered satisfactorily in order to believe that we are after something interesting.

(i) What is the relationship between the variety and its equations? Completely different equations might define the same variety!

(ii) Why is the definition so global? Maybe we should work locally on $\mathbf{C P}^{n}$ !

(iii) Are we doing intrinsic or extrinsic geometry? Why do we rely so much on the ambient space $\mathbf{C P}^{n}$ ?

The rest of this chapter will be devoted to answering these questions.

\section{Answer to Problem (i)}

2.6. It is easier to study the affine case, i.e., varieties in $\mathbf{C}^{n}$. As an illustration we consider the case of plane curves. Let $H=\left\{(x, y) \in \mathbf{C}^{2} \mid h(x, y)=0\right\}$, $F=\left\{(x, y) \in \mathbf{C}^{2} \mid f(x, y)=0\right\}$. We are interested in deciding when $H \subset F$.

Let $h=\Pi h_{i}$ be its decomposition into irreducible factors and $H_{i}=\{(x, y)$ $\left.\in \mathbf{C}^{2} \mid h_{i}(x, y)=0\right\}$. Clearly $H=\bigcup H_{i}$ and thus we have to study the conditions $H_{i} \subset F$. Let $g$ be one of the $h_{i}$ 's, and $G$ the corresponding curve.

2.7. Proposition. Let $g, f \in \mathbf{C}[x, y]$ and assume that $g$ is irreducible. Let $G$, $F$ be the corresponding curves. Then $G \subset F$ iff $g$ divides $f$.

Proof. In general this requires a little field theory, but the case $g=x^{2}+y^{2}$ -1 gives a good illustration. First suppose that $f$ has rational coefficients and pick any transcendental number, say $e$. Pick $e^{\prime}$ such that $g\left(e, e^{\prime}\right)=0$, i.e., $e^{\prime}$ $=\sqrt{1-e^{2}}$. I claim that $f\left(e, e^{\prime}\right)=0$ iff $g$ divides $f$. We divide $f$ by $g$ to get

$$
f(x, y)=\left(y^{2}+x^{2}-1\right) v(x, y)+y p(x)+q(x) \text {. }
$$

If $f\left(e, e^{\prime}\right)=0$ then $e^{\prime} p(e)+q(e)=0$. Substituting $e^{\prime}=\sqrt{1-e^{2}}$ and getting rid of the square root gives

$$
\left(1-e^{2}\right) p^{2}(e)=q^{2}(e) .
$$

Since $f$ has rational coefficients so does $p$ and $q$, hence the above gives a polynomial identity for $e$, which is impossible unless the two sides are equal:

$$
\left(1-x^{2}\right) p^{2}(x)=q^{2}(x) \text {. }
$$

This is impossible since $1-x^{2}$ is not a square, so $p$ and $q$ are identically zero. That is what we wanted. 
If $f$ has nonrational coefficients, then instead of $e$ we have to pick some $e_{0} \in \mathrm{C}$ that cannot satisfy any polynomial equation with the coefficients of $f$. The existence of $e_{0}$ follows easily from the fact that $\mathbf{C}$ is uncountable. This proves 2.7 .

Going back to $h$ and $f$ we see that $h_{i}$ divides $f$ for every $i$, so $h$ divides $f^{k}$. This implies that $H \subset F$ iff $h$ divides some power of $f$.

In general one has the following fundamental result:

2.8. HilberT's Nullstellensatz. Let $V \subset \mathbf{C}^{n}$ be defined by the polynomials $g_{1}(\mathbf{x})=\cdots=g_{k}(\mathbf{x})=0$, and let $f$ be an arbitrary polynomial in $n$ variables. Then $f$ vanishes identically on $V$ iff there exists a positive natural number $m$ and polynomials $h_{i}$ such that $f^{m}=h_{1} g_{1}+\cdots+h_{k} g_{k}$.

In short, a polynomial vanishes on an algebraic variety only if it has a clear algebraic reason to do so. Similarly the equality or containment of two varieties is equivalent to the obvious algebraic conditions.

\section{Answer to Problem (ii)}

2.9. In order to get a more local definition one could consider subsets of $\mathbf{C P}^{n}$ that are locally the zero sets of polynomials. This class is however too big; any open subset of $\mathbf{C P}^{n}$ is in it. It is reasonable to restrict to closed subsets. One can further attempt to make it more local by considering power series instead of polynomials. This leads to the following definition.

2.10. Definition. A subset $V \subset \mathbf{C P}^{n}$ is called an analytic subvariety if each point $p$ of $V$ has a neighborhood $B_{p}$ such that $V \cap B_{p}=\left\{(\mathbf{x}) \in B_{p} \mid f_{1}(\mathbf{x})=\right.$ $\left.\cdots=f_{k}(\mathbf{x})=0\right\}$ for some analytic functions $f_{i}$ defined on $B_{p}$. (If $B_{p}$ is small, then it is contained in some $U_{i} \simeq \mathbf{C}^{n}$, so it makes sense to talk about analytic functions on $B_{p}$.)

It is clear that every algebraic variety is an analytic variety. It is quite a miracle that the converse is also true:

2.11. TheOREM OF CHOw. Let $V \subset \mathbf{C P}^{n}$ be a closed analytic subvariety. Then $V$ is algebraic, i.e., can be globally defined by polynomials.

Proof. We shall give a proof for $V \subset \mathbf{C P}^{2}$; the general case can be treated similarly. First we need some results about the local structure of analytic subvarieties of $\mathbf{C}^{2}$. The crux is the following:

2.12. WeIERSTRASS PREPARATION THEOREM. Let $f(x, y)$ be a holomorphic function around the origin. Assume that $f(0,0)=0$ and $f(0, y) \not \equiv 0$. Then there are power series $b_{1}(x), \ldots, b_{k}(x)$ and $u(x, y)$ such that $u(0,0) \neq 0$ and

$$
f(x, y)=\left(y^{k}+b_{1}(x) y^{k-1}+\cdots+b_{k}(x)\right) u(x, y) \text {. }
$$

Proof. Since $f(0, y)$ is not identically zero, there is a small $\varepsilon$ such that $f(0, y) \neq 0$ for $|y|=\varepsilon$, and hence for some $\delta>0$ we have $f(x, y) \neq 0$ if $|y|=\varepsilon,|x|<\delta$. For fixed $x$ let $r_{1}(x), \ldots, r_{k}(x)$ be the roots of $f(x, \cdot)=0$ inside the disc of radius $\varepsilon$ (a priori $k$ might depend on $x$ ). By the residue theorem

$$
r_{1}^{m}(x)+\cdots+r_{k}^{m}(x)=\frac{1}{2 \pi i} \int_{|y|=\varepsilon} \frac{y^{m} \partial f / \partial y}{f} d y
$$


If $|x|<\delta$ then the right-hand side gives a holomorphic function of $x$. In particular for $m=0$, we get that $k$ is independent of $x$. Let $\sigma_{1}(x), \ldots, \sigma_{k}(x)$ be the elementary symmetric polynomials in the $r_{i}(x)$ 's. These are polynomials in the sums of powers, hence holomorphic in $x$.

Now by construction $f(x, y)$ and $y^{k}-\sigma_{1}(x) y^{k-1}+\cdots+(-1)^{k} \sigma_{k}(x)$ vanish on exactly the same set, hence their quotient $u(x, y)$ is holomorphic and nonzero. This completes the proof. $\mathrm{C}^{2}$ :

As a consequence we can describe the local structure of analytic subsets of

2.13. Proposition. Let $V$ be an analytic subvariety in the neighborhood of the origin of $\mathbf{C}^{2}$. Then $V=U \cup W$, where $U$ is a finite set and $W=\{(x, y) \in$ $\left.\mathrm{C}^{2} \mid g(x, y)=0\right\}$ is the zero set of one power series.

Proof. Let $V$ be defined by $f_{1}=\cdots=f_{m}=0$. By a suitable change of coordinates one can assume that none of the $f_{i}$ 's is identically zero on the $y$-axis. By 2.12 each $f_{i}$ can be written as

$$
f_{i}(x, y)=g_{i}(x, y) u_{i}(x, y),
$$

where $g_{i}$ is a polynomial in $y$ whose coefficients are power series in $x$. Since $u_{i}(0,0) \neq 0$, we have $V=\left\{(x, y) \in \mathbf{C}^{2} \mid g_{1}=\cdots=g_{m}=0\right\}$, at least near the origin.

Now let $g_{12}$ be the g.c.d. of $g_{1}$ and $g_{2}$ (as polynomials in $y$ ), and let $g_{i}=h_{i} \cdot g_{12}(i=1,2)$. Clearly we can write

$$
\left\{(x, y) \mid g_{1}=g_{2}=0\right\}=\left\{(x, y) \mid g_{12}=0\right\} \cup\left\{(x, y) \mid h_{1}=h_{2}=0\right\} .
$$

I claim that the last set is finite. Indeed for any fixed $x$ consider the resultant of $h_{1}(x, y)$ and $h_{2}(x, y)$. This is a certain polynomial in the coefficients of the $h_{i}$ 's, hence it varies holomorphically with $x$. Since $h_{1}$ and $h_{2}$ are coprime, the resultant is not identically zero. Thus it has only finitely many zeroes near the origin. For each of these zeroes $h_{1}$ and $h_{2}$ have only finitely many common roots. These are all the solutions of $h_{1}=h_{2}=0$.

Now let $g_{123}$ be the g.c.d. of $g_{12}$ and $g_{3}, \ldots$ Eventually we get that one can take $g$ to be the g.c.d. of $g_{1}, \ldots, g_{m}$. This completes the proof.

2.14. It is of interest to see to what extent $g$ is unique. Let $g=h_{1} \cdots h_{s}$ be its decomposition into irreducible factors. If $h_{i}(0,0) \neq 0$, then since $h_{i}$ has no zeroes near the origin, it can be discarded. It is also clear that a factor should occur only once. This shows that one can choose a defining equation $\bar{g}$ such that for the irreducible decomposition $\bar{g}=\bar{h}_{1} \cdots \bar{h}_{t}$ there are no multiple factors and $\bar{h}_{i}(0,0)=0$. The previous proof shows that this $\bar{g}$ is unique (although of course it depends on the choice of the local coordinates).

Next we derive a more global version of 2.13:

2.15. Proposition. Let $D \subset \mathbf{C}$ be a connected open subset and $V \subset D \times \mathbf{C}$ be a closed analytic subset without isolated points. Assume that the projection $p$ : $V \rightarrow D$ is proper. Then there is a unique power series without multiple factors

$$
g=y^{k}+a_{1}(x) y^{k-1}+\cdots+a_{k}(x)
$$

such that $V=\{(x, y) \in D \times \mathbf{C} \mid g(x, y)=0\}$. 
Proof. For each $v \in V$ we pick the unique local defining equation $\bar{g}_{v}$ as in 2.14. For each $x \in D$ let $\left(x, b_{1}(x)\right), \ldots,\left(x, b_{k}(x)\right)$ be the points of $V$ above $x$ (with multiplicities dictated by the $\bar{g}_{v}$ 's). As in 2.12 we see that $k$ is locally constant, hence constant since $D$ is connected. Similarly we see that the elementary symmetric polynomials of the $b_{i}$ 's are locally holomorphic, giving $g(x, y)$. Uniqueness is again clear.

2.16. Now we are ready to prove the theorem of Chow.

By 2.13 one can write $V=U \cup W$, where $U$ is locally finite (therefore finite) and $W$ is locally defined by one equation. All we have to do is to show that $W$ is algebraic.

On $\mathbf{C P}^{2}$ we choose coordinates $\left(z_{0}: z_{1}: z_{2}\right)$, and we can assume that $(0: 1: 0)$ $\notin W$. Our strategy is the following. Let $p: \mathbf{C P}^{2}-(0: 1: 0) \rightarrow \mathbf{C P}^{1}$ be the projection $\left(z_{0}: z_{1}: z_{2}\right) \rightarrow\left(z_{0}: z_{2}\right)$.

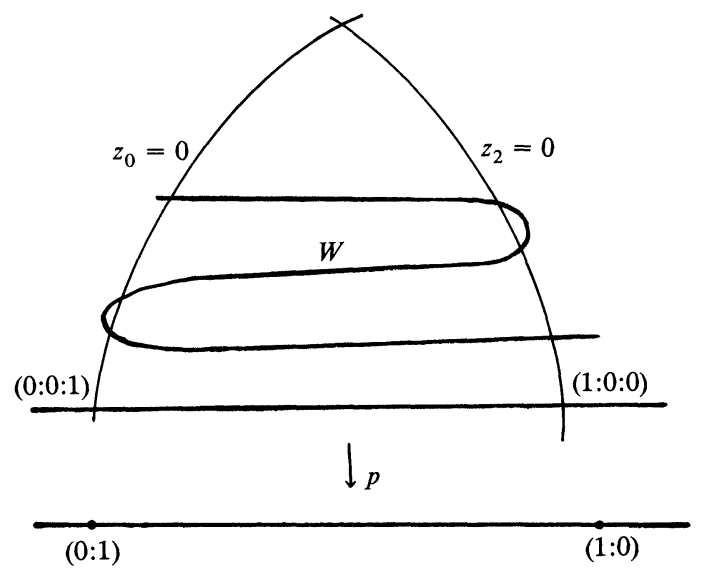

Figure 1

On $U_{2}=\mathbf{C P}^{2}-\left(z_{2}=0\right)$ we have the coordinate chart $y=z_{1} / z_{2}, x=$ $z_{0} / z_{2}$, and $p$ corresponds to the projection $(x, y) \rightarrow x$. Since $W$ is compact, $p$ is proper on $W$, and therefore on $U_{2} \cap W$. Hence by 2.15 we get that $U_{2} \cap W$ can be defined by a unique equation $y^{k}+a_{1}(x) y^{k-1}+\cdots=0$, where the $a_{i}$ are holomorphic in $x$. Our aim is to prove that the $a_{i}$ are in fact polynomials in $x$. This follows once we know that they don't grow too fast as $x \rightarrow \infty$.

To see this we look at the other chart $U_{0}=\mathbf{C P}^{2}-\left(z_{0}=0\right)$ with coordinates $u=z_{1} / z_{0}, v=z_{2} / z_{0}$. Here $p$ is the projection $(u, v) \rightarrow v$. Therefore $U_{0} \cap W$ can be defined by an equation of the form $u^{m}+b_{1}(v) u^{m-1}+\cdots=0$.

On $U_{02}=U_{0} \cap U_{2}$ we have two equations of $W \cap U_{02}$ : one coming from $U_{0}$, the other form $U_{2}$. By uniqueness these two equations agree, and this allows us to understand the behavior of $a_{i}$ as $x \rightarrow \infty$.

Using $u=y / x, \quad v=1 / x$, the second equation becomes $(y / x)^{m}+$ $b_{1}(1 / x)(y / x)^{m-1}+\cdots=0$. This is not of the form we expect, but we can multiply through by $x^{m}$ (which is nowhere zero on $U_{02}$ ) to get $y^{m}+$ $x b_{1}(1 / x) y^{m-1}+\cdots=0$. This must be the same as our original equation 
$y^{k}+a_{1}(x) y^{k-1}+\cdots=0$. Hence $k=m$ and

$$
a_{i}(x)=x^{i} b_{i}(1 / x) \text {. }
$$

$b_{i}(1 / x)$ is holomorphic near $\infty$, therefore $a_{i}$ grows at most as $x^{i}$; hence $a_{i}$ is a polynomial of degree at most $i$.

Therefore the homogeneous polynomial defining $W$ is given by

$$
g\left(z_{0}, z_{1}, z_{2}\right)=z_{1}^{m}+z_{2} a_{1}\left(z_{0} / z_{2}\right) z_{1}^{m-1}+\cdots+z_{2}^{m} a_{m}\left(z_{0} / z_{2}\right) .
$$

This completes the proof.

We proved in fact a bit more:

2.17. Corollary. Let $W \subset \mathbf{C P}^{2}$ be a closed analytic subvariety without isolated points. Then $W$ can be defined by one polynomial equation.

The preceding results can be used to obtain some further nice connections between geometry and algebra.

2.18. Definition. An algebraic variety is called irreducible if it cannot be written as the union of two proper closed subvarieties. It is quite clear that $\mathbf{C P}^{n}$ is irreducible.

2.19. Proposition. A plane curve $G \subset \mathbf{C P}^{2}$ is irreducible iff it can be defined by an irreducible equation.

Proof. Let $g=g_{0} \cdots g_{i}$ be the defining equation with no multiple factors. $G=\left(g_{1}=0\right) \cup \cdots \cup\left(g_{i}=0\right)$ so if $i \geqslant 2$ then $G$ is reducible. Conversely let $G=G_{1} \cup G_{2}$. We can clearly assume that $G_{i}$ has no isolated points. By 2.17 it is defined by an equation $g_{i}=0$. By $2.7 g_{i} \mid g$; hence $g$ is reducible.

2.20. Proposition. An irreducible algebraic variety is connected.

Proof. Let $V_{i}$ be a connected component of $V . V_{i}$ is clearly a closed analytic subvariety of $\mathbf{C P}^{n}$, hence algebraic by 2.11 . This contradicts the irreducibility.

There are two other simple but useful results concerning the topology of algebraic varieties.

2.21. Proposition. Let $U$ be an irreducible algebraic variety and let $V \neq U$ be a closed subvariety. Then $U-V$ is dense in $U$.

Proof. Assume that $U$ is smooth. Then locally $U$ looks like $\mathbf{C}^{n}$ and $V$ is defined by equations $f_{1}=\cdots=f_{k}=0 . \mathbf{C}^{n}-\left(f_{1}=0\right)$ is smaller than $\mathbf{C}^{n}-$ $\left(f_{1}=\cdots=f_{k}=0\right)$ and it is clearly dense in $\mathbf{C}^{n}$. The general case is considerably harder.

2.22. THEOREM. Let $U$ be an algebraic variety and let $V$ be a closed subvariety. If $W \subset U-V$ is a closed subvariety, then $\bar{W}$, the closure of $W$ in $U$, is again an algebraic variety.

Proof. We do the simple case $U=\mathbf{C P}^{n}, V=\left(x_{0}=0\right)$. The general case is very similar. We may assume that $W$ is irreducible. $U-V \cong \mathbf{C}^{n}$ and $W$ is defined by equations $f_{j}\left(x_{1} / x_{0}, \ldots, x_{n} / x_{0}\right)=0$. If $d$ is large, then $x_{0}^{d} f_{j}$ are polynomials in $x_{0}, \ldots, x_{n}$ and they are homogeneous. They define a closed subvariety $W^{\prime} \subset \mathbf{C P}^{n}$ such that $W^{\prime} \cap \mathbf{C}^{n}=W$. In general $W^{\prime}$ is reducible, 
but it has an irreducible component $W^{\prime \prime}$ such that $W \subset W^{\prime \prime}$. By 2.21, $W=W^{\prime \prime}-\left(x_{0}=0\right)$ is dense in $W^{\prime \prime}$. Hence $\bar{W}=W^{\prime \prime}$.

It is important to note that 2.22 is notoriously false for analytic varieties.

One can try to define analytic varieties that do not a priori sit in $\mathbf{C P}^{n}$, and these indeed can be nonalgebraic (see Example 4.8). For simplicity we define the manifold case only.

2.23. Definition. A complex manifold of dimension $n$ is a topological manifold $M$ of real dimension $2 n$ which is covered by coordinate charts $\cup U_{i}=M$, and for each chart we fix an injective homeomorphism $\phi_{i}: U_{i} \rightarrow \mathrm{C}^{n}$ onto some open subset. A function $f$ on $M$ is said to be holomorphic if $f \circ \phi_{i}^{-1}$ is holomorphic for every $i$. For this to make good sense we would like that on $U_{i} \cap U_{j}$ the notion of holomorphy is the same viewed from $U_{i}$ or from $U_{j}$. Therefore we have to impose the assumption that the $\phi_{i} \phi_{j}^{-1}$ are all holomorphic.

2.24. EXAmple. $\mathbf{C P}^{n}$ is a complex manifold with the charts given in its definition 2.2.

2.25. ExAmple. Let $f(\mathbf{x})$ be holomorphic on $\mathbf{C}^{n}$. Let $F=(f(\mathbf{x})=0)$. Assume that for every $(\mathbf{x}) \in F$ at least one of the partial derivatives $\partial f / \partial x_{i}(\mathbf{x})$ is not zero. If, say, $\partial f / \partial x_{1}(\mathbf{x}) \neq 0$, then by the implicit function theorem the projection $F \rightarrow \mathbf{C}^{n-1}:\left(x_{1}, \ldots, x_{n}\right) \rightarrow\left(x_{2}, \ldots, x_{n}\right)$ is locally a homeomorphism near (x). This gives charts on $F$ and one can check that this makes $F$ into a complex manifold.

This guides us in defining the notion of a smooth point for algebraic varieties. Since $\mathbf{C P}^{n}$ is covered by copies of $\mathbf{C}^{n}$, it is sufficient to define the notion for affine varieties.

2.26. Definition. Let $V \subset \mathbf{C}^{n}$ be an algebraic variety and let $v \in V$. We say that $V$ is smooth or nonsingular of dimension $k$ at $v$ if there is a $\mathbf{C}^{k} \subset \mathbf{C}^{n}$ such that, for a suitable projection $p: \mathbf{C}^{n} \rightarrow \mathbf{C}^{k}$, the map $p: V \rightarrow \mathbf{C}^{k}$ is a homeomorphism near $v$. The same will hold then for most choices of $\mathbf{C}^{k}$ and p. It is also clear that being smooth is an open condition. Points which are not smooth are called singular; they form a closed subset $\operatorname{Sing} V \subset V$. This subset turns out to be algebraic. It is again quite clear that $V-\operatorname{Sing} V$ becomes a complex manifold with the obvious charts.

The following result relates the number of defining equations and the dimension of an algebraic variety.

2.27. TheOREM. Let $V \subset \mathbf{C}^{n}$ be an algebraic variety and let $f_{1}, \ldots, f_{k}$ be polynomials. Let $W$ be an irreducible component of $V \cap\left(f_{1}=\cdots=f_{k}=0\right)$. Then

$$
\operatorname{dim} W \geqslant \operatorname{dim} V-k
$$

Proof. It is clearly sufficient to prove this for $k=1$. For simplicity let us assume that $V$ is smooth so that we consider the case when $f$ is holomorphic on the unit ball of $\mathbf{C}^{m}(m=\operatorname{dim} V)$. If $(f=0)$ is not empty and not everything, then by a suitable choice of coordinates $f(0)=0$ and $f$ is not identically zero on the $z_{1}$-axis. Thus for a suitable choice of $\varepsilon$ and $\delta, f$ does not 
vanish for $\left|z_{1}\right|=\varepsilon,\left|z_{2}\right| \leqslant \delta, \ldots,\left|z_{m}\right| \leqslant \delta$. The integral

$$
N\left(z_{2}, \ldots, z_{m}\right)=\frac{1}{2 \pi i} \int_{\left|z_{1}\right|=\varepsilon} \frac{\partial f / \partial z_{1}}{f} d z_{1}
$$

counts the number of zeroes of $f\left(*, z_{2}, \ldots, z_{m}\right)$ on the disc $\left|z_{1}\right|<\varepsilon$. It is continuous for $\left|z_{2}\right| \leqslant \delta, \ldots,\left|z_{m}\right| \leqslant \delta$, hence constant. By assumption $N(0, \ldots, 0)>0$; therefore the projection of $(f=0)$ onto the $\left(z_{2}, \ldots, z_{m}\right)$ plane is surjective near the origin. Thus $\operatorname{dim}(f=0)=m-1$.

2.28. REMARK. (i) If $V$ is smooth and $W \subset V$ is an irreducible subvariety such that $\operatorname{dim} W=\operatorname{dim} V-1$, then locally $W$ can be defined by a single equation. This is the higher-dimensional analog of 2.13 .

(ii) The previous remark does not hold for $V$ singular (see 4.4).

(iii) If $V$ is smooth and $\operatorname{dim} W=\operatorname{dim} V-2$, then $W$ might not be definable by two equations (see 4.3).

\section{Answer to Problem (iii)}

2.29. The answer to this is not a nice theorem but rather a conceptual understanding. First of all, it is quite possible to build up algebraic geometry without any reference to $\mathbf{C P}^{n}$ or $\mathbf{C}^{n}$. The important fact is however that the intrinsic and extrinsic geometry of a variety are nearly inextricable. To start with first note that $\mathbf{C P}^{n}$ is very rigid (a proof will be given in 7.18):

\subsection{Proposition. Aut $\mathbf{C P}^{n} \simeq \operatorname{PGL}(n+1, \mathrm{C})$.}

Here Aut $\mathbf{C P}^{n}$ is the group of 1:1 self-maps of $\mathbf{C P}^{n}$ that are given by polynomials, or, by a version of Chow's theorem, those that are locally given by power series. $\mathrm{GL}(n+1, \mathrm{C})$ operates naturally on $(n+1)$-tuples, and this gives an action on $\mathbf{C P}^{n}$. Scalar matrices operate trivially, therefore $\operatorname{PGL}(n+1)$ operates on $\mathbf{C P}^{n}$.

The crux of the matter is that there are no other automorphisms. Therefore, for instance, collineation is an abstract property of point sets in $\mathbf{C P}^{n}$ !

More complicated algebraic varieties usually have no automorphisms at all.

2.31. An even more remarkable fact is that frequently algebraic varieties fit into some $\mathbf{C P}^{n}$ in a unique way. For instance, if $G \subset \mathbf{C P}^{2}$ is an irreducible curve of degree at least 4 , then there is only one way this curve can fit into $\mathbf{C P}^{2}$. So manifestly extrinsic properties of points of $G$ such as being an inflection point or such as two points having a common tangent line turn out to be intrinsic properties after all.

The real importance of this principle is that it leads to a "linearization process" of algebraic varieties. I will explain it for algebraic curves only. The reader not familiar with curves should read $\$ 3$ first.

2.32. CHOW COORDINATES OF CURVES. Let $C$ be a smooth projective curve of genus $g \geqslant 2$. We distinguish two cases.

(i) $C$ admits a 2:1 map onto $\mathbf{C P}^{1}$. Such curves are called hyperelliptic. It turns out that this map is essentially unique. There are exactly $2 g+2$ points in $\mathbf{C P}^{1}$ above which the map is $1: 1$. These points determine $C$. Thus we have a correspondence

$$
\left\{\begin{array}{c}
\text { hyperelliptic curves } \\
\text { of genus } g
\end{array}\right\} \leftrightarrow\left\{\begin{array}{c}
\text { sets of } 2 g+2 \\
\text { points in } \mathbf{C P}^{1}
\end{array}\right\} / \text { Aut } \mathbf{C P}^{1} \text {. }
$$


$2 g+2$ points can be viewed as the zero set of a degree $2 g+2$ homogeneous polynomial $f(x, y)$. Thus we can write

$$
\left\{\begin{array}{c}
\text { hyperelliptic curves } \\
\text { of genus } g
\end{array}\right\} \leftrightarrow\left\{\begin{array}{c}
\text { degree } 2 g+2 \text { homogeneous polynomials } \\
\text { without multiple roots }
\end{array}\right\} / \mathrm{GL}(2, \mathbf{C})
$$

where $\operatorname{GL}(2, \mathrm{C})$ acts via

$$
\left(\begin{array}{ll}
a & b \\
c & d
\end{array}\right) f(x, y)=f(a x+b y, c x+d y)
$$

Thus hyperelliptic curves can be understood via certain simple "linear objects," namely polynomials.

(ii) $C$ is not hyperelliptic. Then one can prove that there is an essentially unique embedding $C \subset \mathbf{C} \mathbf{P}^{g-1}$ such that $C$ is not contained in any hyperplane and that a general hyperplane intersects $C$ in $2 g+2$ points. Using this embedding we will associate a "linear object" to $C$. Let $V=\left\{\sum a_{i} x_{i}\right\} \cong \mathbf{C}^{g}$ be the space of homogeneous linear polynomials on $\mathbf{C P}^{g-1}$. Let $\mathrm{Ch}(C) \subset V \times V$ be the set of pairs $\left(l_{1}, l_{2}\right) \in V \times V$ such that $\left(l_{1}=0\right) \cap\left(l_{2}=0\right) \cap C \neq \varnothing$.

If we fix $l_{2}$, then $\left(l_{2}=0\right) \cap C$ is a finite set of points. Thus $\left(l_{1}=0\right) \cap$ $\left(l_{2}=0\right) \cap C \neq \varnothing$ imposes one condition on $l_{1}$. Therefore $\operatorname{Ch}(C)$ is a codimension one subset in $V \times V$ and as such it can be defined by a single equation $\operatorname{ch}(C)\left(a_{1}, \ldots, a_{g}, a_{1}^{\prime}, \ldots, a_{g}^{\prime}\right)$. The above considerations show that $C$ can be reconstructed from $\operatorname{ch}(C)$. It is easy, to see that $\operatorname{ch}(C)$ is homogeneous of degree $2 g+2$ in either set of $g$-variables. Under the action of $\mathrm{GL}(g, \mathbf{C})$ on $\mathbf{C P}^{g-1}$, it is transformed by

$$
\left(b_{i j}\right) \operatorname{ch}(C)\left(a_{k}, a_{k}^{\prime}\right)=\operatorname{ch}(C)\left(\sum b_{k j} a_{j}, \Sigma b_{k j} a_{j}^{\prime}\right) .
$$

Thus we have an injection

$$
\left\{\begin{array}{c}
\text { nonhyperelliptic } \\
\text { curves of genus } g
\end{array}\right\} \rightarrow\left\{\begin{array}{c}
\text { bihomogeneous polynomials of degree } \\
(2 g+2,2 g+2) \text { in } 2 g \text { variables }
\end{array}\right\} / \mathrm{GL}(g, \mathbf{C}) \text {. }
$$

This is not as nice as before since the image is very hard to describe. Still, it provides a good conceptual way of imagining all algebraic curves together and it can be developed into a very powerful method of investigating algebraic curves.

2.33. This rigidity of maps provides a very strong tool to study algebraic varieties, but we also pay a high price for it. First of all, as we shall see, it is hard to find interesting maps. Then if we have a map it might be very hard to improve it. Standard perturbation methods of differential topology (e.g., transversality lemmas, nearby Morse functions) will not work because there will be no perturbations.

Therefore one is forced to study degenerate situations in great detail. Methods to handle such problems form the technical core of algebraic geometry, and are frequently very hard. In this survey I will gently ignore such problems, concentrating instead on the geometrically clear part of the arguments.

3. A little about curves. The simplest algebraic varieties are algebraic curves. They are very similar to one-dimensional complex manifolds. Since these have real dimension two, they are usually called Riemann surfaces. We now turn to 
their study partly to have some example at hand, partly to explain certain facts that will be needed later.

We shall concentrate on the complex manifold side of the theory. One reason for this is that the topological-analytic approach is conceptually easier. On the other hand, it turns out that for one-dimensional compact varieties the analytic and the algebraic theories are equivalent.

3.1. TOPOLOGY OF CURVES. The underlying topological space is a surface. On any chart multiplication by $\sqrt{-1}$ gives an orientation, and this is independent of the chart chosen. Compact orientable surfaces are spheres with a certain number of handles. The only invariant is the number of handles, called the genus, denoted by $g($ ).

3.2. $g=0$. The underlying topological space is a sphere, and we know one such complex manifold: $\mathbf{C P}^{1}$. This turns out to be the only one; see 3.10.

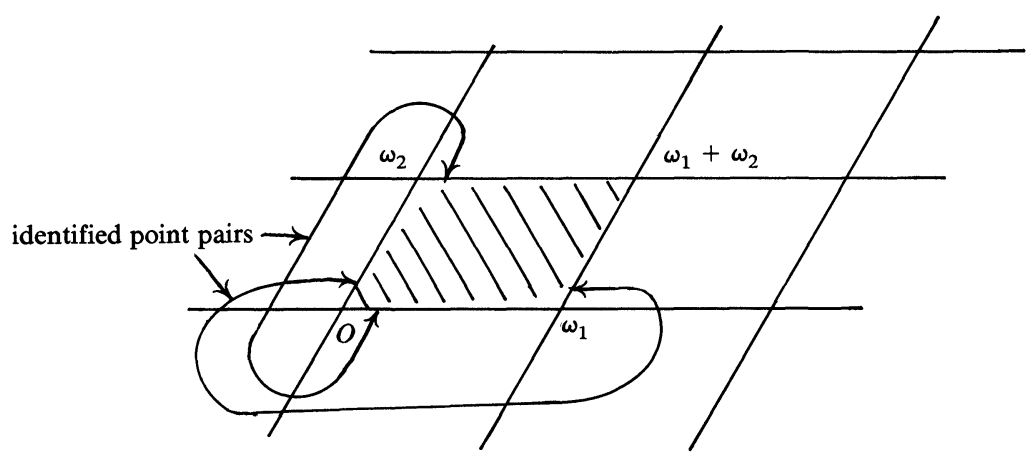

FIGURE 2

3.3. $g=1$. Books were written about this.

(i) To start with, a sphere with one handle is a torus, which is $S^{1} \times S^{1} \approx$ $\mathbf{R} / \mathbf{Z} \times \mathbf{R} / \mathbf{Z} \approx \mathbf{C} / \mathbf{Z}+\mathbf{Z}$. This is a good way to get such examples: let $\omega_{1}, \omega_{2}$ be $\mathbf{R}$-independent complex numbers, and let $L=\left\{n \omega_{1}+m \omega_{2} \mid n, m \in \mathbf{Z}\right\}$ be the lattice they generate. Identify two points of $\mathbf{C}$ if their difference is in $L$. This gives $\mathbf{C} / L$. The shaded area of the picture is a fundamental parallelogram (i.e., its translates by $L$ cover $\mathbf{C}$ ). $\mathbf{C} / L$ can be thought of as the fundamental parallelogram with opposite sides identified. Note that on $\mathbf{C}$ the addition $(x, y) \rightarrow x+y$ is holomorphic; therefore $\mathbf{C}$ is a complex Lie group. $L$ is a subgroup of $\mathbf{C}$, and this makes $\mathbf{C} / L$ into a compact complex Lie group.

(ii) When will $\left(\omega_{1}, \omega_{2}\right)$ and $\left(\omega_{1}^{\prime}, \omega_{2}^{\prime}\right)$ give the same complex manifold? Let $L$ and $L^{\prime}$ be the corresponding lattices and look at the diagram

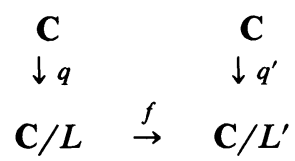

Let $f$ be a 1:1 holomorphic map. $f(0)$ need not be the origin in $\mathrm{C} / L^{\prime}$, but we can compose $f$ with a translation in $C / L^{\prime}$ to achieve this. So assume that $f(0)=0$. Let $q^{\prime}: \mathbf{C} \rightarrow \mathbf{C} / L^{\prime}$ be the universal covering map. Then $f \circ q: \mathbf{C} \rightarrow \mathbf{C} / L^{\prime}$ can be lifted to $f^{*}: \mathbf{C} \rightarrow \mathbf{C}$. We can play the same game with 
$f^{-1}$ and get $f^{-1 *}: \mathbf{C} \rightarrow \mathbf{C}$. Clearly $f^{-1 *}$ is the inverse of $f^{*}$; therefore $f^{*}$ is multiplication with some complex number $\mu . f^{*}\left(q^{-1}(0)\right)=q^{\prime-1}(0)$, hence $f^{*}(L)=L^{\prime}$; i.e., $\mu L=L^{\prime}$. Conversely if $\lambda L=L^{\prime}$ for some $\lambda$, then this gives a $1: 1 \operatorname{map} \lambda: \mathbf{C} / L \rightarrow \mathbf{C} / L^{\prime}$.

Starting with $\left(\omega_{1}, \omega_{2}\right)$ we can take $\mu=\omega_{1}^{-1}$ or $\omega_{2}^{-1}$ to get $(1, \tau)$ with $\operatorname{Im} \tau>0$. The corresponding lattice will be denoted by $L_{\tau}$, and $\mathrm{C} / L_{\tau}=E_{\tau}$.

(iii) Every $\mathrm{C} / L$ is isomorphic to some $E_{\tau} . E_{\tau}$ and $E_{\tau^{\prime}}$ are isomorphic iff

$$
\tau^{\prime}=\frac{a \tau+b}{c \tau+d} \quad \text { for some } a, b, c, d \in \mathbf{Z}, a d-b c=1 .
$$

Proof. We already saw the first part. So to see the second let $\mu: L_{\tau^{\prime}} \rightarrow L_{\tau}$ be the multiplication. Then $\mu \tau^{\prime}=a \tau+b \cdot 1$ and $\mu \cdot 1=c \tau+d \cdot 1$. The fact that $\mu \tau^{\prime}$ and $\mu \cdot 1$ generate $L_{\tau}$ gives $a d-b c= \pm 1$. One can check that the conditions on the imaginary parts force $a d-b c=+1$.

This shows a very special feature of complex manifolds: they can vary continuously. If $\tau$ is changed a little bit, we get a different manifold!

(iv) Functions on $\mathbf{C} / L$. Let $g$ be a meromorphic function on $\mathbf{C} / L$. Then $q^{*} g$ is a meromorphic function on $\mathbf{C}$ such that $q^{*} g\left(z+\omega_{1}\right)=q^{*} g\left(z+\omega_{2}\right)=$ $q^{*} g(z)$; i.e., $q^{*} g$ is doubly periodic. Conversely, such functions on $\mathbf{C}$ give functions on $\mathbf{C} / L$. These are the so-called elliptic functions. The basic one is

$$
p(z)=z^{-2}+\sum_{\omega \in L-0}\left[(z-\omega)^{-2}-\omega^{-2}\right] .
$$

With some work one can see that $p$ is meromorphic on $\mathbf{C}$, doubly periodic, and has poles exactly at $L$; these poles are double. Clearly $p^{\prime}(z)$ is again doubly periodic, and it has triple poles at $L$. I claim that $p$ and $p^{\prime}$ are related by some polynomial equation. The proof will be a bit unusual.

Consider the map

$$
\mathbf{C}-L \rightarrow \mathbf{C P}^{2} \quad z \rightarrow\left(p(z): p^{\prime}(z): 1\right) .
$$

This descends to a map $(\mathbf{C} / L)-0 \rightarrow \mathbf{C P}^{2}$. I intend to extend this over 0 . The map is the same as $z \rightarrow\left(p(z) / p^{\prime}(z): 1: 1 / p^{\prime}(z)\right)$ which is defined at $z=0$. Hence we have a map $\mathbf{C} / L \rightarrow \mathbf{C P}^{2}$. The image is a compact analytic subvariety, hence by 2.11 it satisfies some polynomial equation $f\left(x_{0}: x_{1}: x_{2}\right)=0$. This gives $f\left(p(z), p^{\prime}(z), 1\right)=0$.

In fact in this case one can write down the equation:

(v) $\left[p^{\prime}\right]^{2}=4 p^{3}+a p+b$ for some $a, b \in \mathbf{C}$. Hence the image is a cubic curve.

(vi) The last topic I want to mention is self-maps of $E_{\tau}$. If $n \in \mathbf{Z}$ then multiplication by $n$ maps $L_{\tau}$ into itself. This gives a map $n: E_{\tau} \rightarrow E_{\tau}$. This is an $n^{2}: 1$ map,

$$
n^{-1}(z)=\left\{\frac{z}{n}+\frac{i \omega_{1}+j \omega_{2}}{n}: 0 \leqslant i, j<n\right\} .
$$

3.4. $g \geqslant 2$. This case is more complicated and we shall discuss only two topics. One is the topology of maps between Riemann surfaces; the other is the analog of the Mittag-Leffler problem: find functions with prescribed poles. 
Let $C$ be a compact Riemann surface with a triangulation. Let $t, l, v$ denote the number of triangles, resp. line segments, resp. vertices in this triangulation. It is easy to see that $t-l+v=2-2 g$, where $g$ is the genus.

Now let $F: C^{\prime} \rightarrow C$ be a nonconstant holomorphic map between two Riemann surfaces. $C^{\prime}$ is covered by charts $U_{i}$, and on each chart $f$ is given by a power series $f_{i}(z)$. $f$ is not a local homeomorphism at $z \in U_{i}$ iff $f_{i}^{\prime}(z)=0$; hence such points form a discrete set, finite if $C^{\prime}$ is compact. Let $B \subset C$ be the images of these points and consider a triangulation of $C$ where $B$ is part of the set of vertices. Let $t, l, v$ be the corresponding numbers. We can pull back this triangulation to $C^{\prime}$. If $f$ is $n: 1$ outside $B$ then we get $t^{\prime}=n t, l^{\prime}=n l, p^{\prime} \leqslant n p$. Therefore

$$
2-2 g^{\prime}=t^{\prime}-l^{\prime}+v^{\prime}=n(2-2 g)-\left(n p-p^{\prime}\right) .
$$

Hence $2 g^{\prime}-2 \geqslant n(2 g-2)$. This at once gives

3.5. Proposition. Let $f: C \rightarrow C^{\prime}$ be a nonconstant map of algebraic curves. Then

(i) $g(C) \geqslant g\left(C^{\prime}\right)$. In particular if $C \cong \mathbf{C P}^{1}$ then also $C^{\prime} \cong \mathbf{C P}$.

(ii) If $g(C)=g\left(C^{\prime}\right) \geqslant 2$ then $f$ is an isomorphism.

3.6. Definition. Let $C$ be a compact Riemann surface, $P_{i} \in C$ a collection of points, and $n_{i}$ natural numbers. Let $\Gamma\left(\sum n_{i} P_{i}\right)$ be the set of all meromorphic functions on $C$ which have poles only at the $P_{i}$ 's of multiplicity at most $n_{i}$. Such functions clearly form a vector space, and the Mittag-Leffler problem asks for its dimension.

3.7. Proposition. $\operatorname{dim} \Gamma\left(\sum n_{i} P_{i}\right) \leqslant 1+\sum n_{i}$.

Proof. At each $P_{i}$ we choose a local coordinate $z_{i}$. For $f \in \Gamma\left(\sum n_{i} P_{i}\right)$ we consider the Laurent expansion of $f$ at the $P_{i}$ 's,

$$
f(z)=a_{-n_{i}} z^{-n_{i}}+\cdots+a_{-1} z^{-1}+\cdots .
$$

At $P_{i}$ there can be $n_{i}$ different negative exponents. Therefore $\operatorname{dim} \Gamma\left(\sum n_{i} P_{i}\right) \leqslant$ $\operatorname{dim} \Gamma(\varnothing)+\sum n_{i}$ where $\Gamma(\varnothing)$ is the space of holomorphic functions on $C$. By the maximum principle these are all constants; hence $\operatorname{dim} \Gamma(\varnothing)=1$.

A lower bound is much more interesting and difficult:

3.8. Theorem (RIEMANN). $\operatorname{dim} \Gamma\left(\sum n_{i} P_{i}\right) \geqslant \sum n_{i}+1-g$. If $\sum n_{i} \geqslant 2 g-1$, then we have equality.

Proof. We shall only indicate the main steps of the argument.

As a first step we shall search only for $u=\operatorname{Re} f$, which is a harmonic function. Pick a $P=P_{i}$ and a $1 \leqslant k \leqslant n_{i}$. Assume that $u$ is harmonic on $C-P$ and has a $k$ th order pole at $P$; i.e., $u$ behaves like $\operatorname{Re} z^{-k}$. By definition of harmonic, $\Delta u=\left(\partial^{2} / \partial x^{2}+\partial^{2} / \partial y^{2}\right) u \equiv 0$. The corresponding variational problem is the minimization of the Dirichlet integral

$$
\int_{C-P}\left(\frac{\partial u}{\partial x}\right)^{2}+\left(\frac{\partial u}{\partial y}\right)^{2} d x d y
$$


There are two problems with this. First, because of the pole of $u$ at $P$, the above integral is divergent. The second, more serious, is to prove that if $u$ is an extremal function of the above variational problem, then it is differentiable. This latter led to a major controversy in the 19th century which was settled only by Hilbert.

Let $v=v\left(k, P_{i}\right)$ be the conjugate function of $u$ and set $f=f\left(k, P_{i}\right)=$ $u\left(k, P_{i}\right)+\sqrt{-1} v\left(k, P_{i}\right)$. The conjugate function is locally well defined only up to a constant, so $f$ is multiple-valued in general.

The fundamental group of $C$ has $2 g$ generators $\gamma_{1}, \ldots, \gamma_{2 g}$, and by construction, $f$ satisfies $f\left(\gamma_{j} z\right)=f(z)+p\left(j, k, P_{i}\right)$, where $p\left(j, k, P_{i}\right)$ is independent of $z$.

The functions $f\left(k, P_{i}\right)$ and the constants span a $\left(\sum n_{i}+1\right)$-dimensional vector space $V$ of multiple-valued functions. $g \in V$ is single-valued iff $g\left(\gamma_{j} z\right)$ $=g(z)$ for every $j$. This gives $2 g$ linear conditions, and thus $\Gamma\left(\sum n_{i} P_{i}\right) \geqslant$ $\sum n_{i}+1-2 g$. It turns out that only $g$ of the conditions are independent, giving 3.8.

3.9. CoRollary. Every compact Riemann surface $C$ can be embedded into some $\mathbf{C P}^{n}$, and therefore is algebraic.

Proof. Let $f_{1}, \ldots, f_{n}$ be meromorphic functions on $C$ and consider the map $F: C \rightarrow \mathbf{C P}^{n}, P \rightarrow\left(f_{1}(P): \ldots: f_{n}(P): 1\right) . F$ is defined outside the poles of the $f_{i}$ 's. If $Q \in C$ is a pole of some $f_{i}$, assume that $f_{1}$ has the highest-order pole. Then

$$
F: P \rightarrow\left(f_{1}(P): \ldots: f_{n}(P): 1\right)=\left(1: f_{2}(P) / f_{1}(P): \ldots: 1 / f_{1}(P)\right)
$$

is defined at $Q$; therefore $F$ is defined everywhere.

Assume that with this $F$ we have $F(R)=F(Q)$. Then we pick an $f_{n+1}$ such that it has a pole at $R$ but not at $Q$. Consider

$$
F^{+}: P \rightarrow\left(f_{1}(P): \ldots: f_{n}(P): f_{n+1}(P): 1\right) .
$$

If $F(S) \neq F(T)$ then clearly $F^{+}(S) \neq F^{+}(T)$. Furthermore $F^{+}(R) \neq F^{+}(Q)$. Splitting more and more points apart in this way, it is quite easy to see that eventually we get an injective map. (Here we need that $C$ is compact.)

Conscientious readers might also make the map to be an immersion. A similar technique will work.

3.10. Proof of 3.2. Let $C$ be a smooth curve of genus zero and let $P \in C$. Then $\operatorname{dim} \Gamma(P) \geqslant 2$, so there is a function $f$ on $C$ with one simple pole. This gives a map $f: C \rightarrow \mathbf{C P}^{1}$ which is $1: 1$ near $\infty$, hence everywhere.

3.11. Singularities. Let $f(x, y)=0$ define an algebraic curve in $\mathbf{C}^{2}$. Assume that $f(0,0)=0$. If one of the partials of $f$ is not zero at the origin then by $2.25 f$ defines a manifold near the origin. Otherwise it is singular at the origin. We give some examples:

(i) $x^{2}-y^{2}=0$ : two intersecting lines, called a node.

(ii) $x^{3}-y^{2}=0$, called a cusp.

Note that $p: \mathbf{C} \rightarrow \mathbf{C}^{2}$ given by $t \rightarrow\left(t^{2}, t^{3}\right)$ maps $\mathbf{C}$ onto $\left(x^{3}-y^{2}\right)=0$ in a 1:1 way. The inverse is continuous, but not differentiable at the origin. One can say that the singularity has a parametrization by $\mathbf{C}$. 
(iii) $x^{2 n}-y^{2}=0$. This is the union of $x^{n}-y=0$ and $x^{n}+y=0$, both smooth.

(iv) $x^{3}+x^{6}-y^{4}=0$. This too has a parametrization. Let

$$
\phi(t)=\left(\frac{1}{2}\left(-1+\left(1+4 t^{12}\right)^{1 / 2}\right)\right)^{1 / 3} .
$$

This is a power series convergent for $|t|<2^{-1 / 6} . \Delta \rightarrow \mathbf{C}^{2}, t \rightarrow\left(\phi(t), t^{3}\right)$ parametrizes the singularity, where $\Delta$ is a small disc.

In general every singularity can be parametrized, and we have the following:

3.12. Theorem. Let $C$ be a projective algebraic curve. Then there is a $p$ : $\bar{C} \rightarrow C$ such that $\bar{C}$ is a smooth compact Riemann surface (= projective curve by 3.9) and $p$ is an isomorphism above the smooth points of $C$. Moreover $\bar{C}$ is unique. It will be called the desingularization or normalization of $C$.

3.13. Definition. A possibly singular projective curve will be called rational if its normalization is isomorphic to $\mathbf{C} \mathbf{P}^{1}$. If $f: \mathbf{C P}^{1} \rightarrow D$ is a dominant map and $\bar{D}$ is the normalization of $D$, then one can easily see that $f$ lifts to $\bar{f}: \mathbf{C P}^{1} \rightarrow \bar{D}$. By 3.5(i), $\bar{D} \cong \mathbf{C P}^{1}$ and hence $D$ is rational.

Rational curves will play an important role in the sequel.

\section{Some examples.}

4.1. The simplest algebraic varieties are hypersurfaces. If $f\left(x_{0}, \ldots, x_{n}\right)$ is a homogeneous polynomial of degree $m$, then

$$
F=\left\{(\mathbf{x}) \in \mathbf{C P}^{n} \mid f(\mathbf{x})=0\right\}
$$

is an algebraic variety. As for plane curves, $F$ is irreducible iff $f$ is. By 2.27 it has dimension $n-1$. Essentially by Sard's theorem $F$ is smooth for most choices of $f$. An example that is very easy to work out is $f=x_{0}^{m}+\cdots+x_{n}^{m}$. On the chart $U_{0}$ we have coordinates $z_{i}=x_{i} / x_{0}$ and the equation becomes $1+z_{1}^{m}+\cdots+z_{n}^{m}$. The partial derivatives are $m z_{i}^{m-1}$. All the partials are zero only at the origin, which is not on the hypersurface. Therefore $f$ defines a smooth variety.

4.2. COMPLETE INTERSECTIONS. For homogeneous polynomials $f_{1}, \ldots, f_{k}$, let

$$
V\left(f_{1}, \ldots, f_{k}\right)=\left\{(\mathbf{x}) \in \mathbf{C P}^{n} \mid f_{1}(\mathbf{x})=\cdots=f_{k}(\mathbf{x})=0\right\} .
$$

One can see that for most choices of $f_{1}, \ldots, f_{k}$, the resulting variety is smooth and of dimension $n-k$ (cf. 2.27).

4.3. In $\mathbf{C}^{4}$ with coordinates $x, y, u, v$ let $V$ be the union of the $(x, y)$ and of the $(u, v)$ planes. $V$ is singular at the origin, smooth elsewhere. $V$ can be defined by the equations $x u=x v=y u=y v=0$. It can also be defined by three equations: $x u=y v=x v+y u=0$. It is hard to prove, however, that it cannot be defined by two equations.

By looking at the corresponding $\mathbf{C P}^{3}$, we see that this implies that the union of two skew lines cannot be defined by two equations. It is however still unknown if there exists an irreducible curve $C \subset \mathbf{C P}^{3}$ that cannot be defined by two equations.

4.4. Again in $\mathbf{C}^{4}$ let $V=\{x y-u v=0\}$. This contains the plane $P=\{x=$ $u=0\}$. However one more equation $p$ is not enough to define $P \subset V$. Indeed 
if $P$ is given by $x y-u v=p(x, y, u, v)=0$, then by 2.8

$$
x^{n}=g_{1} \cdot(x y-u v)+f_{1} \cdot p, \quad u^{m}=g_{2} \cdot(x y-u v)+f_{2} \cdot p .
$$

Substituting $y=v=0$, we get

$$
x^{n}=f_{1} \cdot p(x, 0, u, 0), \quad u^{m}=f_{2} \cdot p(x, 0, u, 0) .
$$

This implies that $p(x, 0, u, 0)$ is a constant. On the other hand, $p$ vanishes at the origin; thus $p(x, 0, u, 0) \equiv 0$, a contradiction.

4.5. Products. What is $\mathbf{C P}^{n} \times \mathbf{C P}^{m}$ ? It is clearly not $\mathbf{C P}^{n+m}$. Let $\left(x_{0}: \ldots: x_{n}\right)$ and $\left(y_{0}: \ldots: y_{m}\right)$ be the respective coordinates. On $\mathbf{C P}(n+1)(m+1)-1$ we denote coordinates by $z_{i j}(0 \leqslant i \leqslant n, 0 \leqslant j \leqslant m)$. We define a map from the set $\mathbf{C P}^{n} \times \mathbf{C P}^{m}$ to $\mathbf{C P}^{(n+1)(m+1)-1}$ by

$$
\left(\left(x_{0}: \ldots: x_{n}\right) \times\left(y_{0}: \ldots: y_{m}\right)\right) \rightarrow\left(z_{i j}\right), \text { where } z_{i j}=x_{i} \cdot y_{j} \text {. }
$$

This is clearly an injection. The image can be defined by the obvious equations

$$
z_{s t} z_{p q}=z_{s q} z_{p t}
$$

therefore it is an algebraic variety, called the product of $\mathbf{C P}^{n}$ and $\mathbf{C P}{ }^{m}$. It is exactly what one could expect.

If $\mathbf{C P}^{n}$ is covered by the charts $U_{i} \cong \mathbf{C}^{n}$ and $\mathbf{C P}^{m}$ is covered by the charts $U_{j}^{\prime} \cong \mathbf{C}^{m}$, then $\mathbf{C P}^{n} \times \mathbf{C P}^{m}$ is covered by the charts $U_{i} \times U_{j}^{\prime} \cong \mathbf{C}^{n+m}$. If $U_{i j} \subset \mathbf{C P} \mathbf{P}^{(n+1)(m+1)-1}$ is defined by $z_{i j} \neq 0$, then $U_{i} \times U_{j}^{\prime}=U_{i j} \cap$ $\left(\mathbf{C P}^{n} \times \mathbf{C P}^{m}\right)$.

If $V$ and $W$ are arbitrary algebraic varieties then we define $V \times W$ to be the corresponding subset of $\mathbf{C P}^{n} \times \mathbf{C P}^{m}$. This coincides with any other reasonable definition of the product.

4.6. The product $\mathbf{C P}^{1} \times \mathbf{C P}^{1}$ sits in $\mathbf{C P}^{3}$ defined by one equation $u_{0} u_{1}=$ $u_{2} u_{3}$, which gives a smooth quadric surface. The two families of lines on it are given by $u_{0}=\lambda u_{2}, u_{1}=\lambda^{-1} u_{3}$ and $u_{0}=\mu u_{3}, u_{1}=\mu^{-1} u_{2}$.

4.7. One can easily find the higher-dimensional analogs of elliptic curves. Let $\omega_{1}, \ldots, \omega_{2 n}$ be $\mathbf{R}$-independent vectors in $\mathbf{C}^{n}$. They generate a sublattice $L \subset \mathbf{C}^{n}$ and $\mathbf{C}^{n} / L$ becomes a compact complex manifold, which is homeomorphic to $\left(S^{1}\right)^{2 n}$. As opposed to the case $n=1$, the higher-dimensional ones are not all algebraic. The condition of being algebraic turns out to be very subtle. Let $\Omega=\left(\omega_{1}, \ldots, \omega_{2 n}\right)$ as an $n \times 2 n$ matrix. Then the corresponding $\mathbf{C}^{n} / L$ is algebraic iff there exists a skew symmetric integral $2 n \times 2 n$ matrix $A$ such that

(i) $\Omega A^{t} \Omega=0$ and

(ii) $\sqrt{-1} \Omega A^{t} \bar{\Omega}$ is positive definite.

Now it is difficult to claim that it is natural to single out for study those quotients $\mathbf{C}^{n} / L$ where the above conditions are satisfied. From the point of view of function theory, however, this is naturally forced upon us. A straightforward generalization of 3.9 shows that a compact complex manifold $M$ is algebraic iff for any $p_{1}, \ldots, p_{k} \in M$, there is a meromorphic function $f$ on $M$ such that $f\left(p_{i}\right) \neq f\left(p_{j}\right)$ (and all are finite). Therefore $\mathbf{C}^{n} / L$ is algebraic if and only if there are plenty of $L$-periodic functions on $\mathbf{C}^{n}$.

4.8. HOPF SURFACE. The results mentioned in the previous section are hard to prove. Here we present a simpler example of a nonalgebraic compact complex surface. 
On $\mathbf{C}^{2}-0$ consider the group action $(x, y) \rightarrow(2 x, 2 y)$. The quotient $H=\left(\mathbf{C}^{2}-0\right) / \mathbf{Z}$ is called a Hopf surface. The subset of $\mathbf{C}^{2}-0$ given by $1 \leqslant|x|+|y| \leqslant 2$ is compact and maps onto $H$, so $H$ is compact. Topologically $H$ is $S^{1} \times S^{3}$.

Now consider a meromorphic function on $H$. We can pull it back to $\mathbf{C}^{2}-0$ to get a meromorphic function $f(x, y)$ such that $f(x, y)=f(2 x, 2 y)$. By the Levi extension theorem $f$ is also meromorphic at $(0,0)$. Therefore we can write $f$ as the quotient of two power series, $f=g / h$.

Restricting $f$ to the line $y=\lambda x$ we get

$$
p(x)=f(x, \lambda x)=g(x, \lambda x) / h(x, \lambda x),
$$

which is meromorphic in $x$ provided $h(x, \lambda x) \not \equiv 0$. This is satisfied for all but finitely many values of $\lambda$. We also have $p(2 x)=p(x)$. If we look at the Laurent expansion of $p(x)=\sum a_{i} x^{i}$ this implies $a_{i}=2^{i} a_{i}$, hence $p(x)$ is constant. Therefore $f$ is constant along all the lines $y=\lambda x$. One can easily conclude that $f$ is a rational function of $x / y$.

Anyhow we can see that $H$ is covered by the images of the lines $y=\lambda x$, and every meromorphic function on $H$ is constant along these curves. Therefore $H$ cannot be algebraic.

It is worthwhile to note that for $n=1$ the analogous construction yields the elliptic curve $E_{\tau}$ with $\tau=(-1 / 2 \pi i) \log 2$.

5. Maps between algebraic varieties. This chapter deals with various ways of describing maps between algebraic varieties. To avoid confusion it is important to note that we will consider functions and maps that are not everywhere defined. This is in accordance with tradition; no one had any qualms about claiming that $1 / z$ was a function on $\mathbf{C}$. The term morphism or regular map will always refer to a map that is everywhere defined. It will be symbolized by a solid arrow $\rightarrow$. A dotted arrow $\rightarrow$ will indicate a map that may not be defined everywhere.

5.1. RegulaR FUnCTIONS. Let $V \subset \mathbf{C}^{n}$ be a closed algebraic subvariety. Which should be the basic functions on $V$ ? Since we are doing algebraic geometry, we should consider the polynomials on $\mathbf{C}^{n}$. There are two ways to go from $\mathbf{C}^{n}$ to $V$. One can consider the restrictions of polynomials, or one can consider those functions that are locally restrictions of polynomials. Fortunately these two notions agree. Such a function is called regular on $V$.

5.2. RATIONAL FUNCTIONS. Frequently it is necessary to work with quotients of polynomials. These are called rational functions on $\mathbf{C}^{n}$. For $V \subset \mathbf{C}^{n}$ there are again two a priori different notions, but again they agree. The restriction of a rational function $f$ from $\mathbf{C}^{n}$ to $V$ is called a rational function on $V$. For this to make sense, we must require that none of the irreducible components of $V$ is contained in the polar set of $f$. We want our functions to be defined most of the time.

A rational function $f$ is called regular at $v \in V$ if there are polynomials $g$ and $h$ such that $f=g / h$ and $h(v) \neq 0$. A rational function is called regular on $V$ if it is regular at each point. One can see that a regular rational function is a regular function. 
5.3. EXAMPLES. (i) $x / y$ is a rational function on $\mathbf{C}^{2}$; it is regular at $(x, y)$ iff $y \neq 0$.

(ii) Let $L \subset \mathbf{C}^{2}$ be the line $x=y$, and let $f=(x / y) \mid L$. Then $f$ is rational on $L$. Moreover since $f \equiv 1$, it is even regular.

(iii) Let $V=\left(x^{2}-y^{3}=0\right) \subset \mathbf{C}^{2}$. Let $f=(x / y) \mid V . f$ is a rational function, regular outside $(0,0)$. One can easily see that if we declare $f(0,0)=0$, then $f$ is continuous on $V$. Despite this, $x / y$ is not regular at $(0,0)$. Indeed, assume that

$$
x / y=a(x, y) / b(x, y) \text { and } b(0,0) \neq 0 \text {. }
$$

Then $x b(x, y)-y a(x, y)$ is zero on $V$, hence divisible by $x^{2}-y^{3} . b(x, y)$ has a nonzero constant term, hence the coefficient of $x$ in $x b(x, y)-y a(x, y)$ is not zero. Therefore it cannot be divisible by $x^{2}-y^{3}$.

It is also worthwile to note that $f^{2}=x^{2} / y^{2}=y \mid V$, and therefore it is regular. This peculiar behavior comes from the singularity of $V$ at the origin, and is the source of many inconveniences. Varieties for which this does not occur deserve a name.

5.4. Definition. Let $V \subset \mathbf{C}^{n}$ be an algebraic variety and $v \in V$. $V$ is said to be normal at $v \in V$ if every rational function bounded in some neighborhood of $v$ is regular at $v . V$ is called normal if it is normal at every point. In particular if $V$ is normal at $v$, then a rational function is regular at $v$ iff it is continuous at $v$.

Riemann's extension theorem says that $\mathbf{C}$ is normal. From this it easily follows that smooth points are normal in all dimensions.

5.5. Proposition. Let $C$ be an algebraic curve. Then $C$ is normal iff $C$ is smooth.

Proof. Assume that $C$ is normal. Let $p: \bar{C} \rightarrow C$ be the desingularization (3.12). Given $c \in C$, let $p^{-1}(c)=\left\{c_{1}, \ldots, c_{k}\right\}$. Let $f$ be a rational function on $\bar{C}$ which has a simple zero at $c_{1}$ and takes nonzero finite values at $c_{2}, \ldots, c_{k}$. One can easily see that $f \circ p^{-1}$ is a rational function on $C$. It is bounded near $c$, and hence regular at $c$. Thus $p^{-1}(c)=\left\{c_{1}\right\}$ and $f \circ p^{-1}$ maps a neighborhood of $c \in C$ into a neighborhood of the origin in $\mathbf{C}$. Therefore $c \in C$ is a smooth point.

5.6. Corollary. Let $V$ be a normal variety. Then $\operatorname{dim} \operatorname{Sing} V \leqslant \operatorname{dim} V-2$.

IDEA OF PROOF. We can view an $n$-dimensional variety $V$ as an $(n-1)$ dimensional family of curves. If $\operatorname{dim} \operatorname{Sing} V=n-1$, then each of these curves is singular. In the proof of 5.5 we could make everything depend on $n-1$ parameters and conclude as above.

5.7. ExAmple. Let $f: \mathbf{C}^{2} \rightarrow \mathbf{C}^{7}$ be given by $(x, y) \mapsto\left(x^{2}, x y, y^{2}, x^{3}\right.$, $\left.x^{2} y, x y^{2}, y^{3}\right)$. Let $V=f\left(\mathbf{C}^{2}\right)$. Then $V$ is smooth outside the origin, but not normal, since $x \circ f^{-1}$ is not regular.

There is one important case, however, when the converse of 5.6 is true. Unfortunately, I don't know any simple proof.

5.8. THEOREM. Let $F=(f=0) \subset \mathbf{C}^{n}$ be a hypersurface. Then $F$ is normal iff $\operatorname{dim} \operatorname{Sing} F \leqslant \operatorname{dim} F-2$.

There is a very useful extension theorem that holds for normal varieties. 
5.9. Hartogs' Theorem. Let $V$ be a normal variety and let $W \subset V$ be a subvariety such that $\operatorname{dim} W \leqslant \operatorname{dim} V-2$. Let $f$ be a regular function on $V-W$. Then $f$ extends to a regular function on $V$.

Proof. Assume for simplicity that $W$ is a single point $w$. Let $V \subset \mathbf{C}^{n}$ and let $B \subset \mathbf{C}^{n}$ be a small ball around $w$. If $v \in V \cap B$ then by repeated hyperplane cuts we get an algebraic curve $C \subset V$ through $v$ that avoids $w$. Let $D=B \cap C$.

$|f|$ is bounded on the compact set $\partial B \cap V$ by some constant $M . f \mid D$ is holomorphic; thus by the maximum principle

$$
|f(v)| \leqslant \max \{|f(z)|: z \in \partial D\} \leqslant M .
$$

So $|f|$ is bounded near $w$, and therefore $f$ is regular at $w$.

5.10. Definition. If $W \subset \mathbf{C P}^{n}$ is an arbitrary algebraic variety, then it is covered by charts $V_{i}=W \cap U_{i}$. The notions of rational and regular functions and of normality can then be defined using this covering.

5.11. Proposition. Let $V$ be an irreducible projective variety and $f$ a regular function on $V$. Then $f$ is constant.

Proof. Only for $V$ smooth. Then $V$ is a compact complex manifold and $f$ is holomorphic on $V .|f|$ achieves its maximum since $V$ is compact, hence by the maximum principle $f$ is constant.

5.12. First DEFinition of MAPS. A map from $V$ to some $\mathbf{C P}^{n}$ should be given by coordinate functions. Pick rational functions $f_{1}, \ldots, f_{n}$ on $V$ and let

$$
F: V \rightarrow \mathbf{C P}^{n}, \quad v \rightarrow\left(f_{1}(v): \ldots: f_{n}(v): 1\right)
$$

be the map. This is the approach used in 3.9. $F$ is certainly defined whenever each of the $f_{i}$ 's is defined. But $F$ is defined some other places too; to wit, $F$ is defined at $v$ iff there is a $g$, regular at $v$ such that all the $f_{i} g$ are regular at $v$ and $\left(f_{1} g: \ldots: f_{n} g: g\right)$ is not identically zero at $v$. Instead of saying that $f$ is defined at $v$ we shall say that it is regular at $v$.

If $h$ is a rational function on $\mathbf{C P}^{n}$ and $F(V)$ is not contained in its polar locus, then $F^{*} h$ is a rational function on $V$. If $F$ is regular at $v$ and $h$ is regular at $F(v)$, then $F^{*}(h)$ is regular at $v$.

The following results show some nice topological properties of regular maps.

5.13. TheOREM (Dimension FORMUla). Let $f: V \rightarrow W$ be a regular map. Then for any $w \in W, f^{-1}(w)$ is either empty or

$$
\operatorname{dim} f^{-1}(w) \geqslant \operatorname{dim} V-\operatorname{dim} W .
$$

If $w \in W$ is sufficiently general, then equality holds.

Proof. Assume for simplicity that $w \in W$ is smooth. If $z_{1}, \ldots, z_{k}$ are local coordinates at $w$, then $f^{-1}(w)$ is defined by $f^{*} z_{1}=\cdots=f^{*} z_{k}=0$. Thus 2.27 yields the required inequality.

If $v \in V$ is sufficiently general, then a small neighborhood of $v$ is diffeomorphic to the product of a neighborhood of $f(v) \in W$ and a neighborhood of $v$ in $f^{-1}(f(v))$. This proves the last claim. 
With a little bit of work, this gives the following:

5.14. Corollary. Let $f: V \rightarrow W$ be a regular map. Then $w \rightarrow \operatorname{dim} f^{-1}(w)$ is upper semicontinuous on $W$.

Although theoretically 5.12 is the easiest way to define maps, it is the least convenient to work with.

5.15. SECOND DEFINITION OF MAPS. This is based on a better understanding of $\mathbf{C P}^{n}$. A point in $\mathbf{C P}^{n}$ can be given by homogeneous coordinates, but these are not unique. To make it unique, a point in $\mathbf{C P}^{n}$ is given by a line in $\mathbf{C}^{n+1}$; if $f: V \rightarrow \mathbf{C P}^{n}$ is a map, then this associates to each $v \in V$ a line in $\mathbf{C}^{n+1}$. So the map can be most conveniently described by a subset

$$
L \subset V \times \mathbf{C}^{n+1}
$$

such that for each $v \in V, L \cap\left(\{v\} \times \mathbf{C}^{n+1}\right)$ is a line $L_{v}$, and this line "varies algebraically" with $v$. Conversely, any such subset $L$ defines a map into $\mathbf{C P}^{n}$.

It is more convenient to consider the dual set-up: instead of $L_{v} \subset \mathbf{C}^{n+1}$ we look at $\left(\mathbf{C}^{n+1}\right)^{*} \rightarrow L_{v}^{*}$. In this case $L^{*}$ is an algebraically varying family of quotient lines on $V$, and identifying $\left(\mathbf{C}^{n+1}\right)^{*} \cong \mathbf{C}^{n+1}$ gives a map

which is linear on each $\{v\} \times \mathbf{C}^{n+1}$.

$$
q: V \times \mathbf{C}^{n+1} \rightarrow L^{*},
$$

If $e \in \mathbf{C}^{n+1}$ then $v \rightarrow(v, e) \rightarrow q(v, e)$ gives a map from $V$ to $L^{*}$, denoted by $q_{e}$. It is clearly regular and $q_{e}(v) \in L_{v}^{*}$. Such a map is called a section of $L^{*}$. If $e_{0}, \ldots, e_{n}$ is a basis of $\mathbf{C}^{n+1}$, let $q_{0}, \ldots, q_{n}$ be the corresponding sections. Since $q:\{v\} \times \mathbf{C}^{n+1} \rightarrow L_{v}^{*}$ is onto for each $v$, at least one of the $q_{i}$ 's is not zero at any $v \in V$.

Conversely if we pick $n+1$ sections $s_{0}, \ldots, s_{n}$ of $L^{*}$ such that at any point of $V$ at least one of them is not zero, then we can define $s: V \times \mathbf{C}^{n+1} \rightarrow L^{*}$ by $s\left(v, \sum a_{i} e_{i}\right)=\sum a_{i} s_{i}(v)$. Thus these sections define a map $V \rightarrow \mathbf{C P}$.

Now we can define in general: let $L$ be an "algebraically varying" family of lines over $V$ and let $s_{0}, \ldots, s_{n}$ be sections of $L$. Then these define a map $V \rightarrow \mathbf{C P} \mathbf{P}^{n}$. This map is certainly defined at $v$ if one of the $s_{i}(v)$ 's is not zero.

An alternate way to look at this is as follows. The $s_{i}(v)$ 's are elements of $L_{v}$, which is a one-dimensional $\mathbf{C}$-vector space. Therefore the sequence $s_{0}(v), \ldots, s_{n}(v) \in \mathbf{C}^{n+1}$ is defined only up to a constant factor, hence it defines a point in $\mathbf{C P}^{n}$.

5.16. THIRD DEFINITION OF MAPS. This is again a standard way of looking at maps: studying their graphs. Let $F: V \rightarrow W$ be a map that is defined at every point of $V$. Then its graph $\Gamma(F) \subset V \times W$ is closed and is easily seen to be an algebraic subvariety of $V \times W$. In general, however, the graph is not closed, and it is very useful to study its closure. By 2.22 the closure is an algebraic subvariety of $V \times W$.

Now let $\Gamma \subset V \times W$ be a closed subvariety. How can one recognize that $\Gamma$ is the graph of a map? Let $p$ resp. $q$ be the two projections of $V \times W$ onto $V$ resp. $W$. If $\Gamma$ is the graph of a map $F$, then $F(v)=q\left(p^{-1}(v)\right)$ whenever $F$ is defined. This means that $p^{-1}(v)$ is only one point for most $v \in V$. Conversely, assume that $\Gamma$ is such that $p: \Gamma \rightarrow V$ is onto and $1: 1$ at most points. Then it is not difficult to see that $\Gamma$ is the graph of an algebraic map. 
5.17. ZARISKI CONNECTEDNESS THEOREM. Let $p: \Gamma \rightarrow V$ be a proper regular map between irreducible algebraic varieties. Assume that $p^{-1}: V \rightarrow \Gamma$ is a rational map (i.e., $\Gamma$ is the graph of some map $V \rightarrow W$ ) and that $V$ is normal. Then $p^{-1}(v)$ is connected for every $v \in V$.

Proof. For $V$ smooth only. If $p^{-1}$ is regular at $v$, then $p^{-1}(v)$ is a single point; thus we have to look at the set $Z \subset V$ where $p^{-1}$ is not regular. 2.21 implies that $p^{-1}(V-Z)$ is dense in $\Gamma$. Let $z \in Z$ and let $S_{\varepsilon} \subset V$ be a small $2 n-1$ sphere around $z(n=\operatorname{dim} V)$. Real $\operatorname{dim} S_{\varepsilon} \cap Z=$ real $\operatorname{dim} Z-1 \leqslant$ $2 n-3$ and therefore $S_{\varepsilon} \cap(V-Z)$ is connected. Since $p^{-1}(v)$ is the limit of $p^{-1}\left(S_{\varepsilon} \cap(V-Z)\right)$ as $\varepsilon \rightarrow 0, p^{-1}(v)$ must be connected.

If $V$ is not smooth then the topology of $V$ is less understood and the hard part is to prove that $S_{\varepsilon} \cap(V-Z)$ is connected.

5.18. Corollary. With the above notation $p^{-1}$ is regular at $v$ iff $p^{-1}(v)$ is a single point.

Proof. The necessity is clear. Conversely assume that $p^{-1}(v)$ is a single point. Then by $5.14, \operatorname{dim} p^{-1}(\cdot)=0$ in a neighborhood of $v$. Thus by $5.17 p^{-1}$ is single-valued and continuous in a neighborhood of $v$, hence regular at $v$.

5.19. Corollary. Let $V, W$ be projective varieties and assume that $V$ is normal. Let $f: V \rightarrow W$ be a map. Then there is a subset $Z \subset V$ such that $\operatorname{dim} Z \leqslant \operatorname{dim} V-2$ and $f$ is regular on $V-Z$.

Proof. Let $\Gamma \subset V \times W$ be the closure of the graph. Then $f=q \circ p^{-1}$ and we need to find a $Z$ such that $p^{-1}$ is regular on $V-Z$. Let $Z=\{v \in V$ : $p^{-1}(v)$ is not a point $\}$. This is clearly a closed subset; one can even see that it is algebraic. $E=p^{-1}(Z)$ is a subvariety of $\Gamma$.

Since $p^{-1}(v)$ is connected, it is either a point or has dimension at least one. Therefore $\operatorname{dim} E \geqslant 1+\operatorname{dim} Z . E$ is a proper subvariety of $\Gamma$, hence $\operatorname{dim} E \leqslant$ $\operatorname{dim} \Gamma-1$. This gives the required inequality.

5.20. Remark. Let $V, W$ be complex manifolds. The previous three definitions of maps make sense in this case too. Instead of polynomials one has to consider power series, and instead of rational functions, meromorphic functions. For algebraic varieties we get two different notions of maps this way, one algebraic and one analytic. For projective varieties, however, the two notions agree:

5.21. Theorem. Let $V$ and $W$ be projective algebraic varieties. Then any meromorphic map from $V$ to $W$ is algebraic. In particular, any meromorphic function on $V$ is rational.

Proof. Let $\Gamma \subset V \times W$ be the closure of the graph of a meromorphic map. One can see that it is a closed analytic subvariety of $V \times W \subset \mathbf{C P}(n+1)(m+1)-1$. By Chow's theorem (2.11) it is therefore an algebraic subvariety; thus, as we remarked in 5.16, the map is algebraic.

Meromorphic functions are maps from $V$ to $\mathbf{C P}^{1}$, hence the last claim.

5.22. REMARK. A fourth, very unusual approach to maps will be given in the next chapter.

The following result shows an unusual and useful feature of algebraic maps. 
5.23. RIGIDITY THEOREM. Let $U, V, W$ be algebraic varieties (or complex manifolds). Assume that $V$ is projective (compact), and that $U$ is connected. Let $f: U \times V \rightarrow W$ be a regular (holomorphic) map. Assume that $f\left(\left\{u_{0}\right\} \times V\right)=$ point for some $u_{0} \in U$. Then $f(\{u\} \times V)=$ point for every $u \in U$.

Proof. Let $Z \subset U$ be the set of those points $u \in U$ such that $f(\{u\} \times V)$ = point. $Z$ is clearly closed, thus $Z=U$ follows once we establish that it is also open. Let $u \in Z$ and let $u^{\prime}$ be near $u$. Since $V$ is compact, $f\left(\left\{u^{\prime}\right\} \times V\right)$ is near $f(\{u\} \times V)=$ point, and therefore it is contained in a small neighborhood of that point. Therefore local coordinates on this neighborhood give global regular functions on $\left\{u^{\prime}\right\} \times V$. By 5.11 these are constants, hence $f\left(\left\{u^{\prime}\right\} \times V\right)$ is a point.

One surprising consequence will be given after a definition:

5.24. Definition. A complex Lie group is a complex manifold with a group structure such that the group operations are holomorphic.

5.25. Proposition. A connected, compact complex Lie group is commutative.

Proof. Let $G$ be the group, and let $f: G \times G \rightarrow G$ be given by $f(a, b)=$ $b^{-1} a b$. We have $f(\{e\} \times G)=\{e\}$, where $e$ is the identity. Hence by 5.23, $f(\{a\} \times G)=$ point. Therefore $b^{-1} a b=e^{-1} a e=a$, and $a b=b a$.

6. Topology of algebraic varieties. In this chapter we discuss some simple but powerful topological properties of algebraic varieties. This will provide a natural introduction to Mori's program.

6.1. BASIC TECHNICAL FACT. The underlying topological space of an algebraic variety can be triangulated. If $X \subset Y$ is a closed subvariety, then there is a triangulation of $Y$ such that $X$ is the union of simplices. Therefore $X^{k} \subset Y$ has a homology class $[X] \in H_{2 k}(Y, \mathbf{Z})$.

6.2. EXAMPLE. Let $f$ be a meromorphic function on $Y$ with zeros $Z_{0}$ and poles $Z_{\infty} \subset Y$. Pick a path between 0 and $\infty$ in $\mathbf{C P}^{1}$; its preimage has boundary $Z_{0}-Z_{\infty}$; therefore $\left[Z_{0}\right]=\left[Z_{\infty}\right]$.

For instance, if $Y=\mathbf{C P}^{n}$ and $g$ is a degree $k$ homogeneous polynomial defining a hypersurface $G$, then take $f=g / x_{0}^{k}$ and obtain that $[G]=k[H]$, where $H$ is a hyperplane.

6.3. FUndAMENTAL FACT. A complex manifold has a natural orientation.

Proof. A complex manifold is locally like $\mathbf{C}^{n}$, and so we have to show that $\mathbf{C}^{n}$, viewed as a $2 n$-dimensional real vector space, carries a natural orientation.

Pick a basis $e_{1}, \ldots, e_{n}$ in $\mathbf{C}^{n}$. Then $e_{1}, \ldots, e_{n}, i e_{1}, \ldots, i e_{n}$ is a real basis of $\mathbf{C}^{n}$ and hence determines an orientation. What if we start with a different basis? Let $A+i B \in \operatorname{GL}(n, \mathrm{C})(A, B$ real matrices) be the matrix of the change of basis. Then the real basis is changed by the matrix $\left(\begin{array}{cc}A & B \\ -B & A\end{array}\right)$ and we need to show that its determinant is positive.

1st Proof.

$$
\left(\begin{array}{cc}
1 & i \\
i & 1
\end{array}\right)\left(\begin{array}{cc}
A & B \\
-B & A
\end{array}\right)\left(\begin{array}{cc}
1 & i \\
i & 1
\end{array}\right)^{-1}=\left(\begin{array}{cc}
A-i B & 0 \\
0 & A+i B
\end{array}\right)
$$


hence

$$
\operatorname{det}\left(\begin{array}{cc}
A & B \\
-B & A
\end{array}\right)=|\operatorname{det}(A+i B)|^{2}>0
$$

2ND PROOF.

$$
\operatorname{det}\left(\begin{array}{cc}
A & B \\
-B & A
\end{array}\right)
$$

is a continuous nowhere zero function on $\operatorname{GL}(n, \mathbf{C})$, equal to 1 at the identity. Since $\mathrm{GL}(n, \mathbf{C})$ is connected, it is everywhere positive.

6.4. Corollary. Positivity of INTERSECTION. Let $Y$ be a complex manifold, $U, V \subset Y$ be subvarieties intersecting transversally. Let $A_{i}$ be the components of $U \cap V$. Then it is known from topology that $[U] \cap[V]=\sum \varepsilon_{i}\left[A_{i}\right]$, where $\varepsilon_{i}= \pm 1$ depending on the orientations of $U, V$ and $Y$ along $A_{i}$. Since we have everything canonically oriented we have: $[U] \cap[V]=\Sigma\left[A_{i}\right]$.

6.5. Corollary. Let $Y$ be a projective variety and let $X^{k} \subset Y$ be a closed subvariety. Then $[X] \in H_{2 k}(X, \mathbf{Q})$ is never zero.

Proof. We embed $Y \subset \mathbf{C P}^{n}$ and then it suffices to see that $[X] \in$ $H_{2 k}\left(\mathbf{C P}^{n}, \mathbf{Q}\right)$ is not zero. Let $x \in X$ be a general point and let $L^{n-k}$ be a general $(n-k)$-plane through $x$. Then $X \cap L$ is a discrete set of points $x=x_{1}, x_{2}, \ldots, x_{m}$ and so

$$
[X] \cap[L]=[x]+\cdots+\left[x_{m}\right]=m[\mathrm{pt}] \in H_{0}\left(\mathbf{C P}^{n}, \mathbf{Q}\right) \cong \mathbf{Q} .
$$

Thus $[X] \cap[L] \neq 0$ and this implies that $[X] \neq 0$.

6.6. REMARKS. (i) The same argument shows that if $X_{i}^{k} \subset Y$ are subvarieties, then $\sum a_{i}\left[X_{i}\right] \neq 0$ for $a_{i}>0$.

(ii) For nonprojective complex manifolds the corollary can fail (see 12.11).

6.7. Definition. For a smooth projective variety $X$ let $N E(X) \subset H_{2}(X, \mathbf{R})$ be the set of positive linear combinations of homology classes of curves on $X$. This is obviously a subcone of the vector space $H_{2}(X, \mathbf{R})$. By 6.6(i) $0 \notin N E(X)$, hence it contains no lines. This is called the cone of curves of $X$. It is usually easier to work with its closure $\overline{N E}(X)$ which is called the closed cone of curves. $(N E(X)$ is a quite unfortunate but standard notation.)

It will follow from 7.15 that $\overline{N E}(X)$ contains no line either.

6.8. Definition. (i) Let $V \subset \mathbf{R}^{n}$ be a convex cone and let $W \subset V$ be a subcone. $W$ is said to be extremal if $u, v \in V, u+v \in W \Rightarrow u, v \in W$. Geometrically: $V$ lies on one side of $W$.

(ii) A one-dimensional subcone will be called a ray.

(iii) It is easy to see that if a closed convex cone $V$ contains no lines, then it is the convex hull of its extremal rays. $V$ is said to be locally finitely generated at $v \in V$ if only finitely many extremal rays intersect a small enough neighborhood of $v$. This notion is interesting only for boundary points.

Now we are ready to outline a fourth approach to maps between projective varieties. Although it is a rather straightforward idea, it appeared first only in Hironaka's thesis, and was used successfully first by Mori. 
6.9. Definition. Let $X, Y$ be projective varieties and $f: X \rightarrow Y$ a map. Let $N E(f)$ or $N E(X / Y)$ be the subcone of $N E(X)$ generated by those curves $C \subset X$ such that $f(C)=$ point. I will call this the kernel cone of $f$. Its closure is denoted by $\overline{N E}(f)$.

6.10. Proposition. (Notation as in 6.9.) (i) For a curve $C, f(C)=$ point $\Leftrightarrow$ $[C] \in N E(f)$.

(ii) $N E(f)$ is extremal.

Proof. (i) $(\Rightarrow)$ holds by definition. If $[C] \in N E(f)$, then $[C]=\sum a_{i}\left[C_{i}\right]$ such that $f\left(C_{i}\right)=$ point. Therefore $[f(C)]=\sum a_{i}\left[f\left(C_{i}\right)\right]=0 \in H_{2}(Y, \mathbf{R})$. Then since by $6.5, f(C)$ cannot be a curve, it must be a point.

(ii) If $u=\sum a_{i}\left[C_{i}\right], v=\sum b_{j}\left[D_{j}\right]$, and $u+v \in N E(f)$, then as above we obtain that $\sum a_{i}\left[f\left(C_{i}\right)\right]+\sum b_{j}\left[f\left(D_{j}\right)\right]=0$. Hence $f\left(C_{i}\right)$ and $f\left(D_{j}\right)$ are points; thus $u, v \in N E(f)$.

Now we come to the starting point of Mori's program.

6.11. FUNDAMENTAL TRIVIALITY OF MORI'S PROGRAM. Let $X$ be a projective variety and $f: X \rightarrow Y$ a map onto some normal projective variety. Assume that $f$ has only connected fibers. Then $f$ is uniquely determined by its kernel cone $N E(f)$.

Proof. The recipe to get $f$ is the following: if $x, y \in X$, then $f(x)=f(y)$ iff there is a chain of curves $\left\{C_{i}\right\}$ connecting $x$ and $y$ such that $\left[C_{i}\right] \in N E(f)$.

If $f(x)=f(y)$, then such a chain can be found since $f^{-1}(f(x))$ is connected. If such a chain can be found, then $f\left(\cup C_{i}\right)=\bigcup f\left(C_{i}\right)$ is a finite set of points by 6.10(i). Since $\cup C_{i}$ is connected it must be one point; thus $f(x)=f(y)$.

I want to emphasize the necessity of the projectivity condition on the image of $f .6 .10(\mathrm{i})$ and 6.11 are false without this assumption.

6.12. Definition. Let $V \subset N E(X)$ be a closed subcone. We say that $V$ can be contracted if there is a normal variety $Y$ and a surjective map $f: X \rightarrow Y$ such that $f$ has connected fibers and $V=N E(f)$. The map $f$ (unique by 6.11) will be called the contraction map of $V$.

6.13. REMARK. If $g: X \rightarrow Z$ is an arbitrary map, then it is intuitively quite clear that it can be factored into $f: X \rightarrow Y, h: Y \rightarrow Z$ where $f$ has connected fibers, $Y$ is normal, and $h$ has finite fibers. $Y$ is projective if $Z$ is. This is the so-called Stein factorization.

6.14. QUESTIONS. These are of course innumerable. How can one describe $N E(X)$ ? Which subcones correspond to maps? How can one read off properties of $f$ from $N E(f)$ ?

Very little is known in general. In some cases, however, a beautiful answer can be given. This will be the heart of Mori's program.

7. Vector bundles and the canonical bundle. We already considered line bundles passingly in 5.15. Because of their importance in describing algebraic maps, we shall investigate them in more detail.

7.1. Definition. The idea of vector bundles is that we have an algebraically varying family of vector spaces. Technically the following definition seems better:

Let $X$ be an algebraic variety. A vector bundle over $X$ is an algebraic variety $V$ and a regular map $p: V \rightarrow X$ with the following property. 
For any $x \in X$ there is an open algebraic subset $U$ containing $x$ and an algebraic isomorphism $g: U \times \mathbf{C}^{n} \rightarrow p^{-1}(U)$ such that $p \circ g(u, e)=u$ for any $u \in U, e \in \mathbf{C}^{n}$. Furthermore if $g_{i}: U_{i} \times \mathbf{C}^{n} \rightarrow p^{-1}\left(U_{i}\right)$, for $i=1$ and 2, are any two maps and $x \in U_{1} \cap U_{2}$, then the two vector space structures $g_{i}:\{x\} \times \mathbf{C}^{n}$ $\rightarrow p^{-1}(x)$ induced on $p^{-1}(x)$ are the same.

A slightly different way of giving this definition is to consider $V$ to be patched together from the pieces $U_{i} \times \mathbf{C}^{n}$ with the help of transition functions:

$$
g_{i j}=g_{i} \circ g_{j}^{-1}:\left(U_{i} \cap U_{j}\right) \times \mathbf{C}^{n} \rightarrow\left(U_{i} \cap U_{j}\right) \times \mathbf{C}^{n} .
$$

The second condition above is equivalent to the requirement that $g_{i} \circ g_{j}^{-1}$ is a matrix-valued invertible regular function. $n$ is called the rank of the vector bundle.

In an analogous way one can define analytic vector bundles. A variant of Chow's theorem yields that any analytic vector bundle on a projective algebraic variety is algebraic.

7.2. Definition. The usual vector space operations can be applied fiberwise to vector bundles. Therefore one can define direct sums, tensor products and determinant bundles of algebraic vector bundles.

If $p_{i}: V_{i} \rightarrow X$ are vector bundles on $X$, then a vector bundle homomorphism between them is a regular map $f: V_{1} \rightarrow V_{2}$ such that $p_{1}\left(v_{1}\right)=p_{2}\left(f\left(v_{1}\right)\right)$ for $v_{1} \in V_{1}$ and each $f: p_{1}^{-1}(x) \rightarrow p_{2}^{-1}(x)$ is linear.

A sequence of vector bundle homomorphisms is called exact if it is exact above each $x \in X$ as maps of vector spaces. If

$$
0 \rightarrow V_{1} \rightarrow V_{2} \rightarrow V_{3} \rightarrow 0
$$

is an exact sequence, then we can take determinants to get

$$
\operatorname{det} V_{2} \cong \operatorname{det} V_{1} \otimes \operatorname{det} V_{3} \text {. }
$$

7.3. Definition. If $p: V \rightarrow X$ is an algebraic vector bundle and $f: Y \rightarrow X$ is a regular map, then we can define a vector bundle $f^{*} p: f^{*} V \rightarrow Y$ as follows. If $V$ is given by patches $U_{i} \times \mathbf{C}^{n}$ and transition functions $g_{i j}$, then $f^{*} V$ is given by patches $f^{-1}\left(U_{i}\right) \times \mathbf{C}^{n}$ and transition functions $g_{i j} \circ f$. If $f$ is an inclusion, then $f^{*} V$ is called the restriction of $V$ to $Y$, and is denoted by $V \mid Y$.

7.4. Definition. Let $p: V \rightarrow X$ be an algebraic vector bundle. A section of $V$ is a regular map $s: X \rightarrow V$ such that $p \circ s=$ id. These are called global sections of $V$. They form a vector space under pointwise addition, denoted by $\Gamma(X, V)$. A section defined only on an open subset $U \subset X$ is called a local section.

A rational section of $V$ is a rational map $t: X \rightarrow V$ such that $p \circ t=$ id. If $V$ is an algebraic vector bundle, then it always has rational sections. To see this choose a $U_{i}$ and an $e \in \mathbf{C}^{n}$ and let $t(u)=g_{i}(u, e) \in V$. This $t$ is regular on $U_{i}$, and on $U_{j}$ it is given by $u \mapsto g_{j i} \circ g_{i}$, which is a rational function. By a slight abuse of terminology I will frequently refer to a rational section as meromorphic.

The following easy consequence of 5.9 will be very useful.

7.5. Proposition. Let $X$ be a normal variety and $Y \subset X$ a subvariety such that $\operatorname{dim} Y \leqslant \operatorname{dim} X-2$. Let $V$ be a vector bundle over $X$. Then any section $s$ of $V \mid X-Y$ extends to a section $\bar{s}$ of $V$. 
Proof. If $\bar{s}$ exists then it is clearly unique. Therefore the extension problem is local on $X$. Let $x \in U \subset X$ be a small neighborhood such that $V \mid U$ is of the form $U \times \mathbf{C}^{n}$. The section $s \mid U-Y$ now corresponds to $n$ regular functions on $U-Y$. By 5.9 they all extend to regular functions on $U$, hence give an extension $\bar{s}\left|U: U \rightarrow U \times \mathbf{C}^{n} \cong V\right| U$. This proves the proposition.

7.6. EXAMPLES. (i) For every $n$ there is the trivial bundle $X \times \mathbf{C}^{n}$.

(ii) We already considered the example of $\mathbf{C P}^{n}$ in 5.9. Each point corresponds to a line and this gives a line subbundle $L \subset \mathbf{C} \mathbf{P}^{n} \times \mathbf{C}^{n+1}$. This is called $\mathcal{O}(-1)$. Its dual, a quotient of $\mathbf{C} \mathbf{P}^{n} \times \mathbf{C}^{n+1}$, is called $\mathcal{O}(1)$. Its $k$ th tensor power is denoted by $\mathcal{O}(k)$. We shall see in 7.17 that $\mathcal{O}(k): k \in \mathbf{Z}$ are all the line bundles on $\mathbf{C P}^{n}$.

(iii) If $f: X \rightarrow \mathbf{C P}^{n}$ is a regular map, then $f^{*} \mathcal{O}(1)$ is a line bundle on $X$. As we saw in 5.15, this line bundle along with $n+1$ sections determines the map $f$. Due to the importance of maps into projective spaces, it will be important to find ways of deciding whether a line bundle on $X$ is of the form $f^{*} \mathcal{O}(1)$ for some $f$.

(iv) Let $X$ be an algebraic variety covered by charts $X=U_{1} \cup \cdots \cup U_{n}$ and let $H \subset X$ be a closed subvariety such that on each chart $U_{i}$ there is a regular function $f_{i}$ such that $H \cap U_{i}=\left(f_{i}=0\right)$. Assume furthermore that $f_{i}$ has a simple zero along each component of $H \cap U_{i}$. If $X$ is smooth and $\operatorname{dim} H=$ $\operatorname{dim} X-1$, then these conditions are automatically satisfied (2.28(i)). We will construct a line bundle $\mathcal{O}(H)$ on $X$ and a section $s: X \rightarrow \mathcal{O}(H)$ such that $H=(s=0)$. It will be convenient to introduce an extra chart $U_{0}=X-H$, and pick $f_{0} \equiv 1 . \mathcal{O}(H)$ is given by transition functions

$$
g_{i j}: U_{i} \times \mathbf{C} \rightarrow U_{j} \times \mathbf{C} ; \quad g_{i j}(x, z)=\left(x, \frac{f_{j}(x)}{f_{i}(x)} z\right) .
$$

Since on $U_{i} \cap U_{j}$ both $f_{i}$ and $f_{j}$ have $H \cap U_{i} \cap U_{j}$ as their zeroes, $f_{j} / f_{i}$ is a nowhere zero regular function on $U_{i} \cap U_{j}$. Thus the $g_{i j}$ define a line bundle $\mathcal{O}(H)$. To get the section $s$, over $U_{0}$ choose $s: x \mapsto(x, 1)$. Over $U_{i}$ this becomes $s: x \mapsto g_{0 i}(x, 1)=\left(x, f_{i}(x)\right)$, which is regular, and clearly $(s=0)=H$.

If $X=\mathbf{C P}^{n}$ and $H$ is a hyperplane then $\mathcal{O}(H) \cong \mathcal{O}(1)$.

(v) TANGENT BUNDLE. Let $V \subset \mathbf{C}^{n}$ be a smooth subvariety of dimension $k$. For each $x \in V$, there is a tangent $k$-plane to $V$ at $x$. If we translate this by the vector $-x$, then it becomes a vector subspace of $\mathbf{C}^{n}$. This gives us a rank $k$ subbundle of $V \times \mathbf{C}^{n}$, called the tangent bundle of $V$ and denoted by $T_{V}$. It has an intrinsic description as well. The tangent $k$-plane at $x$ is the vector space of derivations on holomorphic functions near $x$. If $z_{i}$ is a local coordinate system, then the operators $\sum a_{i} \partial / \partial z_{i}$ form this $k$-plane. Therefore $T_{V}$ is independent of the imbedding.

It $W \subset \mathbf{C P}^{n}$ is a smooth variety, then it can be covered by affine pieces $V_{i}=W \cap U_{i}$, and the $T_{V_{i}}$ patch together in the natural way to give $T_{W}$, the tangent bundle of $W$. Its local sections are of the form $\sum f_{i}(\mathbf{z}) \partial / \partial z_{i}$.

Its dual is called $\Omega_{W}^{1}$; its local sections are 1 -forms $\sum f_{i}(\mathbf{z}) d z_{i}$. Of special interest is its determinant bundle $\Omega_{W}^{k}=\operatorname{det} \Omega_{W}^{1}=\Lambda^{k} \Omega^{1}$ for $k=\operatorname{dim} W$. It is a line bundle whose local sections are $k$-forms: $f(\mathbf{z}) d z_{1} \wedge \cdots \wedge d z_{k} . \Omega_{W}^{k}$ is also 
called the canonical bundle of $W$, and is frequently denoted by $K_{W}$. It will be of great importance for us.

(vi) If $V \subset W$ are smooth varieties, then we have a natural inclusion $T_{V} \rightarrow T_{W} \mid V$. The quotient is again a vector bundle; at each $x \in V$ this quotient is the vector space of directions in the tangent space of $W$ that are "perpendicular" to the tangent space of $V$ at $x$. If $V(\varepsilon) \subset W$ is a tubular neighborhood of $V$, then one can think of this quotient as a linearized version of $V(\varepsilon)$. It is called the normal bundle and is denoted by $N_{V \mid W}$. Thus one has an exact sequence

$$
0 \rightarrow T_{V} \rightarrow T_{W} \mid V \rightarrow N_{V \mid W} \rightarrow 0 .
$$

Taking determinants we get

$$
K_{V} \cong K_{W} \mid V \otimes \operatorname{det} N_{V \mid W}
$$

Because of their importance in connection with maps, we study global sections a bit more. The fundamental result is the following

7.7. THEOREM. Let $p: V \rightarrow X$ be a vector bundle over a projective variety. Then $\operatorname{dim} \Gamma(X, V)$ is finite.

Proof. The sections are certain maps from $X$ to $V$. A slight change of 5.21 shows that every analytic section is algebraic. Therefore it does not matter which we consider. It is easier to work with the analytic case.

Fix some reasonable measure $d \mu$ on $X$ and a positive definite Hermitian form $h_{x}($,$) on each p^{-1}(x)$ such that these forms vary $C^{\infty}$ with $x$. For $s_{i} \in \Gamma(X, V)$ we define

$$
\left(s_{1}, s_{2}\right)=\int_{X} h_{x}\left(s_{1}(x), s_{2}(x)\right) d \mu .
$$

This defines an inner product on $\Gamma(X, V)$.

It is known classically that if $\left\{f_{i}\right\}$ is an $L^{2}$ bounded sequence of analytic functions on the unit disc, then there is a subsequence that converges on compact subsets to an analytic function. One can apply this to a covering of $X$ to conclude that $\Gamma(X, V)$ becomes a Hilbert space whose unit ball is compact. This obviously implies that it is finite dimensional.

7.8. ExAmples. (i) Let $\mathcal{O}_{X}$ be the trivial bundle $X \times \mathbf{C}$ on a connected projective variety. Then sections of $\mathcal{O}_{X}$ correspond to regular maps $X \rightarrow \mathrm{C}$, and therefore, by 5.11 , they are constants. Thus $\Gamma\left(X, \mathcal{O}_{X}\right) \cong \mathbf{C}$.

(ii) Let $L$ be a line bundle on $X$ with a nowhere zero section $s: X \rightarrow L$. We can define $f: X \times \mathbf{C} \rightarrow L$ by $f(x, z)=z s(x)$. This shows that $L$ is isomorphic to the trivial bundle.

(iii) One has a natural bilinear map $\Gamma\left(X, V_{1}\right) \otimes \Gamma\left(X, V_{2}\right) \rightarrow \Gamma\left(X, V_{1} \otimes V_{2}\right)$ given by $\left(s_{1} \otimes s_{2}\right)(x)=s_{1}(x) \otimes s_{2}(x)$.

(iv) COROLlary. Let $X$ be a projective variety; L a line bundle on $X ; L^{-1}$ its dual. If both $L$ and $L^{-1}$ have nontrivial sections, then $L \cong \mathcal{O}_{X}$.

Proof. Let $s$, resp. $t$, be a nontrivial section of $L$, resp. $L^{-1}$. Then $s \otimes t$ is a section of $L \otimes L^{-1} \cong \mathcal{O}_{X}$. By (i) this is a nonzero constant, and hence $s(x)$ is never zero. Now (ii) implies the claim. 
(v) Let $L$ be a line bundle and $s$ a nontrivial section. How can one get other sections of $L$ ? Assume that $t$ is another section. Then $f=t / s$ is a rational function on $X$. Conversely, if $t=f \cdot s$, then $t$ is a section of $L$, but maybe with some poles at the poles of $f$. The zeros of $s$ should cancel out the poles of $f$. This can be used for computations as follows.

(vi) Computing $\Gamma\left(\mathbf{C P}^{n}, \mathcal{O}(k)\right)$.

Recall that $\mathcal{O}(1)$ was given as the quotient of $\mathbf{C P}^{n} \times \mathbf{C}^{n+1}$ such that at $\left(x_{0}: \ldots: x_{n}\right)$ the quotient map is $\left(z_{0}, \ldots, z_{n}\right) \rightarrow \sum x_{i} z_{i} \in \mathbf{C} .(1,0, \ldots, 0)$ gives a section $s$ of $\mathcal{O}(1)$ which vanishes for those points with $x_{0}=0$. This is the hyperplane at infinity. Any other section is of the form $f \cdot s$, where $f$ is regular on $\mathbf{C}^{n} \cong U_{0}$ and has at most a simple pole at infinity. These are exactly the linear functions $f=\sum a_{i} x_{i} / x_{0}$. Hence the sections of $\mathcal{O}(1)$ can be identified with linear polynomials $\sum a_{i} x_{i}$. In particular, $\operatorname{dim} \Gamma\left(\mathbf{C P}{ }^{n}, \mathcal{O}(1)\right)=n+1$.

In $\mathcal{O}(k)$ we choose the basic section $s \otimes \cdots \otimes s$ ( $k$ times). Now we are looking for regular functions on $\mathbf{C}^{n} \cong U_{0}$ that have at most $k$-fold poles at infinity. As above we find that these correspond to degree $k$ homogeneous polynomials in $x_{0}, \ldots, x_{n}$. Thus

$$
\operatorname{dim} \Gamma\left(\mathbf{C P}^{n}, \mathcal{O}(k)\right)=\left(\begin{array}{c}
n+k \\
n
\end{array}\right) .
$$

If $k<0$, then by (iv) we have no sections.

(vii) Line bundles on curves. Let $C$ be a curve of genus $g$ and $L$ a line bundle over $C$ with a section $s$. Other sections of $L$ are given by $f \cdot s$, where $f$ is meromorphic on $C$ with poles only at the zeroes of $s$. The dimension of the space of such functions was computed in 3.8 and thus we get

$$
\operatorname{dim} \Gamma(C, L) \geqslant \#(\text { zeroes of } s)+1-g(C) .
$$

7.9. ThE IMPORTANCE OF $K_{X}$. Let $L$ be a line bundle on a projective variety. Let $s_{0}, \ldots, s_{n}$ be a basis of $\Gamma(X, L)$. Then this defines a map of $X$ into $\mathbf{C P}^{n}$. If one picks a different basis $s_{0}^{\prime}, \ldots, s_{n}^{\prime}$, then we get a different map. However the two differ only by a linear change of coordinates in $\mathbf{C P}^{n}$. Therefore $L$ itself determines an essentially unique map into $\mathbf{C P}^{n}$.

We still have $L$ as an arbitrary choice. Which line bundle should one pick on $X$ ? The advantage of $K_{X}$ is that it is "God-given"; it does not represent a new choice. Similarly, its tensor powers $K_{X}^{\otimes m}$ are distinguished. The choice of $m$ is something one can pick once and forever for all varieties.

Why do we concentrate on $K_{X}$ and not on $K_{X}^{-1}$ ? We will see (8.15 and 8.17) that $\Gamma\left(X, K_{X}^{\otimes m}\right)$ has very nice functoriality properties whereas $\Gamma\left(X, K_{X}^{-1}\right)$ does not.

There is only one remaining problem. $K_{X}^{\otimes m}$ might have very few sections, maybe only the trivial one. In this case we don't get any interesting map. Therefore we shall look into the structure of $K_{X}$ in detail and try to get information about those varieties for which $K_{X}^{\otimes m}$ does not give interesting maps.

Maybe the easiest way to extract information from $K_{X}^{\otimes m}$ is by counting the number of sections. $\operatorname{dim} \Gamma\left(X, K_{X}^{\otimes m}\right)$ is denoted by $P_{m}(X)$ and is called the $m$-genus of $X . P_{1}(X)$ is also denoted by $p_{g}(X)$ and is called the geometric genus. 
Next we compute $K_{X}$ in some cases.

7.10. EXAMPLES. (i) $K_{\mathbf{C P}^{n}} \cong \mathcal{O}(-n-1)$.

Proof. Let $\left(x_{0}: \ldots: x_{n}\right)$ be coordinates on $\mathbf{C P}^{n}$. On $U_{0}$ we pick coordinates $z_{i}=x_{i} / x_{0}$. Since $U_{0} \cong \mathbf{C}^{n}, K_{U_{0}}$ is trivial and the $n$-form $d z_{1} \wedge \cdots \wedge d z_{n}$ is a nowhere zero section. What happens at $\mathbf{C P}^{n}-U_{0}$ ? We pick, for instance, $U_{n}$ with coordinates $y_{j}=x_{j} / x_{n}$. Then $z_{i}=x_{i} / x_{0}=y_{i} / y_{0}$ for $i=1, \ldots, n-1$ and $z_{n}=1 / y_{0}$. Therefore

$d z_{1} \wedge \cdots \wedge d z_{n}=d \frac{y_{1}}{y_{0}} \wedge \cdots \wedge d \frac{y_{n-1}}{y_{0}} \wedge d \frac{1}{y_{0}}=(-1)^{n} \frac{1}{y_{0}^{n+1}} d y_{0} \wedge \cdots \wedge d y_{n-1}$.

Thus $d z_{1} \wedge \cdots \wedge d z_{n}$ is a rational section of $K_{\mathbf{C P}^{n}}$ which has an $(n+1)$-fold pole along the hyperplane $x_{0}=0$. Therefore $K_{\mathbf{C P}^{n}} \otimes \mathcal{O}(n+1)$ has a section

$$
\left(d z_{1} \wedge \cdots \wedge d z_{n}\right) \otimes\left(s^{n+1}\right) \text { which is nowhere zero. }
$$

This is therefore the trivial bundle, and hence $K_{\mathbf{C P}^{n}} \cong \mathcal{O}(-n-1)$.

(ii) $K_{\mathbf{C}^{n} / L} \cong \mathcal{O}_{\mathbf{C}^{n} / L}$ where $\mathbf{C}^{n} / L$ is as in 4.7.

PROof. Let $z_{1}, \ldots, z_{n}$ be coordinates on $\mathbf{C}^{n}$. Then $d z_{1} \wedge \cdots \wedge d z_{n}$ is a nowhere zero section of $K_{\mathbf{C}^{n}}$. This section is $L$-invariant, so it gives a nowhere zero section of $K_{\mathbf{C}^{n} / L}$.

(iii) Let $X$ and $Y$ be smooth projective varieties and let $p: X \times Y \rightarrow X$, $q: X \times Y \rightarrow Y$ be the coordinate projections. Then $K_{X \times Y} \cong p^{*} K_{X} \otimes q^{*} K_{Y}$.

PROOF. At each point of $X \times Y$, the tangent space decomposes into vertical and horizontal tangent vectors. Thus $T_{X \times Y} \cong p^{*} T_{X}+q^{*} T_{Y}$. Taking the determinant and dualizing gives our formula.

In order to get more examples we need the following

7.11. Proposition. Let $V$ be a vector bundle on a smooth variety $X$. Let $s: X \rightarrow V$ be a section and $Y \subset X$ be its set of zeros. Assume that $s(X)$ intersects the zero section transversally. Then $N_{Y \mid X} \cong V \mid Y$.

Proof. Let $Y(\varepsilon)$ be a small tubular neighborhood of $Y$ in $X$. It is intuitively clear that there is a retraction map $r: Y(\varepsilon) \rightarrow Y$. Unfortunately sometimes this $r$ cannot be chosen to be analytic. Still, assume that we have an analytic retraction. This will then extend to a retraction map $R: V|Y(\varepsilon) \rightarrow V| Y$.

Now if $x \in Y(\varepsilon)$, then the rule $x \rightarrow R(s(x))$ maps $Y(\varepsilon)$ onto a small neighborhood of the zero section of $V \mid Y$. Essentially by definition, $N_{Y \mid X}$ is the linearization of $Y(\varepsilon)$. This gives the required isomorphism.

In general one can still find $r$ and $R$ that are analytic up to first order along $Y$, and this is enough to conclude the proof.

7.12. Corollary. Let $H \subset \mathbf{C P}^{n}$ be a degree $k$ smooth hypersurface. Then $K_{H}=\mathcal{O}(k-n-1) \mid H$.

Proof. $H$ is given by a degree $k$ polynomial which is a section of $\mathcal{O}(k)$. Therefore $N_{H \mid \mathbf{C P}^{n}} \cong \mathcal{O}(k) \mid H$. By 7.5(vi), $K_{H} \cong K_{\mathbf{C P}^{n}} \mid H \otimes N_{H \mid \mathbf{C P}^{n}}$. 7.10(i) computes $K_{\mathbf{C P}^{n}}$, and this gives the result.

7.13. Definition. (i) Let $L$ be a line bundle with a section $s: X \rightarrow L$. Let $V(s) \subset X$ be the zero set. It is an algebraic variety of codimension one. By 6.1 this has a homology class $[V(s)] \in H_{2 n-2}(X, \mathbf{Z})(n=\operatorname{dim} X)$. If $s^{\prime}$ is another 
section then $f=s^{\prime} / s$ is a rational function with zero set $V\left(s^{\prime}\right)$ and polar set $V(s)$. Therefore, by $6.2,\left[V\left(s^{\prime}\right)\right]=[V(s)]$, and we have a well-defined class $[L] \in H_{2 n-2}(X, \mathbf{Z})$.

(ii) If $L$ has no regular section, then we take a meromorphic section $t$ with zero set $Z(t)$ and polar set $P(t)$ and we define $[L]=[Z(t)]-[P(t)]$.

(iii) If $X$ is smooth and projective, then $H_{2 n-2}$ can be naturally identified with $H^{2}$. In general we have only a map from $H^{2} \rightarrow H_{2 n-2}$. One can see that [L] can be lifted to $H^{2}(X, \mathbf{Z})$. We shall use it only for the smooth case. The resulting class in $H^{2}(X, \mathbf{Z})$ is denoted by $c_{1}(L)$ and is called the first Chern class of $L$.

From the definition it is clear that $c_{1}\left(\mathcal{O}_{X}\right)=0$. From 7.8(iii) we conclude that $c_{1}\left(L_{1} \otimes L_{2}\right)=c_{1}\left(L_{1}\right)+c_{1}\left(L_{2}\right)$.

(iv) If $V$ is a vector bundle over $X$ then we define $c_{1}(V)=c_{1}(\operatorname{det} V)$.

(v) If $C$ is a smooth projective curve, then $H^{2}(C, \mathbf{Z}) \cong \mathbf{Z}$. In this case, $c_{1}(L)$ can be considered as an integer. It is also called the degree of $L$ and is denoted by $\operatorname{deg} L$.

(vi) With the above definition and using the remark 7.8 (vii), we can put Riemann's theorem 3.8 in its more customary form:

RIEMANN - ROCH FOR CURVES. Let $L$ be a line bundle on a smooth projective curve $C$. Then

$$
\operatorname{dim} \Gamma(C, L) \geqslant \operatorname{deg} L+1-g
$$

and equality holds if $\operatorname{deg} L \geqslant 2 g-1$.

(vii) Let $X$ be a variety and $C \subset X$ be a projective curve. Then $[C] \in$ $H_{2}(X, \mathbf{Z})$ and we have a bilinear pairing $H_{2}(X, \mathbf{Z}) \otimes H^{2}(X, \mathbf{Z}) \rightarrow \mathbf{Z}$ to give intersection numbers $[C] \cdot c_{1}(L) \in \mathbf{Z}$ for any line bundle.

It can be directly obtained as follows. Let $g: \bar{C} \rightarrow C$ be the normalization of $C$. Then $g^{*} L$ is a line bundle on $\bar{C}$. Hence by (v) it has a degree. It is quite clear that $\operatorname{deg} g * L=[C] \cdot c_{1}(L)$.

(viii) Assume that $L$ is generated by sections (i.e., for every $x \in X$ there is a section $s$ of $L$ such that $s(x) \neq 0)$. Let $x \in C$ be a point and $s$ a section not zero at $x$. Then $g^{*} s$ is a nontrivial section of $g^{*} L$ and $\operatorname{deg} g^{*} L \geqslant 0$.

Now let $X \subset \mathbf{C P}{ }^{n}$ and consider $\mathcal{O}(1) \mid X$. From 7.8(vi) we see that $c_{1}(\mathcal{O}(1))$ $=\left[\right.$ hyperplane]. Therefore $[C] \cdot c_{1}(\mathcal{O}(1) \mid X)=[C] \cdot[$ hyperplane] $>0$ as we saw in the proof of 6.5 . This gives us

7.14. Corollary. Let $X$ be a projective variety and $L$ a line bundle that gives an embedding of $X$ into some projective space. Then $[C] \cdot c_{1}(L)>0$ for every curve; i.e., the linear function given by $L$ on $N E(X)$ is positive.

This comes very close to giving a characterization of such line bundles. The converse is the following result, which we cannot prove here.

7.15. KLEIMAN'S CRITERION. Let $L$ be a line bundle on a projective variety $X$. The following two statements are equivalent.

(i) $L^{\otimes m}$ gives an embedding into some projective space for all sufficiently large $m$.

(ii) The linear function given by $L$ on $\overline{N E}(X)-\{0\}$ is positive. 
7.16. Definitions. (i) A line bundle $L$ satisfying the above conditions is called ample.

(ii) A line bundle $L$ is called nef if the linear function given by $L$ on $\overline{N E}(X)$ is nonnegative. ("nef" comes from the very misleading terminology "numerically effective".) Equivalently, $L$ is nef if $[C] c_{1}(L) \geqslant 0$ for every curve $C \subset X$. This condition is much easier to check than ampleness because we do not have to consider limits of curves.

Finally we give some further examples of line bundles.

7.17. TheOREM. Every line bundle on $\mathrm{CP}^{n}$ is isomorphic to $\mathcal{O}(m)$ for some $m \in \mathbf{Z}$.

Proof. We start with $\mathbf{C P}^{1}$ and a line bundle $L$ of degree $m$. Then $L^{\prime}=L \otimes \mathcal{O}(-m)$ is of degree 0 . Let $s$ be a meromorphic section of $L^{\prime}$; it has the same number of zeroes and poles. Thus multiplying $s$ by functions of the form $(z-a) /(z-b)$, we can get rid of all the poles and zeroes and get a nowhere vanishing section of $L^{\prime}$. Hence, by 7.8(ii), $L^{\prime} \cong \mathcal{O}$ and so $L \cong \mathcal{O}(m)$.

Now let $L$ be a line bundle on $\mathbf{C P}^{n}$ and let $C \subset \mathbf{C P}^{n}$ be a line. Let $m=[C] c_{1}(L)$. Then taking $L^{\prime}=L \otimes \mathcal{O}(-m)$, we get $[C] c_{1}\left(L^{\prime}\right)=0$. Hence by the above consideration, $L^{\prime} \mid C \cong \mathcal{O}_{C}$.

If we fix $p \in \mathbf{C P}$, then lines through $p$ cover $\mathbf{C P}^{n}$. If we fix a $0 \neq l \in L^{\prime} \mid p$, then for each line $C$ containing $p$ there is a unique section $s_{C}: C \rightarrow L^{\prime} \mid C$ such that $s_{C}(p)=l$. These sections glue together to a nowhere zero section of $L^{\prime}$. Hence $L^{\prime} \cong \mathcal{O}_{\mathbf{C P}^{n}}$ and we are done.

Now we can easily prove the result promised in 2.30 .

\subsection{Corollary. Aut $\mathrm{CP}^{n} \cong \operatorname{PGL}(n+1, \mathrm{C})$.}

Proof. Let $f: \mathbf{C P}^{n} \rightarrow \mathbf{C P}^{n}$ be an automorphism. We want to determine $f^{*} \mathcal{O}(1)$. Every line bundle on $\mathbf{C P}^{n}$ is a tensor power of $\mathcal{O}(1)$; thus the same holds for $f^{*} \mathcal{O}(1)$. Hence it is either $\mathcal{O}(1)$ or $\mathcal{O}(-1)$. $\mathcal{O}(1)$ has several sections and so does $f^{*} \mathcal{O}(1)$ and thus necessarily $f^{*} \mathcal{O}(1) \cong \mathcal{O}(1)$. Therefore $f$ gives a map $f^{*}: \Gamma\left(\mathbf{C P}{ }^{n}, \mathcal{O}(1)\right) \rightarrow \Gamma\left(\mathbf{C P}{ }^{n}, \mathcal{O}(1)\right)$. Now $\Gamma\left(\mathbf{C P}{ }^{n}, \mathcal{O}(1)\right)$ is exactly the dual of the $\mathbf{C}^{n+1}$ out of which $\mathbf{C P}^{n}$ was made, and so the above $f^{*}$ gives us an element of $\operatorname{Aut}\left(\mathbf{C}^{n+1}\right)$. This induces an automorphism of $\mathbf{C P}^{n}$ which is easily seen to be $f$.

7.19. REMARK. One should not think that in general $c_{1}(L)$ determines $L$. As an example, let $C$ be any curve of genus at least one. Let $p, q \in C$ be different points. Then $c_{1}(\mathcal{O}(p))=c_{1}(\mathcal{O}(q))$, but $\mathcal{O}(p) \not \mathcal{O}(q)$. Assume the contrary. Then $\mathcal{O}(p) \otimes \mathcal{O}(q)^{-1} \cong \mathcal{O}$ has a nowhere zero section $s$. On the other hand, from the construction (7.6(iv)), it has a section $s^{\prime}$ with a simple zero at $p$ and a simple pole at $q$. Then $s^{\prime} / s$ is a function on $C$ with a single simple pole. As in 3.10 this implies that $C \cong \mathbf{C P}^{1}$, a contradiction.

\section{How to understand algebraic varieties.}

8.1. Two APPROACHES. There are two basic approaches to the geometry of algebraic varieties. One stems from a very general point of view: one would like to have a broad understanding of all algebraic varieties. Therefore, one searches for general structure theorems. The other point of view is that special 
frequently occurring varieties are the most interesting and we should get detailed information about them.

Let me illustrate this difference by an example. Assume that we are given a particular three-dimensional algebraic variety $X$, and we can prove that it is isomorphic to a smooth hypersurface of degree five in $\mathbf{C P}^{4}$. This makes an adherent of the general point of view perfectly happy. A proponent of the second approach would, however, politely point out that he is interested only in hypersurfaces anyhow and that he is busy understanding maps from $\mathbf{C P}^{1}$ to quintic hypersurfaces in $\mathbf{C P}^{4}$. This latter turns out to be a very interesting and subtle question.

I will discuss solely the general structural approach, a choice dictated by personal taste.

8.2. BASIC STRATEGY. These ideas are not new; they go back to the Italian school of the beginning of the century.

Step one is to define an equivalence relation on all varieties. Two varieties will be declared equivalent if they look the same almost everywhere. Then we try to understand these equivalence classes by proving that certain simple surgery operations allow us to get from one member to another.

Step two is to find ways of associating a single variety to a given equivalence class. This frequently reduces the problem of understanding different equivalence classes to understanding certain individual varieties.

Step three is then to use the special properties of these individual varieties to get an overview of all equivalence classes.

This approach was developed, and is spectacularly successful, for algebraic surfaces: two-dimensional projective varieties. In a quite miraculous manner, the complexity of algebraic surfaces comes either from step one, or from step three, but not both. More explicitly, one of the following alternatives takes place.

(i) The equivalence class contains a very simple member (like $\mathbf{C} \mathbf{P}^{2}$ ). In this case the relationship of surfaces within the class is very complicated.

(ii) The equivalence class contains only complicated surfaces, but they are related to each other in a simple way.

Considerable advances were made recently concerning three-dimensional varieties. Although everything is much more complicated, a similar pattern seems to emerge.

Now we look into the above program in more detail. First we define the equivalence relation.

8.3. Definition. (i) A map $f: X \rightarrow Y$ is called birational if there are proper closed subsets $V \subset X$ and $Z \subset Y$ such that $f: X-V \rightarrow Y-Z$ is an isomorphism. In this case, $f$ has an inverse $f^{-1}: Y \rightarrow X$ which is a rational map, regular on $Y-Z$.

(ii) Two irreducible projective varieties are said to be birationally equivalent or simply birational if there is a birational map between them. This is clearly an equivalence relation.

(iii) Similarly, one can define bimeromorphic maps between complex manifolds.

(iv) Two varieties $X$ and $Y$ are called isomorphic if there are everywhere 
regular maps $f: X \rightarrow Y$ and $g: Y \rightarrow X$ such that $f \circ g=$ id and $g \circ f=$ id. I usually will not distinguish between isomorphic varieties.

8.4. ExAMPLE. (i) Let $Y$ be a variety and let $Z$ be a closed subvariety. Then the inclusion map $i: Y-Z \rightarrow Y$ is a birational isomorphism.

(ii) The map from $\mathbf{C P}^{2} \rightarrow \mathbf{C P}^{1} \times \mathbf{C P}^{1}$ given by $(x: y: z) \rightarrow((x: z),(y: z))$ is birational. It is not regular at $(0: 1: 0)$ and $(1: 0: 0)$. The line $z=0$ is mapped to a point $((1: 0),(1: 0))$.

(iii) In general $\mathbf{C P}^{n+m}$ is birational to $\mathbf{C P}^{n} \times \mathbf{C P}^{m}$ via $\mathbf{C P}^{n+m} \supset \mathbf{C}^{n+m} \cong$ $\mathbf{C}^{n} \times \mathbf{C}^{m} \subset \mathbf{C P}^{n} \times \mathbf{C P}^{m}$

(iv) Let $f: \mathbf{C} \rightarrow \mathbf{C}^{2}$ be given by $f(t)=\left(t^{n}, t^{m}\right)$, and let $V \subset \mathbf{C}^{2}$ be the image. $f: \mathbf{C} \rightarrow V$ is birational iff $(n, m)=1$. It is an isomorphism iff $n=1$ or $m=1$. The $n=2, m=3$ case was considered in detail in 5.3(iii).

(v) Let $Q \subset \mathbf{C} \mathbf{P}^{n+1}$ be a smooth hypersurface of degree two. Let $q \in Q$ be a point and let $H \subset \mathbf{C P}^{n+1}$ be a hyperplane not containing $q$. I define a map $p: Q \rightarrow H$ as follows. Let $q \neq q^{\prime} \in Q$. Connect $q$ and $q^{\prime}$ with a line, and let $p\left(q^{\prime}\right)$ be the intersection point of this line with $H$. One can easily see that this map is birational.

8.5. FUNDAMENTAL PROBlem ABOUT birational MAPS. Is there a set of "elementary" birational maps such that any birational map can be written as a composite of elementary maps?

This problem is satisfactorily settled in $\operatorname{dim} 2$ but it is very much open in $\operatorname{dim} 3$. It is not even clear which maps should be considered elementary. One candidate is the following:

8.6. EXAMPLE: BLowING UP. This quite simple process starts with a variety $X$ and creates another one, $X^{\prime}$, which is birationally equivalent to $X$.

(i) PoInT IN $\mathbf{C P}^{n}$. The idea is to replace a point of $\mathbf{C P}^{n}$ with the set of directions through that point, i.e., with a copy of $\mathbf{C} \mathbf{P}^{n-1}$. With actual equations it can be described as follows. Let $\left(y_{0}: \ldots: y_{n}\right)$ be coordinates on $\mathbf{C P}^{n}$, $\left(z_{0}: \ldots: z_{n-1}\right)$ those on $\mathbf{C P}{ }^{n-1}$. Let $B_{0} \mathbf{C P}^{n}$ be the subset of $\mathbf{C P}^{n} \times \mathbf{C P}^{n-1}$ defined by the equations $y_{i} z_{j}=z_{i} y_{j}, 0 \leqslant i, j \leqslant n-1$. Let $p: B_{0} \mathbf{C P}^{n} \rightarrow \mathbf{C P}^{n}$ be the first projection. If $x \in \mathbf{C P}^{n}$ is the point $(0: \ldots: 0: 1)$, then the equations give no conditions on the $y_{i}$ 's so $p^{-1}(0: \ldots: 0: 1) \cong \mathbf{C} \mathbf{P}^{n-1}$. If, say, $z_{0} \neq 0$, then $y_{i}=y_{0} \cdot z_{i} / z_{0}$ and hence $\left(y_{0}: \ldots: y_{n-1}\right)$ is uniquely determined. Thus $p$ is a birational map as we wanted.

(ii) LINEAR SUBSPACE OF $\mathbf{C P}^{n}$. If $L \subset \mathbf{C P}^{n}$ is a linear subspace, then we can try to replace $L$ by the set of directions of lines "perpendicular" to $L$. Working as above, let (y) be coordinates on $\mathbf{C P}{ }^{n},(\mathbf{z})$ be those on $\mathbf{C P}^{k}$, and let $B_{L} \mathbf{C P}{ }^{n}$ be the subset of $\mathbf{C P}^{n} \times \mathbf{C P}^{k}$ given by $y_{i} z_{j}=z_{i} y_{j}$ for $0 \leqslant i, j \leqslant k$. The first projection $p: B_{L} \mathbf{C P} \mathbf{P}^{n} \rightarrow \mathbf{C P}^{n}$ is again birational; $p^{-1}(x) \cong \mathbf{C P}^{k}$ if $x \in L=$ ( $\left.y_{0}=\cdots=y_{k}=0\right)$ and a single point otherwise.

It is easy to check that $B_{L} \mathbf{C P}^{n}$ is smooth.

(iii) SMOOTH SUBVARIETY, ANALYTIC CASE. Let $X$ be a smooth variety (or complex manifold) and let $Y \subset X$ be a smooth subvariety. We want to define $B_{Y} X$. Each point $x \in Y \subset X$ has a small neighborhood in the form of an open ball $D \subset \mathbf{C}^{n}$ and $Y \cap D$ is given by $y_{1}=\cdots=y_{k}=0$. We define $B_{Y \cap D} D$ to be the subset of $D \times \mathbf{C P} \mathbf{P}^{k-1}$ given by the equations $y_{i} z_{j}=z_{i} y_{j}$ for $1 \leqslant i, j$ $\leqslant k$, where for convenience we pick $\left(z_{1}: \ldots: z_{k}\right)$ as coordinates on $\mathbf{C P}^{k-1}$. 
Again one can see that $B_{Y \cap D} D$ is smooth for all $D$ and they patch together to give $p: B_{Y} X \rightarrow X$, a bimeromorphic map. One can actually see that $B_{Y} X$ is projective if $X$ is.

Blowing-ups go a long way toward simplifying rational maps. This is the content of the following basic and very hard theorem of Hironaka:

8.7. ELIMINATION OF INDETERMINACIES. Let $X$ be a smooth projective variety and let $f: X \rightarrow Z$ be a rational map. Then there is a finite sequence of blowing-ups

$$
g_{n}: X_{n} \rightarrow X_{n-1} \rightarrow \cdots \rightarrow X_{1} \rightarrow X_{0}=X
$$

such that $f \circ g_{n}$ is everywhere regular.

8.8. REMARK. (i) For $\operatorname{dim} X=2$ we will prove this in 9.7.

(ii) In the spirit of 8.5 , this means that "elementary maps" could mean inverses of blowing-ups and regular birational maps. This is a very useful result. However, in dim $\geqslant 3$ regular birational maps can be very complicated. Mori's program will provide an approach to their structure.

There is another general result of Hironaka which further clarifies the concept of birational equivalence. This is a generalization of 3.12.

8.9. RESOLUTION OF SINGULARITIES. Let $X$ be a projective variety. Then there is a regular birational map $f: Y \rightarrow X$ where $Y$ is smooth and projective.

Any such $Y$ will be called a resolution of the singularities of $X$. This result indicates that from the point of view of birational geometry, it is sufficient to consider smooth varieties. This is certainly true, but unfortunately $X$ and $Y$ can be very different. Therefore this reduction to the smooth case is not very useful.

It is time to concentrate now on the similarities between birationally equivalent varieties. To this end, let us now recall 5.19: a rational map between smooth projective varieties is regular outside a codimension two set. This has several consequences for birationally equivalent varieties.

8.10. TheOREM. Let $f: C \rightarrow C^{\prime}$ be a birational map between smooth projective curves. Then $f$ is an isomorphism.

Proof. Since $\operatorname{dim} C=1$, the codimension-two exceptional set is empty. Thus $f$ is regular and so is $f^{-1}$. 8.4(ii) shows that the corresponding statement is false for surfaces.

8.11. TheOREM. Let $X$ and $X^{\prime}$ be birational smooth projective varieties. Then the fundamental groups $\pi_{1}(X)$ and $\pi_{1}\left(X^{\prime}\right)$ are isomorphic.

Proof. Let $f: X \rightarrow X^{\prime}$ be a birational map, regular outside $Z \subset X$. $Z$ has complex codimension two, hence real codimension at least four. Therefore, $\pi_{1}(X) \cong \pi_{1}(X-Z)$. $f$ maps $X-Z$ into $X^{\prime}$, and this gives $\pi_{1}(X-Z) \rightarrow$ $\pi_{1}\left(X^{\prime}\right)$. So we have $f_{*}: \pi_{1}(X) \rightarrow \pi_{1}\left(X^{\prime}\right)$. It is clear that $\left(f^{-1}\right)_{*}$ gives the inverse to $f_{*}$.

The following results are crucial. 
8.12. Proposition. Let $g: U \rightarrow V$ be a regular map between smooth varieties of the same dimension $n$. Then there is a natural map $g^{*} K_{V} \rightarrow K_{U}$. If $u \in U$ then the map between the lines $g^{*} K_{V}\left|u \rightarrow K_{U}\right| u$ is an isomorphism iff $g$ is a local diffeomorphism near $u$.

Proof. Let $\left(u_{i}\right)$ be local coordinates at $u$ and $\left(v_{j}\right)$ be local coordinates at $v=g(u)$. Let $g^{*}\left(v_{j}\right)=g_{j}\left(u_{1}, \ldots, u_{n}\right)$. The line of $K_{V}$ at $v$ is given by the vectors $c d v_{1} \wedge \cdots \wedge d v_{n}(c \in \mathbf{C})$. The usual pull-back map is $g^{*}\left(d v_{j}\right)=$ $d g^{*}\left(v_{j}\right)=\sum \partial g_{j} / \partial u_{i} \cdot d u_{i}$; thus the map $g^{*} K_{V} \rightarrow K_{U}$ is given by $c d v_{1} \wedge \cdots \wedge d v_{n} \mapsto c g^{*} d v_{1} \wedge \cdots \wedge g^{*} d v_{n}=c \operatorname{det}\left(\partial g_{j} / \partial u_{i}\right) d u_{1} \wedge \cdots \wedge d u_{n}$. This map is an isomorphism iff $\operatorname{det}\left(\partial g_{j} / \partial u_{i}\right) \neq 0$. By the implicit function theorem, the latter is equivalent to $g$ being a local diffeomorphism.

8.13. Corollary. Let $E \subset U$ be the subset where $g$ is not a local diffeomorphism. Then any irreducible component of $E$ has dimension $n-1$ unless $E=U$.

Proof. Let $u \in E$. Then near $u, E$ is defined by one equation: $\operatorname{det}\left(\partial g_{j} / \partial u_{i}\right)$ $=0$. By 2.27 this implies $\operatorname{dim} E \geqslant n-1$ unless $\operatorname{det}\left(\partial g_{j} / \partial u_{i}\right) \equiv 0$.

8.14. Corollary. Let $C \subset U$ be an irreducible compact curve such that $C \not \subset E$. Then $[C] c_{1}\left(K_{U}\right) \geqslant[g(C)] c_{1}\left(K_{V}\right)$. Equality holds iff $C \cap E=\varnothing$.

Proof. Assume for simplicity that $K_{V}$ has a global section $\omega$ with zero set $Z \subset V$. Then $g^{*} \omega$ is a section of $g^{*} K_{V}$ with zero set $g^{-1}(Z)$. This gives a section of $K_{U}$ which will vanish in addition along $E=\bigcup E_{k}$. Since multiplicities have to be taken into account this gives

$$
c_{1}\left(K_{U}\right)=\left[g^{-1}(Z)\right]+\sum a_{k}\left[E_{k}\right] \quad\left(a_{k}>0\right) .
$$

Now $[C]\left[g^{-1}(Z)\right]=[g(C)][Z]$ and $[C] \cdot \sum a_{k}\left[E_{k}\right] \geqslant 0$ with equality iff $C \cap E$ $=\varnothing$.

8.15. ThEOREM. Let $f: X \rightarrow Y$ be a rational map between smooth projective varieties of the same dimension $n$. Assume that $f(X)$ is not contained in any proper closed subset of $Y$. Then $f$ induces an injection

$$
f^{*}: \Gamma\left(Y, K_{Y}^{\otimes m}\right) \rightarrow \Gamma\left(X, K_{X}^{\otimes m}\right) \text {. }
$$

If $f$ is birational then $f^{*}$ is an isomorphism. Thus $P_{m}(Y)=P_{m}(X)$ for every $m \geqslant 1$.

Proof. Let $Z \subset X$ be the set where $f$ is not defined. By $5.19 \operatorname{dim} Z \leqslant$ $\operatorname{dim} X-2$. Let $U=X-Z, V=Y, g=f \mid U$. From 8.12 we get a map $g^{*} K_{V}^{\otimes m} \rightarrow K_{U}^{\otimes m}$ and thus maps

$$
\Gamma\left(Y, K_{Y}^{\otimes m}\right) \rightarrow \Gamma\left(U, g^{*} K_{V}^{\otimes m}\right) \rightarrow \Gamma\left(U, K_{U}^{\otimes m}\right) .
$$

By $7.5 \Gamma\left(U, K_{U}^{\otimes m}\right)$ injects into $\Gamma\left(X, K_{X}^{\otimes m}\right)$ and this gives $f^{*}$. If $f$ is birational then $\left(f^{-1}\right)^{*}$ gives the inverse of $f^{*}$.

The previous theorem leads us directly to step two of the main program.

8.16. Definition. Let $X$ be a smooth projective variety. Let $\phi_{m}: X \rightarrow \mathbf{C P}^{\text {? }}$ be the map given by $\Gamma\left(X, K_{X}^{\otimes m}\right)$; it is called the $m$-canonical map. The closure of the image is a projective variety called the $m$-canonical image. It is denoted by $X^{[m]}$. 
8.17. CoROllaRy. If $X$ and $X^{\prime}$ are smooth projective varieties which are birational to each other, then $X^{[m]}$ and $X^{\prime[m]}$ differ only by a linear automorphism of $\mathbf{C P}$ ?. In particular, they are isomorphic.

Proof. If $f: X \rightarrow X^{\prime}$ is birational, then we get an isomorphism $\Gamma\left(X^{\prime}, K_{X^{\prime}}^{\otimes m}\right) \stackrel{\sim}{\rightarrow} \Gamma\left(X, K_{X}^{\otimes m}\right)$. If we construct the $m$-canonical map using "the same" bases in these two vector spaces, then we clearly get that $X^{\prime m]}$ and $X^{[m]}$ are the same subsets of $\mathbf{C P}$ ?. This proves the corollary.

In this way, to each smoth projective variety $X$ we associate an infinite sequence of new varieties $X^{[1]}, X^{[2]}, \ldots$, and each of these depends only on the birational equivalence class of $X$. In general $X^{[n]}$ is not birational to $X$, so we might lose considerable information.

Experience shows that the $X^{[i]}$ can be very different from each other, but there is a "main series" of them. Namely one can show

8.18. Proposition. If $n$ and $m$ are sufficiently large and divisible, then $X^{[n]}$ and $X^{[m]}$ are birational to each other. Their birational equivalence class will be called-with slight abuse of language - the Iitaka variety $I(X)$, and the natural map $\phi: X \rightarrow I(X)$ the stable canonical map.

Of course this is not quite what we wanted. We would like to associate one variety, and not a birational equivalence class, to our original birational equivalence class. This leads to the following

8.19. Fundamental PROBLEM. Let $X$ be a smooth projective variety. Is it true that if $n$ and $m$ are sufficiently large and divisible, then $X^{[n]}$ and $X^{[m]}$ are actually isomorphic?

This is very easy for $\operatorname{dim} X=1$. If $X$ is a surface, then the proof is already nontrivial. One of the goals and major achievements of Mori's program is the affirmative answer to this problem for three-dimensional varieties.

8.20. EXAmples. (i) By 7.9(i), $K_{\mathbf{C P}^{n}}=\mathcal{O}(-n-1)$, and so

$$
K_{\mathbf{C P}^{n}}^{\otimes m}=\mathcal{O}(-n m-m) \text {. }
$$

Therefore $P_{m}\left(\mathbf{C P}^{n}\right)=0$ for all $m \geqslant 1$; hence $I\left(\mathbf{C P}^{n}\right)=\varnothing$.

(ii) By 7.10(ii), $K_{\mathbf{C}^{n} / L} \cong \mathcal{O}$, hence the same is true for all tensor powers. Since $\operatorname{dim} \Gamma(X, \mathcal{O})=1$, this gives $P_{m}\left(\mathbf{C}^{n} / L\right)=1$. Thus $I\left(\mathbf{C}^{n} / L\right)=$ point; again not much information is contained in it.

(iii) By 7.10(iii) $K_{X \times Y}^{\otimes m} \cong p^{*} K_{X}^{\otimes m} \otimes q^{*} K_{Y}^{\otimes m}$. If $s \in \Gamma\left(X, K_{X}^{\otimes m}\right)$ and $t \in$ $\Gamma\left(Y, K_{Y}^{\otimes m}\right)$ then $p^{*} s \otimes q^{*} t \in \Gamma\left(X \times Y, K_{X \times Y}^{\otimes m}\right)$. One can easily see that such sections span the space of all sections. Thus $P_{m}(X \times Y)=P_{m}(X) \cdot P_{m}(Y)$ and $I(X \times Y) \cong I(X) \times I(Y)$.

(iv) Let $H \subset \mathbf{C P}^{n}$ be a smooth hypersurface of degree $k$. Then by 7.11 $K_{H} \cong \mathcal{O}(k-n-1) \mid H$. There are three cases to consider.

(a) $k<n+1$. Then we get $k-n-1<0$, hence $P_{m}(H)=0$ for every $m$.

(b) $k=n+1$. Then $K_{H} \cong \mathcal{O}_{H}$, and thus $P_{m}(H)=1$.

(c) $k>n+1$. In this case $\mathcal{O}(m(k-n-1))$ has many sections over $\mathbf{C P}^{n}$, and some of them will not restrict to zero on $H$. In fact, one can compute that

$$
P_{m}(H)=\left(\begin{array}{c}
m(k-n-1)+n \\
n
\end{array}\right)-\left(\begin{array}{c}
m(k-n-1)+n-k \\
n
\end{array}\right) .
$$


(v) CoROllaRY. Let $H$ and $H^{\prime}$ be smooth hypersurfaces of degree $k$ and $k^{\prime}$ in $\mathbf{C P}^{n}$. Assume that $k \geqslant n+1$ and $k \neq k^{\prime}$. Then $H$ and $H^{\prime}$ are not birationally equivalent.

Proof. If $H$ and $H^{\prime}$ are birational, then $P_{m}(H)=P_{m}\left(H^{\prime}\right)$. We have formulas for these in terms of $k$ and $k^{\prime}$, and one can conclude the proof after some playing around.

(vi) What if both $k, k^{\prime}<n+1$ ? First of all, by $8.4(\mathrm{v})$ a smooth quadric $(k=2)$ is birational to a hyperplane $\left(k^{\prime}=1\right)$. A more sophisticated example is the following.

(vii) Consider the smooth cubic $C \subset \mathbf{C} \mathbf{P}^{2 n+1}$ given by the equation $x_{0} x_{1}^{2}+$ $x_{1} x_{2}^{2}+\cdots+x_{2 n+1} x_{0}^{2}=0$. Let $L_{1}$, resp. $L_{2}$, be the linear subspace given by equations $x_{0}=x_{2}=\cdots=x_{2 n}=0$, resp. $x_{1}=x_{3}=\cdots=x_{2 n+1}=0$. It is clear that $L_{i} \subset C$. Now look at the map $f: L_{1} \times L_{2} \rightarrow C$ defined as follows. Choose $x_{i} \in L_{i}$. Then connect $x_{1}$ and $x_{2}$ with a line in $\mathbf{C P}^{2 n+1}$. This line will intersect the cubic in three points; two of these points are $x_{1}, x_{2}$. Let $f\left(x_{1}, x_{2}\right)$ be the third point. With some work one can see that $f$ is indeed birational. Thus by 8.4 (iii) we see that $C$ is birationally equivalent to $\mathbf{C P}^{2 n}$.

(viii) In contrast, a smooth cubic in $\mathbf{C P}{ }^{4}$ is never birational to $\mathbf{C P}^{3}$, but this is hard to prove (Clemens-Griffiths).

8.21. ExAMPLE. Assume that $X$ is a smooth projective variety such that $K_{X}$ is ample. Then, by definition, for all sufficiently large $n, X \cong X^{[n]}$; so $X=I(X)$ and the answer to the fundamental problem is affirmative. This is the case for all smooth hypersurfaces $H \subset \mathbf{C P}^{n}$ of degree at least $n+2$.

The study of the stable canonical map gives a subdivision of all varieties into four classes. For convenience we introduce the notion of Kodaira dimension: $\kappa(X)=\operatorname{dim} I(X)$. We set $\kappa(X)=-\infty$ if $I(X)=\varnothing$.

8.22. BASIC SUbdivision. (i) $\kappa(X)=-\infty$; i.e., $P_{m}(X)=0$ for all $m$. Hypersurfaces of low degree are such examples.

(ii) $\kappa(X)=0$; i.e. $P_{m}(X) \leqslant 1$, with equality for some $m$. Examples are $\mathrm{C}^{n} / L$ and $H \subset \mathbf{C P}{ }^{n}$ of degree $n+1$.

(iii) $0<\kappa(X)<\operatorname{dim} X$. Then $\phi: X \rightarrow I(X)$ is a very interesting map. By some sort of induction on the dimension, we know more about $I(X)$ and about the fibers of $\phi$. Thus we hope to piece together information about lower dimensional-varieties to get some results about $X$ itself.

(iv) $\kappa(X)=\operatorname{dim} X$. These are called varieties of general type. This should be considered the "largest" class. In this case, in fact, $\phi: X \rightarrow I(X)$ is birational. Therefore, if the answer to the fundamental problem is yes, then we have completed step two of the basic strategy.

8.23. Once algebraic varieties are divided into these four classes, the investigation of individual classes should follow. Some program in this direction was outlined for the last two classes, i.e., for $\kappa(X)>0$. In the case of the first two classes, the stable canonical map offers little help, and we should turn to other methods. I think it is fair to say that at present very little is known about varieties with $\kappa(X)=0$; even a conjectural approach to their structure theory is lacking. The class $\kappa(X)=-\infty$ is, however, intimately tied up with maps from $\mathbf{C P}^{1}$ to $X$. We illustrate this with an example. 
8.24. EXAmple. Rational CURVES ON hypersurfaces. Let $H \subset \mathbf{C P}^{n}$ be a hypersurface given by an equation $h\left(x_{0}, \ldots, x_{n}\right)=0$ of degree $k$. Let $\mathbf{b} \in H$ be a point; we would like to find a map $f: \mathbf{C P}^{1} \rightarrow H$ such that $f(0: 1)=\mathbf{b}$. A map of $\mathbf{C P} \mathbf{P}^{1}$ into $\mathbf{C P} \mathbf{P}^{n}$ is given by $n+1$ homogeneous polynomials of a certain degree, say $m$. By passing to $\mathbf{C}$ instead, the map is given by

$$
t \rightarrow\left(\sum_{0}^{m} a_{i}^{0} t^{i}: \ldots: \sum_{0}^{m} a_{i}^{n} t^{i}\right) \in \mathbf{C P}^{n} .
$$

The condition $f\left(\mathbf{C P}^{1}\right) \subset H$ can be given by

$$
h \circ f=h\left(\sum_{0}^{m} a_{i}^{0} t^{i}, \ldots, \sum_{0}^{m} a_{i}^{n} t^{i}\right) \equiv 0 .
$$

Since $\operatorname{deg} h=k$, this can be viewed as a degree $m k$ polynomial in $t$ whose coefficients are polynomials in the $a_{j}^{i}$ 's. Thus we have altogether $m k+1$ equations for the $a_{j}^{i}$, s; each of these coefficients must be zero. How many free variables do we have? We want $f(0: 1)=\mathbf{b}$. Therefore $\left(a_{0}^{0}: \ldots: a_{0}^{n}\right)=$ $\left(b_{0}: \ldots: b_{n}\right)$, so we can as well pick $a_{0}^{i}=b_{i}$.

With this choice the degree zero coefficient of $h \circ f$ becomes zero, therefore we drop that equation. There is one more degree of internal freedom; the reparametrization $t=c t^{\prime}$ will change $a_{j}^{i}$ to $c^{j} a_{j}^{i}$. Therefore we can normalize, setting $a_{1}^{0}=1$. This leaves us with $m k$ equations in $(n+1) m-1$ variables. Therefore we expect that

(i) If $k<n+1$, then through every point of $H$ there is a rational curve.

(ii) If $k \geqslant n+1$, then there is no rational curve through a general point of $H$.

Though the above arguments are a bit shaky, the conclusions are indeed true.

Comparing this with 8.20(iii), we get that, for a smooth hypersurface $H$, $\kappa(H)=-\infty$ iff $H$ is covered by rational curves. This leads to a possible structural characterization of the class $\kappa=-\infty$ :

8.25. $\kappa=-\infty$ ChARACTERIZATION PROBLEM. Is it true that for a smooth projective variety $X, \kappa(X)=-\infty$ iff $X$ is covered by rational curves?

Assume that $X$ is covered by rational curves. One can see that most of $X$ is covered by a single family, i.e., there is a variety $Y(\operatorname{dim} Y=\operatorname{dim} X-1)$ and a dominant rational map $f: Y \times \mathbf{C P}^{1} \rightarrow X$. By 8.20 (iii) $P_{m}\left(Y \times \mathbf{C P}^{1}\right)=0$ and so by $8.15, P_{m}(X)=0$, and thus $\kappa(X)=-\infty$. Also note that one can get from 8.14 that $K_{X}$ is not nef. The hard part is the converse. It has been known to be true for curves and surfaces since the turn of the century, but a really conceptual proof was achieved only through Mori's program. The recent solution of this problem for threefolds demonstrates the power of the new methods.

8.26. Rational CURVES. The previous problem is only one of several examples that indicate that understanding rational curves lying on a given variety $V$ gives a key to understanding $X$. In general $X$ has several very nice properties if it contains no rational curves. The more rational curves $X$ contains the more complicated the birational geometry of $X$. Subsequent chapters abound with such examples. This is also one of the guiding principles of Mori's program. 
9. Birational geometry of surfaces. Surfaces provide the only large class of examples where step one of the basic strategy (8.2) is well understood. This will be discussed in the present chapter.

9.1. INTERSECTION THEORY. If $X$ is a smooth projective surface, then it is oriented by 6.3; thus Poincaré duality identifies $H_{2}$ and $H^{2}$. If $C_{i} \subset X$ is a curve, then $\left[C_{i}\right] \in H_{2}(X, \mathbf{Z})$ and one can talk about $\left[C_{1}\right] \cdot\left[C_{2}\right] \in H^{4}(X, \mathbf{Z}) \cong \mathbf{Z}$. In particular, the self-intersection $[C] \cdot[C]$ is defined.

If $f: X \rightarrow Y$ is a surjective map between surfaces, then Poincaré duality allows us to define $f_{*}$ and $f^{*}$ on either homology or cohomology groups. We shall freely use this.

I also recall the following basic computational tool: level sets of a meromorphic function are homologous (6.2).

9.2. TOPOLOGY OF THE BLOW - UP. Let $U=\mathbf{C}^{2}-0$, with coordinates $(x, y)$. Consider the inversion map $t:(x, y) \rightarrow(x /(x \bar{x}+y \bar{y}), y /(x \bar{x}+y \bar{y}))$. Clearly $t^{2}=\mathrm{id}$ and $t$ fixes the lines through the origin. The sphere $S=(x \bar{x}+y \bar{y}=1)$ is the fixed point set of $t$, and $t$ turns $U$ inside out, fixing $S$.

Now complete $\mathbf{C}^{2}$ to $\mathbf{C P}^{2}$ by adding the line $L$ at infinity. Let $U^{+} \subset \mathbf{C P}^{2}$ be the subset given by $L \cup\left\{(x, y) \in \mathbf{C}^{2} \mid x \bar{x}+y \bar{y} \geqslant 1\right\}$. Then $\mathbf{C P}^{2}-U^{+}$is the open ball $(x \bar{x}+y \bar{y}<1)$. The inversion $t$ extends continuously to $L$ (it is mapped to the origin), but it cannot be defined at $0 \in C^{2}$. Let $B$ be the 4-manifold obtained by attaching two copies of $U^{+}$along the boundary $S$. Because orientations do not match along $S$, we reverse the orientation of the second copy. Let $\overline{\mathbf{C P}^{2}}$ denote the 4-manifold $\mathbf{C P}^{2}$ with reversed orientation. Thus $B$ is the connected sum of $\mathbf{C} \mathbf{P}^{2}$ and $\overline{\mathbf{C} \mathbf{P}^{2}}$.

We can define a map $p: B \rightarrow \mathbf{C P}^{2}$ by $p=$ id on the first copy of $U^{+}$and $p=t \circ$ id on the second copy. $p$ is $1: 1$ outside $0 \in \mathbf{C}^{2}$ and the line $\bar{L}$ of the second copy maps to $0 \in \mathbf{C}^{2}$. This $\bar{L}$ corresponds exactly to the lines through the origin; thus $B \approx B_{0} \mathbf{C P}^{2}$.

In $\mathbf{C P}^{2}$ we have $[L][L]=1$, and so in $\overline{\mathbf{C} \mathbf{P}^{2}}$ this becomes $[\bar{L}][\bar{L}]=-1$.

Since locally any two blow-ups look the same, we get the following in general:

If $X$ is a smooth algebraic surface and $x \in X$, then $B_{x} X$ is diffeomorphic to the connected sum of $X$ and $\overline{\mathbf{C P}^{2}}$. If $E \subset B_{x} X$ is the preimage of $x$, then $[E][E]=-1$.

It should be emphasized, however, that $\overline{\mathbf{C P}^{2}}$ cannot be viewed as a complex manifold in any way. Therefore blowing up is not a connected sum among complex manifolds.

9.3. COORDINATES ON THE BLOW - UP. This is easier to work out locally. Let $D$ be the unit ball with coordinates $(x, y)$. Then $B_{0} D$ is defined in $D \times \mathbf{C P}^{1}$ by the equation $t y-x s=0$, where $(t: s)$ are coordinates on $\mathbf{C P}^{1}$. If $s \neq 0$, then in $D \times \mathbf{C}$ we have an equation $x=t y / s$. Thus we can choose $y^{\prime}=y$, $x^{\prime}=t / s=x / y$ as local coordinates. On the $t \neq 0$ chart we can take $x^{\prime \prime}=x$, $y^{\prime \prime}=s / t=y / x$ as local coordinates.

In particular, if $p: B_{0} D \rightarrow D$ is the natural map, then

$$
p^{*}(d x \wedge d y)=y^{\prime} d x^{\prime} \wedge d y^{\prime}=x^{\prime \prime} d x^{\prime \prime} \wedge d y^{\prime \prime} .
$$

Therefore we pick up a simple zero along the curve $E=p^{-1}(0)$. 
9.4. $f^{*}$ ON CURVES. Let $f: X \rightarrow Y$ be a regular birational map between smooth projective surfaces. Let $C \subset Y$ be an algebraic curve. I intend to define a curve $f^{*} C \subset X$ such that $\left[f^{*} C\right]=f^{*}[C]$.

$f^{-1}$ is regular except at finitely many points $\left\{y_{i}\right\}$. Each $f^{-1}\left(y_{i}\right)$ is an algebraic subvariety which is connected by 5.17 and is not a point. Thus these are unions of irreducible curves, called the exceptional curves of $f$. Let these be $\left\{E_{j}\right\}$.

Let $C^{\prime} \subset X$ be the closure of $f^{-1}\left(C-\left\{y_{i}\right\}\right)$. This is called the proper transform of $C$. If $C$ does not pass through any of the $y_{i}$ 's, then clearly we want $f^{*}(C)=C^{\prime}$.

If $C$ is the zero set of a function $g$, then let $C_{\varepsilon}$ be the level set $g=\varepsilon$. We imagine that $C_{\varepsilon}$ moves away from the points $y_{i}$. Thus $f^{*}\left(C_{\varepsilon}\right)=C_{\varepsilon}^{\prime}$, which is a level set of $g \circ f$. Since $\left[C_{\varepsilon}^{\prime}\right]=[\{x \mid g \circ f(x)=0\}]$, the latter should be $f^{*}(C)$. Thus we obtain:

$$
f^{*}(C)=C^{\prime}+\sum m_{j} E_{j}
$$

where $m_{j}$ is the multiplicity of the zeroes of $g \circ f$ along $E_{j}$. Thus $m_{j} \geqslant 0$, and $m_{j}=0$ iff $f\left(E_{j}\right) \notin C$.

9.5. COROLlary. In the above situation if $E$ is an exceptional curve, then $[E] \cdot\left[f^{*} C\right]=0$.

Proof. $f^{*} C$ was defined to be homologous to $C_{\varepsilon}^{\prime}$, which does not intersect any of the exceptional curves; hence $[E]\left[C^{\prime}{ }_{\varepsilon}\right]=0$.

9.6. Corollary. Let $p: B Y \rightarrow Y$ be the blow-up of a point $y \in Y$, and let $E$ be the exceptional curve. Then

$$
c_{1}\left(K_{B Y}\right)=p^{*} c_{1}\left(K_{Y}\right)+[E] .
$$

Therefore $[E] \cdot c_{1}\left(K_{B Y}\right)=-1$.

ProOF. The first statement follows from the computation of 9.3. The last one follows from $[E][E]=-1$, obtained in 9.2.

Now we come to the main result of this chapter, which shows the fundamental importance of blow-ups.

9.7. THEOREM. Let $X$ be a smooth projective surface and $f: X \rightarrow Z$ a rational map of $X$ to some projective variety $Z$. Then there is a finite sequence of blow-ups

$$
g_{n}: X_{n} \rightarrow X_{n-1} \rightarrow \cdots \rightarrow X_{1} \rightarrow X_{0}=X,
$$

such that $f \circ g_{n}$ is everywhere regular.

Proof. We embed $Z$ into some $\mathbf{C P}^{n}$ and let $\left\{H_{t}\right\}$ be the family of hyperplanes. Let $\Gamma \subset X \times Z$ be the closure of the graph of $f$ with projections $p$ and $q$.

Let $\left\{x_{i}\right\}$ be the points where $f$ is not defined. Then $p^{-1}\left(x_{i}\right)$ is a curve in $\Gamma$ by 5.17 , hence $q\left(p^{-1}\left(x_{i}\right)\right)$ is a curve in $\mathbf{C P}^{n}$.

Let $C_{t}=p\left(\Gamma \cap q^{-1}\left(H_{t}\right)\right)$ be the closure of $f^{-1}\left(Z \cap H_{t}\right)$. By 6.5 $\left[H_{t}\right]\left[q\left(p^{-1}\left(x_{i}\right)\right)\right] \neq 0$ and thus $H_{t} \cap q\left(p^{-1}\left(x_{i}\right)\right)$ is not empty. Therefore $C_{t}$ passes through all the $x_{i}$ 's. If $t^{\prime}$ is sufficiently general, then $f(X) \cap H_{t} \cap H_{t^{\prime}}$ 
consists of discrete points only. Thus the same holds for $C_{t} \cap C_{t^{\prime}}$. By 6.4 this implies that $\left[C_{t}\right] \cdot\left[C_{t}\right]=\left[C_{t}\right] \cdot\left[C_{t^{\prime}}\right] \geqslant 0$.

Now let $p_{1}: X_{1} \rightarrow X_{0}$ be the blow-up of one of the $x_{i}$ 's, and let $E$ be the exceptional curve. One can define $C_{t}^{1}$ using the map $f \circ p_{1}$, and from the definitions we see that this is exactly the proper transform of $C_{t}$. By 9.4 we have $p_{1}^{*}\left(C_{t}\right)=C_{t}^{1}+m E$ and $m>0$ since $C_{t}$ passes through the $x_{i}$ 's. Thus

$$
\left[C_{t}^{1}\right]\left[C_{t}^{1}\right]=\left[p_{1}^{*}\left(C_{t}\right)\right]\left[p_{1}^{*}\left(C_{t}\right)\right]-2 m\left[p_{1}^{*}\left(C_{t}\right)\right][E]+m^{2}[E][E] .
$$

On the r.h.s. the first term is the same as $\left[C_{t}\right] \cdot\left[C_{t}\right]$; the second is zero by 9.5 ; and the third is $-m^{2}$ by 9.2 . Thus

$$
\left[C_{t}^{1}\right]\left[C_{t}^{1}\right]=\left[C_{t}\right]\left[C_{t}\right]-m^{2}<\left[C_{t}\right]\left[C_{t}\right]
$$

If $f \circ p_{i}$ is not regular, then similarly one can get $p_{i+1}$ and $C_{t}^{i+1}$. We saw that $\left[C_{t}^{j}\right]\left[C_{t}^{j}\right]$ is a strictly decreasing sequence of integers that are all nonnegative. Therefore the procedure must stop. This proves the theorem.

9.8. COROLlaRY. With the assumptions of 9.7, assume that $f$ is not regular. Then $Z$ contains a rational curve.

PROoF. Let $p_{n}: X_{n} \rightarrow X_{n-1}$ be the last necessary blow-up with exceptional curve $E$. If $f(E)$ were a point, then $f \circ p_{n-1}$ would be regular too. Therefore $f(E)$ is a rational curve in $Z$.

9.9. REMARK. By 8.7 the conclusion still holds if $X$ is an arbitrary-dimensional smooth projective variety. This is another example of the general theme that rational curves cause the trouble.

9.10. Corollary. Let $f: X \rightarrow Z$ be a birational map between smooth projective surfaces. Assume that $[C] \cdot c_{1}\left(K_{Z}\right) \geqslant 0$ for every curve $C \subset Z$. Then $f$ is regular.

Proof. Let again $p_{n}: X_{n} \rightarrow X_{n-1}$ be the last necessary blow-up with exceptional curve $E$. $f(E)$ is not a point; therefore $[E] c_{1}\left(K_{X_{n}}\right) \geqslant$ $[f(E)] c_{1}\left(K_{Z}\right) \geqslant 0$ by 8.14 . On the other hand by $9.6,[E] c_{1}\left(K_{X_{n}}\right)=-1$, a contradiction. Thus $f$ is regular.

9.11. CoRollary. A birational equivalence class of smooth projective surfaces can contain at most one member whose canonical line bundle is nef.

8.7 and 9.7 reduce the problem of studying birational maps to the study of regular birational maps. If $f: X \rightarrow Z$ is a regular birational map, then one can apply 8.7 or 9.7 to $f^{-1}$ and this in turn will give information about $f$ itself. We shall see two results of this kind.

Let $B \subset Z$ be the set where $f^{-1}$ is not regular and let $E=f^{-1}(B) \subset X$.

9.12. Proposition. With the above notation, $E$ is covered by rational curves.

Proof. By 8.7 there is a sequence of blowing-ups $g_{n}: Z_{n} \rightarrow \cdots \rightarrow Z_{0}=Z$ such that $f^{-1} \circ g_{n}$ is regular. By definition each blowing-up introduces an exceptional set which is covered by copies of $\mathbf{C P}^{k-1}$ (8.6(iii)) and hence 
covered by rational curves. The images of these under $f^{-1} \circ g_{n}$ cover $E$, hence the claim.

The following result is more important.

9.13. TheOREM. Let $f: X \rightarrow Z$ be a regular birational map between smooth projective varieties and let $E \subset X$ and $B \subset Z$ be as above. Assume that $f$ is not an isomorphism, i.e., $E \neq \varnothing$. Then there exists a rational curve $D \subset E$ such that [D] $c_{1}\left(K_{X}\right)<0$.

Proof. By $5.19 \operatorname{dim} B \leqslant \operatorname{dim} Z-2$. Pick any $z \in B$ and let $C_{0}$ be a general smooth curve through $z$. If we move $C_{0}$ in a general direction to get a family of curves $\left\{C_{t}\right\}$, then we can assume that $S=\cup C_{t}$ is a smooth surface, $C_{0} \cap B=z$ and $C_{t} \cap B=\varnothing$ for $t$ close to 0 .

Since $f^{-1}: S \rightarrow X$ might not be regular at $z \in C_{0} \subset S$, we use 9.7 to get a sequence of blowing ups $h_{n}: S_{n} \rightarrow S$ such that $p=f^{-1} \circ h_{n}: S_{n} \rightarrow X$ is regular. If $D_{i} \subset S_{n}$ are the $h_{n}$-exceptional curves, then $h_{n}^{-1}\left(C_{0}\right)=C_{0}^{\prime}+\sum m_{i} D_{i}$ $\left(m_{i} \geqslant 0\right)$ and $h_{n}^{-1}\left(C_{t}\right)=C_{t}^{\prime}$ for $t \neq 0$, where $C_{t}^{\prime}$ is the proper transform as in 9.4. In particular, $\left[C_{0}^{\prime}\right]+\sum m_{i}\left[D_{i}\right]=\left[C_{t}^{\prime}\right](t \neq 0)$. Applying $p$ gives

$$
\left[p\left(C_{0}^{\prime}\right)\right]+\sum m_{i}\left[p\left(D_{i}\right)\right]=\left[p\left(C_{t}^{\prime}\right)\right] \quad(t \neq 0) .
$$

Now let us compute intersection numbers with $c_{1}\left(K_{X}\right)$. Since $p\left(C_{0}^{\prime}\right) \not \subset E$ and $p\left(C_{t}^{\prime}\right) \cap E=\varnothing$ if $t \neq 0$, we can use 8.14 to get

$$
\begin{gathered}
{\left[p\left(C_{t}^{\prime}\right)\right] c_{1}\left(K_{X}\right)=\left[f \circ p\left(C_{t}^{\prime}\right)\right] c_{1}\left(K_{Z}\right)=\left[C_{t}\right] c_{1}\left(K_{Z}\right) \quad(t \neq 0) ;} \\
{\left[p\left(C_{0}^{\prime}\right)\right] c_{1}\left(K_{X}\right)>\left[f \circ p\left(C_{0}^{\prime}\right)\right] c_{1}\left(K_{Z}\right)=\left[C_{0}\right] c_{1}\left(K_{Z}\right) .}
\end{gathered}
$$

Since $\left\{C_{t}\right\}$ is a continuous family, this gives

$$
\left[p\left(C_{0}^{\prime}\right)\right] c_{1}\left(K_{X}\right)>\left[p\left(C_{t}^{\prime}\right)\right] c_{1}\left(K_{X}\right) .
$$

Using this for the equality $(*)$, we get that

$$
\sum m_{i}\left[p\left(D_{i}\right)\right] c_{1}\left(K_{X}\right)<0 \text {. }
$$

Therefore, there is at least one $i$ such that $p\left(D_{i}\right)$ is not a point and $\left[p\left(D_{i}\right)\right] c_{1}\left(K_{X}\right)<0$. Since $f \circ p\left(D_{i}\right)=f \circ f^{-1} \circ h_{n}\left(D_{i}\right)=h_{n}\left(D_{i}\right)=z \in B$, we see that $p\left(D_{i}\right) \subset E$. Thus $D=D_{i}$ is the sought after rational curve.

9.14. Summary. We are gradually getting a good picture of a birational equivalence class of varieties. If $f: X^{\prime} \rightarrow X$ is a regular birational map, then it is reasonable to consider $X^{\prime}$ to be "more complicated" than $X$. By blowing up smooth subvarieties we can always make a variety more complicated. 8.7 and 9.7 tell us that this is the way toward arbitrarily complicated varieties. The really interesting task would be to find simpler varieties within the birational equivalence class. 9.13 tells us that if $X^{\prime}$ is more complicated than $X$, then there is a curve $D \subset X^{\prime}$ such that $[D] c_{1}\left(K_{X^{\prime}}\right)<0$; i.e., $K_{X^{\prime}}$ is not nef. At least for surfaces the converse is true; if $K_{X}$ is nef then $X$ is the simplest member of its birational equivalence class (9.10). In higher dimension such an $X$ might not be the unique smallest member, but at least nothing can be smaller than $X$. From this point of view Mori's program is a search for the smallest varieties in a given birational equivalence class. 
10. Mori's program: smooth case. As we saw in the previous chapters, it is easier to handle varieties for which $K_{X}$ is ample or at least nef. The first main part of Mori's program is to pin down a clear geometric reason why $K_{X}$ fails to be nef in any given case.

10.1. Definition. Let $X$ be a smooth projective variety. Recall that $K_{X}$ can be viewed as a linear function on $\overline{N E}(X)$. The intersection

$$
\overline{N E}(X)^{-}=\overline{N E}(X) \cap\left\{z \in H_{2}(X, \mathbf{R}) \mid z c_{1}\left(K_{X}\right)<0\right\}
$$

will be called the negative part of $\overline{N E}(X)$. A closed subcone $V \subset \overline{N E}(X)$ will be called negative if $V-\{0\} \subset \overline{N E}(X)^{-}$.

By a slight abuse of notation, we shall say that an extremal ray is spanned by a curve $C$ if it is spanned by $[C] \in H_{2}(X, \mathbf{R})$.

The following is the first major new result of Mori.

10.2. FirST FundaMental TheOREM. Let $X$ be a smooth projective variety. Then $\overline{N E}(X)^{-}$is locally finitely generated, and each negative extremal ray is spanned by a rational curve $C \subset X$ satisfying $0>[C] \cdot c_{1}\left(K_{X}\right) \geqslant-\operatorname{dim} X-1$.

Proof. If $\overline{N E}(X)^{-}$is empty, there is nothing to prove. Otherwise, there is a curve $D \subset X$ such that $[D] \cdot c_{1}\left(K_{X}\right)<0$. I will outline how to get from $D$ some rational curve $C$. The rest then follows by a technical argument. The first step in finding $C$ is the following.

10.3. Deformation Lemma. Let $g: \bar{D} \rightarrow X$ be the normalization of $D$. Assume that there is an affine smooth curve $P$ and a map $G: \bar{D} \times P \rightarrow X$ such that

(i) $\operatorname{dim} G(\bar{D} \times P)=2$;

(ii) For some $p_{0} \in P, G\left(\cdot, p_{0}\right)=g(\cdot)$;

(iii) For some $d_{0} \in \bar{D}, G\left(d_{0}, \cdot\right)=g\left(d_{0}\right)$;

i.e., we can put $g$ into a nontrivial family of maps $G(\cdot, p): \bar{D} \rightarrow X$ such that the image of $d_{0}$ is fixed.

Then $X$ contains a rational curve.

Proof. We complete $P$ to a projective curve $\bar{P}$. Then $\bar{G}: \bar{D} \times \bar{P} \rightarrow X$ is a rational map. Assume first that it is regular. Then $\bar{G}\left(\left\{d_{0}\right\} \times \bar{P}\right)=$ point, so by the rigidity theorem $(5.23) \bar{G}(\{d\} \times \bar{P})=$ point for every $d \in \bar{D}$. Thus the family is trivial, contradiction.

Therefore $\bar{G}$ cannot be regular, hence by 9.8 we find a rational curve in $X$.

Next we have to concentrate on finding the family of maps $G$. This leads to the general question: if $f: U \rightarrow V$ is a map then how can one change $f$ a little bit?

10.4. Small Deformations. Assume that we are given a family of maps $f_{t}: U \rightarrow V$. Then $\lim \left(f_{t}(u)-f_{0}(u)\right) / t$ gives a tangent vector to $V$ at $f_{0}(u)$. If we let $u \in U$ vary, we get a section of $f_{0}^{*} T_{V}$. Thus if we have a nontrivial family $f_{t}$, then $f_{0}^{*} T_{V}$ certainly has a nontrivial section.

In our original situation we look for sections of $g^{*} T_{X}$. This is a vector bundle of rank $=\operatorname{dim} X$ on a smooth curve. If we imagine it to be a sum of 
line bundles, then Riemann's theorem gives

$$
\begin{aligned}
\operatorname{dim} \Gamma\left(\bar{D}, g^{*} T_{X}\right) & \geqslant \operatorname{deg} g^{*} T_{X}+(1-g(\bar{D})) \operatorname{dim} X \\
& =[D] c_{1}\left(T_{X}\right)+(1-g(\bar{D})) \operatorname{dim} X \\
& =-[D] c_{1}\left(K_{X}\right)+(1-g(\bar{D})) \operatorname{dim} X,
\end{aligned}
$$

and this formula holds in general as well $(g(\bar{D})$ is the genus of $\bar{D})$.

We have one more condition to account for, namely, we do not want $g\left(d_{0}\right)$ to vary. This condition will imply that the section gives the zero vector at $d_{0}$. This imposes rank $g^{*} T_{X}=\operatorname{dim} X$ new conditions; thus if $\Gamma\left(\bar{D}, g^{*} T_{X}\left(-d_{0}\right)\right)$ denotes those sections that vanish at $d_{0}$ we get

$$
\operatorname{dim} \Gamma\left(\bar{D}, g^{*} T_{X}\left(-d_{0}\right)\right) \geqslant-[D] c_{1}\left(K_{X}\right)-g(\bar{D}) \operatorname{dim} X .
$$

An element of $\Gamma\left(\bar{D}, g^{*} T_{X}\left(-d_{0}\right)\right)$ can be considered as a linear approximation to some family of maps $G$. Conversely, one can hope that for any linear approximation there is an actual family with this approximation. This is not quite true, but one can prove that if $-[D] c_{1}\left(K_{X}\right)-g(\bar{D}) \operatorname{dim} X>0$, then there is an actual family $G: \bar{D} \times P \rightarrow X$. I again note that in the $C^{\infty}$ category such a family always exists; the problem is to ensure holomorphy.

10.5. How To MAKE $-[D] c_{1}\left(K_{X}\right)-g(\bar{D}) \operatorname{dim} X$ POSITIVE. The first term is positive by assumption, but we cannot compare it with $g(\bar{D}) \operatorname{dim} X$. We can try to change $\bar{D}$ in some way that will make the first term grow and keep the second fixed.

If $g(\bar{D})=0$, then it is already a rational curve; nothing need be done.

Next assume that $g(\bar{D})=1$; i.e., $\bar{D}$ is elliptic. We take the $n^{2}$-sheeted cover $n: \bar{D} \rightarrow \bar{D}$ given in 3.3(vi) and look at $g \circ n: \bar{D} \rightarrow X$. The image of this is still $D$, but it should be counted with multiplicity $n^{2}$. Thus the relevant formula becomes

$$
\operatorname{dim} \Gamma\left(\bar{D},(g \circ n)^{*} T_{X}\left(-d_{0}\right)\right) \geqslant-n^{2}[D] c_{1}\left(K_{X}\right)-\operatorname{dim} X .
$$

If $n \gg 0$, then the r.h.s. becomes positive and we can use the map $g \circ n$ to find a rational curve.

Now let $g(\bar{D}) \geqslant 2$. Then by 3.5 all self-maps of $\bar{D}$ are $1: 1$. We can still try to look at other covers $p: E \rightarrow \bar{D}$ and study $g \circ p$, but this changes $g(\bar{D})$ to $g(E)$, and one can see that these changes will cancel out. This trick will not work. We desperately need self-maps of $\bar{D}$.

10.6. LAST HOPE, FINITE FIELDS. Assume now for a moment that we suddenly lose $\mathbf{C}$ and find ourselves in a finite field $\mathbf{F}_{p}=\{$ integers $\bmod p\}$. There is no problem doing algebra here and introducing $\mathbf{F}_{p}^{n}, \mathbf{F}_{p} \mathbf{P}^{n}$ and algebraic subvarieties of $\mathbf{F}_{p} \mathbf{P}^{n}$. Polynomials over $\mathbf{F}_{p}$ do not always have roots in $\mathbf{F}_{p}$, and as we saw in the case of $\mathbf{R}$, this leads to trouble. Therefore we introduce $\overline{\mathbf{F}}_{p}=\{$ all roots of polynomials in $\left.\mathbf{F}_{p}[x]\right\}$ and we should rather work with $\overline{\mathbf{F}}_{p} \mathbf{P}^{n}$. One of its remarkable features is the Frobenius map $F\left(x_{0}: \ldots: x_{n}\right)=\left(x_{0}^{p}: \ldots: x_{n}^{p}\right)$. $(x+y)^{p}=x^{p}+y^{p}$ in $\overline{\mathbf{F}}_{p}$ since $(x+y)^{p}=\sum\left(\begin{array}{l}p \\ i\end{array}\right) x^{i} y^{p-i}$ and $p$ divides $\left(\begin{array}{l}p \\ i\end{array}\right)$ except for $i=0, p$. Thus if $f=\sum a_{i_{0}, \ldots, i_{n}} x_{0}^{i_{0}} \cdots x_{n}^{i_{n}}$ is a polynomial in $\overline{\mathbf{F}}_{p}\left[x_{0}, \ldots, x_{n}\right]$, then $f\left(x_{0}, \ldots, x_{n}\right)^{p}=\sum a_{i_{0}, \ldots, i_{n}}^{p}\left(x_{0}^{p}\right)^{i_{0}} \cdots\left(x_{n}^{p}\right)^{i_{n}}$. 
If $a \in \mathbf{F}_{p}$ then $a^{p}=a$. Hence if $f$ is in $\mathbf{F}_{p}\left[x_{0}, \ldots, x_{n}\right]$ (i.e., $a_{i_{0}, \ldots, i_{n}} \in \mathbf{F}_{p}$ ), then $f\left(x_{0}, \ldots, x_{n}\right)^{p}=f\left(x_{0}^{p}, \ldots, x_{n}^{p}\right)$. Therefore if $X \subset \overline{\mathbf{F}}_{p} \mathbf{P}^{n}$ is an algebraic subvariety which is defined by equations in $\mathbf{F}_{p}\left[x_{0}, \ldots, x_{n}\right]$ (instead of $\left.\overline{\mathbf{F}}_{p}\left[x_{0}, \ldots, x_{n}\right]\right)$, then the Frobenius map maps $X$ into itself.

This is all very nice, but what is this good for? Here it becomes crucial that $X$ is not just any complex manifold but a projective variety. We will look at $X$ and $\bar{D} \bmod p$ !

10.7. Reduction mod $p$. Let $X \subset \mathbf{C P}^{n}$ be a projective embedding, and let $X$ be defined by equations $f_{1}=\cdots=f_{k}=0$. Assume for simplicity that the $f_{i}$ all have rational coefficients. Then one can clear denominators and assume that they all have integral coefficients. Now one can reduce everything mod $p$ and look at $X^{p} \subset \overline{\mathbf{F}}_{p} \mathbf{P}^{n}$ defined by $f_{1}=\cdots=f_{k}=0(\bmod p)$.

As we saw in 8.24 , a rational curve $C \subset X$ is given by some polynomial map $\mathbf{C} \rightarrow \mathbf{C P}^{n}, t \rightarrow\left(\sum a_{i}^{0} t^{i}: \ldots: \sum a_{i}^{n} t^{i}\right)$ and the image lies in $X$ iff the polynomials $f_{j}\left(\sum a_{i}^{0} t^{i}, \ldots, \sum a_{i}^{n} t^{i}\right)$ are all identically zero. This leads to a certain system $\Sigma$ of polynomials in the variables $a_{t}^{s}$, with coefficients exactly the coefficients of the $f_{j}$ 's hence integers. Now we use the following principle:

If $\Sigma$ is a system of polynomials with integer coefficients and $\Sigma$ has a solution in $\overline{\mathbf{F}}_{p}$ for every $p$, then $\Sigma$ has a solution in $\mathbf{C}$.

I explain this for linear polynomials. In this case the solvability criterion is the vanishing of certain determinants composed of the coefficients. The condition for solvability in $\overline{\mathbf{F}}_{p}$ is the vanishing of the same determinants $\bmod p$. Clearly an integer is zero iff it is zero $\bmod p$ for every $p$, and this proves the principle in this case. In general one has to find similar solvability criteria for nonlinear systems, a task already completed in the 19th century.

Thus finding rational curves mod $p$ will eventually give us a rational curve on $X$ itself.

One still has the arduous task of developing the whole of algebraic geometry over $\overline{\mathbf{F}}_{p}$. This indeed can be done, and using the Frobenius map $\bar{D}^{p} \rightarrow \bar{D}^{p}$ instead of $n: \bar{D} \rightarrow \bar{D}$ as in 10.5 , one finds a rational curve on $X^{p}$ for every $p$, and thus on $X$.

10.8. REMARKs. (i) This seems indeed a convoluted way of finding rational curves, but this is the only proof so far. Even more remarkably, there is a compact complex threefold $X$ that contains a curve $D$ such that $[D] c_{1}\left(K_{X}\right)<0$ but $X$ contains no rational curves.

(ii) There are two points to be tidied up in 10.7. First of all, what if the $f_{i}$ do not have rational coefficients? Let $\left\{b_{j}\right\}$ be all the coefficients of the $f_{i}$ 's and consider the ring $\mathbf{Z}\left[b_{j}\right] \subset \mathbf{C}$. If $P \subset \mathbf{Z}\left[b_{j}\right]$ is any maximal ideal, then $\mathbf{Z}\left[b_{j}\right] / P$ is a finite field. Thus we can reduce $X$ modulo $P$, and work as earlier.

The other point is more delicate. If we want to find a polynomial map $\mathbf{C} \rightarrow \mathbf{C P}^{n}$ given by degree $m$ polynomials, then the system of equations depends on the choice of $m$. Thus in order to succeed we not only have to find a polynomial map $\overline{\mathbf{F}}_{p} \rightarrow X^{p}$ for every $p$, but we have to find maps that are given by polynomials of degree at most $m$. This somewhat technical step relies on the following observation:

If $C \subset X$ is a rational curve such that $[C] c_{1}\left(K_{X}\right)<-n-1$, then the above considerations show that one can deform $C$ keeping two points fixed. As in 
10.3 we will find a new rational curve $C^{\prime}$, and one can prove that $\left[C^{\prime}\right] c_{1}\left(K_{X}\right)$ $>[C] c_{1}\left(K_{X}\right)$. This also explains the last inequality in the statement of the theorem.

(iii) We have some control over the location of the rational curve. A quick look at the proofs of $10.3,9.8$, and 9.7 shows that the rational curve will pass through $d_{0}$. This implies the following

10.9. Theorem. Let $X$ be a smooth projective variety. Assume that $-K_{X}$ is ample. Then $X$ is covered by rational curves.

Proof. Given $d_{0} \in X$, pick a curve $D$ through $d_{0}$. Then $[D] c_{1}\left(K_{X}\right)<0$. Hence by 10.8(iii) there is a rational curve passing through $d_{0}$.

The next major result is the complete description of the contractions associated to negative extremal rays for $\operatorname{dim} X \leqslant 3$. We start with the twodimensional case.

10.10. Second Fundamental Theorem $(\operatorname{dim} X=2)$. Let $X$ be $a$ smooth projective surface and $C \subset X$ a rational curve which generates an extremal ray. By $10.20>[C] c_{1}\left(K_{X}\right) \geqslant-3$. Then this ray can be contracted (6.12), and we are in one of the following situations.

(i) $[C] c_{1}\left(K_{X}\right)=-3$. Then $X=\mathbf{C P}^{2}, C \subset X$ is a line, and $f: X \rightarrow$ point is the contraction map.

(ii) $[C] c_{1}\left(K_{X}\right)=-2$. Then the contraction map is $f: X \rightarrow E$, where $E$ is a smooth projective curve and all the fibers are isomorphic to $\mathbf{C P}^{1} ; C$ is any one of them.

(iii) $[C] c_{1}\left(K_{X}\right)=-1$. Then $X=B_{y} Y$ for some smooth surface $Y, f: B_{y} Y \rightarrow Y$ is the contraction, and $C$ is the exceptional curve of $f$.

10.11. Corollary. Let $X$ be a smooth projective surface. Then there is a smooth projective surface $Y$ such that $X$ can be obtained from $Y$ by repeatedly blowing up points and $Y$ satisfies exactly one of the following conditions:

(i) $Y \cong \mathbf{C P}^{2}$;

(ii) $Y$ has a map onto a curve $E$ such that all fibers are isomorphic to $\mathbf{C P}^{1}$;

(iii) $K_{Y}$ is nef.

Proof. If $K_{X}$ is nef, then take $Y=X$. If not, then contract an extremal ray. If we are in the situation (i) or (ii) of 10.10, then again take $Y=X$. If not, then we are in case (iii) and $X=B X_{1}$ for a surface $X_{1}$. Now continue the procedure. We will stop after at most $\operatorname{dim} \mathrm{H}_{2}(X, \mathbf{R})$ steps.

The birational equivalences of types (i) and (ii) can be analyzed very easily. One gets that $\mathbf{C} \mathbf{P}^{2}$ is birational to $\mathbf{C} \mathbf{P}^{1} \times \mathbf{C} \mathbf{P}^{1}$, that in case (ii) $Y$ is birational to $E \times \mathbf{C P}^{1}$, and that $E$ is unique. Now we can put together 9.12 and 10.11 as follows:

10.12. COROllaRY. Let $X$ be a smooth projective surface. Then either

(i) $X$ is birationally equivalent to a unique $E \times \mathbf{C P}^{1}$ and so $X$ is covered by rational curves and $P_{m}(X)=0$ for every $m \geqslant 1$; or

(ii) The birational equivalence class of $X$ contains a unique $\bar{X}$ such that $K_{\bar{X}}$ is nef. $X$ can be obtained from $\bar{X}$ by repeatedly blowing up points. 
This is the dichotomy that was alluded to in 8.2.

10.13. COROllary. Let $X$ and $Y$ be birationally equivalent smooth projective surfaces. Consider the following statements.

(i) $X$ and $Y$ are homeomorphic.

(ii) $X$ and $Y$ are diffeomorphic.

(iii) $\operatorname{dim} H_{2}(X, \mathbf{R})=\operatorname{dim} H_{2}(Y, \mathbf{R})$.

Then (i) $\Leftrightarrow$ (ii) $\Rightarrow$ (iii) and (iii) $\Rightarrow$ (ii) except when $\operatorname{dim} H_{2}(X, \mathbf{R})=2$ and both $X$ and $Y$ are $S^{2}$ bundles over a topological surface, in which case there are two diffeomorphism classes.

Proof. If we are in case 10.12(ii), then by 9.2 we know that $X$ is diffeomorphic to the connected sum of $\bar{X}$ and a few copies of $\overline{\mathbf{C P}}{ }^{2}$, and the same holds for $Y$. This implies the result.

The case 10.12(i) requires a little bit more work, but it is mostly easy topology using 10.11 . The reader might put together the proof himself.

Theorem 10.10 also gives a complete description of regular birational maps in dimension two.

10.14. Theorem. Let $g: X \rightarrow Z$ be a regular birational map between smooth projective surfaces. Then $g$ is the composite of blowing-downs.

Proof. We saw in 9.13 that there is a curve $C \subset X$ such that $g(C)=$ point and $[C] c_{1}\left(K_{X}\right)<0$. One can easily see that this must be one of the extremal curves found in 10.2. Now apply 10.10. In cases (i) or (ii), the curve $C$ moves in a family $\left\{C_{t}\right\}$ and so $g\left(C_{t}\right) \subset Z$ would be homologous to $g(C)=$ point, a contradiction. Thus $X=B_{y} Y$ and clearly $g$ factors as $X \rightarrow Y \rightarrow Z$. Now repeat this with $Y \rightarrow Z$ to get a factorization of $g$ into blowing-downs.

10.15. REMARK. I should emphasize that 10.11-10.14 are not new. They were known to geometers around the turn of the century. Mori's program, however, provided a completely new and unified approach. The three possibilities in 10.11 used to be considered as three completely separate theorems. It is only now that we can view them as three cases of a single result.

Now consider the three-dimensional case. These results of Mori were not even conjectured before.

10.16. Second Fundamental Theorem $(\operatorname{dim} X=3)$. Let $X$ be a smooth projective threefold and $C \subset X$ a rational curve which generates an extremal ray. Then this ray can be contracted, and we are in one of the following situations, where $f: X \rightarrow Z$ denotes the contraction map.

(i) $Z=$ pt. Such examples are $X=$ hypersurface in $\mathbf{C P}^{4}$ of degree $\leqslant 4$. There are $a$ few other cases, all of them classified by Iskouskih-Fano. All are covered by rational curves.

(ii) $Z=$ smooth curve. Such examples are $\mathbf{C P}^{2} \times Z$ and a few others, all understood, again covered by rational curves.

(iii) $Z=$ smooth surface, all the fibers are conics in $\mathbf{C P}^{2}$, again covered by rational curves.

(iv) $f$ is the inverse of a blowing-up of $Z$ of one of the following types:

(a) $Z$ smooth, $X=B_{E} Z$ for a smooth curve $E \subset Z$;

(b) $Z$ smooth, $X=B_{z} Z$ for some $z \in Z$; 
(c) Z has one singular point $z$ of the form

$$
\left(x^{2}+y^{2}+z^{2}+u^{2}=0\right) \subset \mathbf{C}^{4}
$$

and $X=B_{z} Z$;

(d) $Z$ has one singular point $z$ of the form

$$
\left(x^{2}+y^{2}+z^{2}+u^{3}=0\right) \subset \mathbf{C}^{4}
$$

and $X=B_{z} Z$

(e) $Z$ has one singular point $z$ of the form

$$
\mathbf{C}^{3} /(x, y, z) \sim(-x,-y,-z)
$$

and $X=B_{z} Z$.

10.17. REMARK. The cases do not correspond to the values of $[C] c_{1}\left(K_{X}\right)$ as it happened in 10.10 .

10.18. Summary. This accomplishes the first main goal of Mori's program. If $K_{X}$ is not nef, then there is a clear and simple geometric reason for it. 'This reason can be a global one or a local one. In the global case the rational curves generating the extremal ray cover $X$, and one obtains very good structure theorems. These are the cases 10.10(i) and (ii) and 10.16(i)-(iii). In the local case the rational curves fill out a proper subvariety only, which we can contract. For surfaces this gives again a smooth surface which is simpler than the one we started with. The procedure can be repeated and gives a very nice structure theorem 10.11 .

The threefold case is more complicated since a contraction can lead to singularities, and it is not clear how to continue. The way ahead is to understand that we have to put up with certain kinds of simple singularities. Once the proper class of singularities is found, one should develop an analog of the preceding results in this more general setting. This will be done in the next chapter.

11. Mori's program: singular case. As we saw at the end of the previous chapter, we are led to formulate some analog of Mori's program for singular varieties even if we are interested mainly in the smooth case. For arbitrary singularities, however, the set-up does not even make sense. We have to choose our class of singularities very carefully. There are two main conditions to be imposed.

11.1. First CONDITION. By definition, the main point of the program is to investigate varieties $X$ where $K_{X}$ is not nef. We cannot hope to do this unless $K_{X}$ exists and we can define a number $[C] c_{1}\left(K_{X}\right)$ for curves $C \subset X$.

Let $\Sigma \subset X$ be the set of singularities. Then $X-\Sigma$ is smooth; hence $K_{X-\Sigma}$ can be defined in the usual manner. Now it is straightforward to try to define $K_{X}$ as a line bundle on $X$ such that $K_{X} \mid X-\Sigma \cong K_{X-\Sigma}$. Such a $K_{X}$ would then be unique provided $X$ is normal, which we shall assume always. Once we have uniqueness the existence of $K_{X}$ becomes a local problem around $s \in \Sigma$. This can be checked using the following. 
11.2. Lemma. Let $X$ be a normal algebraic variety and let $S \subset X$ be a subvariety such that $\operatorname{dim} S \leqslant \operatorname{dim} X-2$. Let $M$ be a line bundle on $X-S$. The following are equivalent:

(i) There is a unique line bundle $L$ on $X$ such that $L \mid X-S \cong M$.

(ii) Every $s \in S$ has a neighborhood $U \subset X$ such that $M \mid U-S \cap U$ has a nowhere zero section.

Proof. (i) $\Rightarrow$ (ii). $L$ is locally trivial in a neighborhood of any $s$; in particular, it has a nowhere zero section in that neighborhood.

(ii) $\Rightarrow$ (i). First, I claim that $L$ is unique. Indeed, if $L_{1}$ and $L_{2}$ are two extensions, then $N=L_{1} \otimes L_{2}^{-1}$ is a line bundle on $X$ such that $N \mid X-S \cong$ $M \otimes M^{-1} \cong \mathcal{O}_{X-S}$. The constant section of $\mathcal{O}_{X-S}$ gives a section of $N$ over $X-S$ which extends to a section $s$ of $N$ over $X$ by 7.5. By the same argument $s^{-1}$ is a section of $N^{-1}$ over $X$; therefore $s$ is nowhere zero. Hence by 7.8(ii), $N \cong \mathcal{O}_{X}$ and $L_{1} \cong L_{2}$.

Once we know that $L$ is unique, it is sufficient to construct it locally. $M \mid U-S \cap U \cong \mathcal{O}_{U-S \cap U}$ by 7.8(ii) and therefore $\mathcal{O}_{U}$ gives an extension. This completes the proof.

11.3. Proposition. Let $X$ be a hypersurface given by the equation $f\left(x_{1}, \ldots, x_{k}\right)=0$. Let $\Sigma=\operatorname{Sing} X$ and assume that $\operatorname{dim} \Sigma \leqslant \operatorname{dim} X-2$. Then $K_{X}$ exists.

Proof. By 5.8, $X$ is normal. We shall check condition (ii) of 11.2. Let $U_{i} \subset X$ be the subset where $\partial f / \partial x_{i} \neq 0$. As we saw in $2.25, X-\Sigma=\cup U_{i}$. We shall define a nowhere zero section of $K_{X-\Sigma}$ as follows. On $U_{i}$ we pick

$$
t_{i}=(-1)^{i}\left(\frac{\partial f}{\partial x_{i}}\right)^{-1} d x_{1} \wedge \cdots \wedge d x_{i-1} \wedge d x_{i+1} \wedge \cdots \wedge d x_{k} .
$$

This is a nowhere zero section of $K_{U_{i}}$. From $f=0$ we get $\Sigma \partial f / \partial x_{i} \cdot d x_{i}=0$ on $X$, and from this we obtain that $t_{i}$ and $t_{j}$ agree on $U_{i} \cap U_{j}$. Thus they define a nowhere zero section of $K_{X-\Sigma}$; hence by 11.2 we conclude that $K_{X}$ exists.

This indeed takes care of the cases 10.16(iv)(c) and (d), but fails to cover the case (e). In fact, in that case $K_{X}$ does not exist. For that case we need the following:

11.4. Proposition. Let $U$ be an algebraic variety such that $K_{U}$ exists. Assume that a finite group $G$ of order $g$ acts on $U$ and $V=U / G$. Let $S^{\prime} \subset U$ be the set of points fixed by at least one nonidentity element of $G$. Assume that $\operatorname{dim} S^{\prime} \leqslant$ $\operatorname{dim} U-2$. Then there is a line bundle $L$ on $V$ such that

$$
L \mid(V-\operatorname{Sing} V) \cong K_{V-\operatorname{Sing} V}^{\otimes},
$$

i.e., $K_{V}$ might not exist, but $K_{V}^{\otimes g}$ does.

Proof. Let $p: U \rightarrow V$ be the quotient map and let $S=p\left(S^{\prime}\right)$. Then $p: U-S^{\prime} \rightarrow V-S$ is a local analytic isomorphism and each $v \in V-S$ has exactly $g$ preimages. The line bundle $K_{V-S}$ exists, and if $p(u)=v$ then the natural map $p^{*}: K_{v} \rightarrow K_{u}$ is an isomorphism between the line of $K_{V}$ at $v$ and the line of $K_{U}$ at $u$. 
If $L$ exists, then it is unique. Therefore it is sufficient to prove its existence locally. Thus one can assume that $K_{U}$ has a nowhere zero section $f: U \rightarrow K_{U}$. If $v \in V-S$ and $p^{-1}(v)=\left\{u_{1}, \ldots, u_{g}\right\}$, then $f\left(u_{i}\right) \in K_{u_{t}}$ can be identified with an element of $K_{v}$ via $p^{*}$ as above. Therefore $f$ induces a $g$-valued nowhere zero section of $K_{V-S}$, and this in turn yields a single-valued nowhere zero section of $K_{V-S}^{\otimes g}$. Therefore by $11.2 K_{V-S}^{\otimes g}$ extends to a line bundle $L$ over $V$.

Now let $X$ be a normal projective variety with singular set $S$. Let $L$ be a line bundle on $X$ and $M$ a line bundle on $X-S$. Assume that $L \mid(X-S) \cong$ $M^{\otimes k}$ for some $k>0$. If $C \subset X$ is a curve, then one can formally define

$$
[C] \cdot c_{1}(M)=\frac{1}{k}[C] c_{1}(L) \text {. }
$$

This is a rational number. In particular, if every $x \in X$ has a neighborhood $V$ such that $V \cong U / G$ as in 11.4 , then one can define $[C] c_{1}\left(K_{X}\right)$. This covers the remaining case 10.16 (iv)(e).

11.5. SECOND CONDITION. One of the main reasons for our interest in plurigenera and $m$-canonical maps is their independence of the birational model. We would like to keep this nice property for the singular varieties as well. The following observation leads to the definition of plurigenera in general.

Let $L$ be a line bundle on $X$, and let $\Sigma \subset X$ be the singular set. If $X$ is normal (in particular, $\operatorname{dim} \Sigma \leqslant \operatorname{dim} X-2$ ), then by 7.5 ,

$$
\Gamma(X, L) \cong \Gamma(X-\Sigma, L \mid X-\Sigma) \text {. }
$$

Therefore for an arbitrary variety $X$ we can define plurigenera by

$$
P_{m}(X)=\operatorname{dim} \Gamma\left(X-\operatorname{Sing} X, K_{X-\operatorname{Sing} X}^{\otimes m}\right) .
$$

11.6. Definition. Let $X$ be a projective variety, $x \in X$. We say that $x \in X$ does not affect the plurigenera if the following holds:

For any birational regular map $f: Y \rightarrow X$ with $Y$ smooth and any small neighborhood $U$ of $s$ the natural map

$$
\Gamma\left(f^{-1}(U), K_{f^{-1}(U)}^{\otimes m}\right) \rightarrow \Gamma\left(f^{-1}(U-B), K_{f^{-1}(U-B)}^{\otimes m}\right) \cong \Gamma\left(U-B, K_{U-B}^{\otimes m}\right)
$$

is an isomorphism for every $m \geqslant 1$. Here $B \subset X$ is the set where $f^{-1}$ is not defined.

$U-B \cong f^{-1}(U-B)$; thus any section $s$ of $K_{U-B}^{\otimes m}$ gives a section $\bar{s}$ of $K_{f^{-1}(U-B)}^{\otimes m} . \bar{s}$ will extend to a possibly meromorphic section $f^{*} s$ of $K_{f^{-1}(U)}^{\otimes m}$. Thus the above definition is equivalent to demanding that $f *_{s}$ be a regular section of $K_{f^{-1}(U)}^{\otimes} m$.

Now we can give the following basic definition, due to Reid.

11.7. Definition. A singularity is called canonical if $K^{\otimes m}$ exists for some $m \geqslant 1$ and the singularity does not affect the plurigenera.

At first this seems a very complicated and not too geometric definition to work with, but canonical singularities turn out to form a very nice class. In dimension two this very class of singularities was studied and classified by DuVal in the thirties. (Then they were promptly forgotten and reinvented in the sixties by people working with singularities of $C^{\infty}$ functions.) 
11.8. THEOREM. The canonical surface singularities are exactly the following:

(i) $x^{2}+y^{2}+z^{k}$ for $k \geqslant 1$.

(ii) $x^{2}+z y^{2}+z^{k}$ for $k \geqslant 3$.

(iii) $x^{2}+y^{3}+z^{4}$ or $x^{2}+z y^{3}+z^{3}$ or $x^{2}+y^{3}+z^{5}$.

Although for the purpose of birational geometry canonical singularities turn out to be the right class to consider, they are too big for Mori's program. Indeed in the surface case no singularities were necessary. We can impose further conditions on the singularities to arrive at the class we really need.

11.9. Definition. (i) Notation as in 11.6. We say that $x \in X$ is terminal if $K_{X}^{\otimes m}$ exists for some $m>0$ and the following strengthening of 11.6 holds: for any $s \in \Gamma\left(U-B, K_{U-B}^{\otimes m}\right), f^{*}$ is a section of $K_{f^{-1}(U)}^{\otimes m}$ and it vanishes along every $(\operatorname{dim} X-1)$-dimensional component of $f^{-1}(B)$. (By 2.27 we cannot hope for vanishing along lower-dimensional components.) 8.12 shows that a smooth point is terminal.

(ii) A variety $X$ is said to be $\mathbf{Q}$-factorial if for every irreducible subvariety $H \subset X$ such that $\operatorname{dim} H=\operatorname{dim} X-1$ there is a line bundle $L$ and a section $s: X \rightarrow L$ such that $H=(s=0)$ (as sets, $s$ might have a multiple zero along $H$ ). This condition always holds if $X$ is smooth (7.6(iii)). See 4.4 for a case when it is not satisfied. (The name comes from the fact that this condition is closely related to unique factorization in certain rings of regular functions on open sets of $X$.)

This is a very convenient technical condition that will be satisfied in the cases that interest us.

A two-dimensional surface with terminal singularities is necessarily smooth. In dimension three we have a complete structure theory due to Reid, Mori, Danilov, Morrison and Stevens:

11.10. THEOREM. Three-dimensional terminal singularities are the following

(i) smooth points;

(ii) isolated singularities given by an equation of the form $f(x, y, z)+$ $\operatorname{tg}(x, y, z, t)$, where $f$ is one of those listed in 11.8 ;

(iii) a few of the cyclic quotients of the above (there is a complete list).

Now we are ready to formulate the analog of the First Fundamental Theorem, and a bit of the Second one, for singular varieties. Mori's original ideas about finding rational curves do not seem to work in the general case. Several new ideas had to be developed. The first result was obtained by Kawamata. Further contributions by Benveniste, Kawamata, Kollár, Reid, and Shokurov led finally to the following theorem:

11.11. First Fundamental TheOREM. Let $X$ be a projective variety with canonical singularities. Then $\overline{N E}(X)^{-}$is locally finitely generated, and each negative extremal subcone can be contracted.

11.12. REMARK. It is not yet proved that every extremal ray is spanned by a rational curve although this is true for $\operatorname{dim} X=3$.

The second fundamental theorem of the smooth case gave a complete description of the contraction maps. While in general one cannot expect to list all possibilities, it is reasonable to hope to get more detailed information than 
the mere existence of the contraction. Our current knowledge does not deserve to be called a theorem.

11.13. Second Fundamental Proposition-Definition. Let $X$ be $a \mathbf{Q -}$ factorial projective variety with canonical (resp. terminal) singularities and let $f: X \rightarrow Y$ be the contraction of a negative extremal ray. Then we are in one of the following cases:

(i) Fano contraction: $\operatorname{dim} Y<\operatorname{dim} X$. Then $X$ is covered by rational curves.

(ii) Divisorial contraction: $f$ is birational and there is a subvariety $E \subset X$ such that $\operatorname{dim} E=\operatorname{dim} X-1$ and $\operatorname{dim} f(E)<\operatorname{dim} E$. In this case $Y$ is again Q-factorial with canonical (resp. terminal) singularities.

(iii) Small contraction: $f$ is birational and there is $E \subset X$ such that $\operatorname{dim} E \leqslant$ $\operatorname{dim} X-2$ and $f: X-E \rightarrow Y-f(E)$ is an isomorphism. In this case $K_{Y}^{\otimes m}$ never exists.

Proof. Assume for simplicity that $X$ is smooth. Let $[C]$ span the contracted extremal ray.

First consider the case when $\operatorname{dim} Y<\operatorname{dim} X$. By Sard's theorem then $F=$ $f^{-1}(y) \subset X$ is smooth for general $y \in Y$. Clearly $N_{F \mid X} \cong F \times \mathbf{C}^{\operatorname{dim} Y}$ and hence by 7.6(ii) $K_{F} \cong K_{X} \mid F$. If $D \subset F$ is any curve then $f(D)=$ point. Hence $[D]=\lambda[C]$. In particular, $[D] c_{1}\left(K_{F}\right)=[D] c_{1}\left(K_{X}\right)=\lambda[C] c_{1}\left(K_{X}\right)<0$; thus by $10.9, F$ is covered by rational curves and so is $X$.

Otherwise $\operatorname{dim} Y=\operatorname{dim} X$. Since $f$ has connected fibers it is generically $1: 1$ so it is birational. Let $B \subset Y$ be the set where $f^{-1}$ is not defined and let $E=f^{-1}(B)$. Assume first that there is an irreducible $H \subset E$ such that $\operatorname{dim} H$ $=\operatorname{dim} X-1$. I claim that $[C][H]<0$.

To see this let us look at a family of curves $\left\{D_{t}\right\} \subset Y$ such that $D_{t} \cap B=\varnothing$ for $t \neq 0$ and $D_{0} \cap B=y \in Y$. Then $D_{t}^{\prime}=f^{-1}\left(D_{t}\right)$ is a family of curves for $t \neq 0$ and its limit for $t=0$ is $D_{0}^{\prime}+F$, where $D_{0}^{\prime}$ is the proper transform of $D_{0}$ and $f(F)=y$. We can arrange that $D_{0}^{\prime} \cap H \neq \varnothing$. Since $H$ and $D_{t}^{\prime}$ are disjoint, $\left[D_{t}^{\prime}\right][H]=0$. So $[F][H]=-\left[D_{0}^{\prime}\right][H]<0$. Since $f(F)=$ point, $[F]=$ $\lambda[C]$ and so $[C][H]<0$ as claimed.

Now if $E-H$ is not empty, then we can pick a curve $G \subset E$ such that $f(G)=$ point and $G \not \subset H$. This would give $[G][H] \geqslant 0$, which is a contradiction since $[G]=\mu[C]$ and $\mu>0$. Thus $E=H$.

We will check that $Y$ has canonical singularities. First we prove that $K_{Y}^{\otimes m}$ exists for some $m>0$. If it exists then $f^{*}\left(K_{Y}^{\otimes m}\right)$ is a line bundle which agrees with $K_{X}^{\otimes m}$ on $X-E$ and is trivial along each fiber of $f$. Now consider the line bundle $\mathcal{O}(H)$ given in 7.6(iv). (This is the only point where Q-factoriality is used in general.) Since $\mathcal{O}(H) \mid X-H \cong \mathcal{O}_{X-H}$, any line bundle of the form $L_{m, k}=K_{X}^{\otimes m} \otimes \mathcal{O}(H)^{\otimes k}$ is isomorphic to $f^{*}\left(K_{Y}^{\otimes m}\right)$ on $X-H$. If we pick $k=[C] c_{1}\left(K_{X}\right)$ and $m=-[C] c_{1}(\mathcal{O}(H))=-[C][H]$, then $[C] c_{1}\left(L_{m, k}\right)=0$. Thus $c_{1}\left(L_{m, k}\right)$ is trivial along each fiber of $f$. This implies that $K_{Y}^{\otimes m}$ exists as a topological line bundle. Its existence as a holomorphic line bundle is a little more delicate question and we will not discuss it. A similar argument shows that $Y$ is $\mathbf{Q}$-factorial.

In order to check that the singularities of $Y$ do not affect the plurigenera, let $U$ be a small neighborhood of some $b \in B \subset Y$ and let $s \in \Gamma\left(U-B, K^{\otimes m}\right)$. 
$f^{*} s$ is a meromorphic section of $\Gamma\left(f^{-1}(U), K^{\otimes m}\right)$. Assume that it has a pole of order $t \geqslant 0$ along $H$. Then $c_{1}\left(K_{f^{-1}(U)}^{\otimes m}\right)=[Z]-t[H]$, where $Z$ is the set of zeroes of $f^{*} s$. By assumption $H \not \subset Z$. Pick a curve $D \subset H$ such that $f(D)=$ point and $D \not \subset Z$. Then $[D]=\lambda[C]$. Hence

$$
\lambda m[C] c_{1}\left(K_{f^{-1}(U)}\right)=[D] c_{1}\left(K_{f^{-1}(U)}^{\otimes m}\right)=[D][Z]-t[D][H] \geqslant 0,
$$

a contradiction. Thus $f^{*} s$ will in fact vanish along $H$.

Now let $h: X^{\prime} \rightarrow X$ be a resolution of singularities. $f^{*} s$ is a regular section of $K_{f^{-1}(U)}^{\otimes m}$ and thus $h^{*} f^{*} s$ is a regular section of $K_{h^{-1}}^{\left.\otimes f^{-1}(U)\right)}$ since $X$ has canonical singularities. This proves that $b \in X$ does not affect the plurigenera. One can in fact see in this way that $b \in B \subset X$ is terminal.

The remaining case is when $f$ is birational and $\operatorname{dim} E \leqslant \operatorname{dim} X-2$. In this case $f^{*} s$ extends automatically across $E$ by 7.5 ; hence the singularities of $Y$ do not affect the plurigenera. Unfortunately, $K_{Y}^{\otimes m}$ does not exist. If it did, then $f^{*}\left(K_{Y}^{\otimes m}\right)$ and $K_{X}^{\otimes m}$ would give two extensions of $K_{X-E}^{\otimes m}$ to $X$. By 11.2 such an extension is unique, and hence $f^{*} K_{Y}^{\otimes m}=K_{X}^{\otimes m}$. This is however not the case, since $[C] c_{1}\left(K_{X}^{\otimes m}\right)<0$.

11.14. REMARKS. (i) For surfaces and smooth threefolds only the first two cases occur.

(ii) The easiest example for (iii) is the following: take $\mathbf{C P}^{2}$ and on it the rank two vector bundle $V=\mathcal{O}(-1)+\mathcal{O}(-1)$. Complete $V$ somehow to a smooth projective variety $V \subset X$. The line $l \subset \mathbf{C P}^{2} \subset V \subset X$ generates a negative extremal ray. The contraction map maps $\mathbf{C} \mathbf{P}^{2}$ to a point and is an isomorphism outside it. In this example $\operatorname{dim} X=4$. For smooth fourfolds this is the only known example of small contraction.

(iii) There are many examples of small contractions for singular threefolds, but they are all hard to describe (see 12.5).

11.15. Summary. We achieved the goals set forth at the end of the previous chapter. Unfortunately, the results are not sufficient to complete our progam. Case 11.13(i) is a satisfactory end; it gives good structural information about $X$. If the case 11.13(ii) holds, then $Y$ can be considered as simpler than $X$ and the program can continue. For 11.13(iii), however, the resulting singularity is very bad, and a different idea is needed to analyze the situation. This is the problem that we investigate next.

12. Flip and flop. This section gives some examples and a lot of handwaving about small contractions in dimension three. As I mentioned, all examples are fairly complicated; therefore I will concentrate on a related simpler one.

12.1. EXAMPLE. Let $Q \subset \mathbf{C P}^{3}$ be the quadric given by the equation $x y-u v$ $=0$. As we saw in 4.6, this is isomorphic to $\mathbf{C} \mathbf{P}^{1} \times \mathbf{C P} \mathbf{P}^{1}$, and the two families of lines are given by $(x=\lambda u, v=\lambda y$ ) (for $\lambda=\infty$ this is $u=y=0$ ) and $(x=\lambda v, u=\lambda y)$ (for $\lambda=\infty$ this is $v=y=0$ ).

Now let $C^{0}$ be the cone over $Q$ given by the equation $(x y-u v=0) \subset \mathbf{C}^{4}$. This has one singular point at the origin. We also introduce $C \subset \mathbf{C P}^{4}$, the closure of $C^{0}$; then $Q$ can be considered as $C \cap H$ where $H$ is the hyperplane at infinity. $C$ has still only one singular point at the origin 0 of $\mathbf{C}^{4}$ and we set out to resolve it. We shall do it in three ways. 
(i) First resolution. We blow up the origin 0 in $\mathbf{C P}^{4}$ and look at the closure $C_{12}$ of $C-0$ in $B_{0} \mathbf{C P}{ }^{4}$. Above the origin we get a copy $\bar{E}$ of $\mathbf{C P}^{3}$ in $B_{0} \mathbf{C P}^{4} . C$ is the union of lines connecting thr origin and $Q \subset H$. Thus $E=\bar{E} \cap C_{12}$ is isomorphic to $Q$. Now it is easy to see that $C_{12}$ is smooth.

(ii) In order to get the two other resolutions, we concentrate on the two families of planes on $C$ obtained from the two families of lines. Let

$$
P_{\lambda}^{1}=(x=\lambda u, v=\lambda y), \quad P_{\lambda}^{2}=(x=\lambda v, u=\lambda y) .
$$

Let $\bar{P}_{\lambda}^{i}$ be the closure of $P_{\lambda}^{i}-0$ in $C_{12}$. Since we blew up the origin, clearly $\bar{P}_{\lambda}^{i} \cong B_{0} P_{\lambda}^{i}$, and the exceptional curves on $\bar{P}_{\lambda}^{i}$ give the two families of lines on $E \cong \mathbf{C P}^{1} \times \mathbf{C P}^{1}$. If we fix $i$ and let $\lambda$ vary, then $C_{12}$ is covered by the family $\left\{\bar{P}_{\lambda}^{i}\right\}$ and $\bar{P}_{\lambda}^{i}$ and $\bar{P}_{\mu}^{i}$ are disjoint if $\lambda \neq \mu$. Since $E \cap \bar{P}_{\lambda}^{i}$ can be contracted in each $\bar{P}_{\lambda}^{i}$ to get $P_{\lambda}^{i}$, it is reasonable to assume (and it is true) that this contraction can be done in a family to get a $q_{i}: C_{12} \rightarrow C_{i}$, where $C_{i}$ is the disjoint union of the planes $P_{\lambda}^{i}$. We can picture the situation as shown in Figure 3.

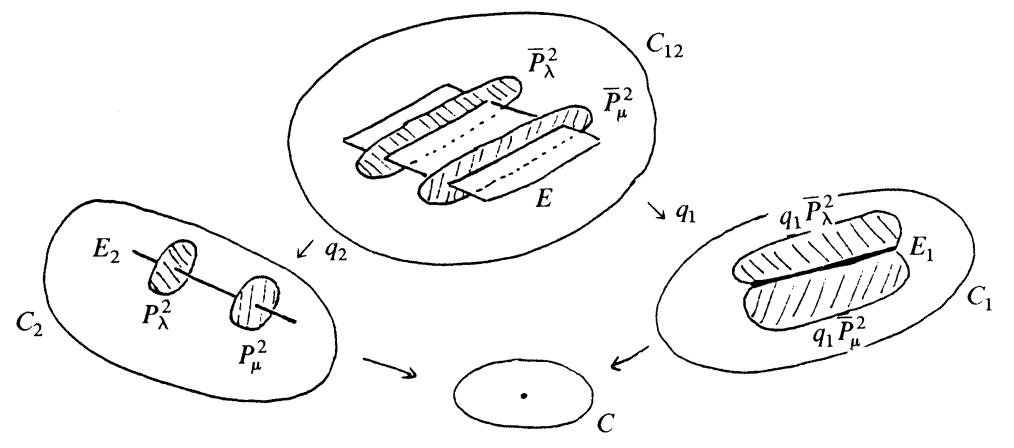

FIGURE 3

From the construction it is clear that $P_{\lambda}^{i} \subset C_{i}$ intersects $E_{i}$ in one point; hence $\left[E_{i}\right]\left[P_{\lambda}^{i}\right]=1$. Let $j=3-i$. $\bar{P}_{\lambda}^{j}$ intersects $E$ in a line that maps onto $E_{i}$. Therefore $q_{i}\left(\bar{P}_{\lambda}^{j}\right)$ contains $E_{i}$ and thus the intersection number $\left[E_{i}\right]\left[q_{i} \bar{P}_{\lambda}^{j}\right]$ is not geometrically clear. To get this number, we note that since $\bar{P}_{\lambda}^{j}$ and $\bar{P}_{\mu}^{j}$ are disjoint for $\lambda \neq \mu$, the images $q_{i}\left(\bar{P}_{\underline{\lambda}^{j}}^{j}\right)$ and $q_{i}\left(\bar{P}_{\mu}^{j}\right)$ intersect only along $E_{i}$. Imagine that we move $E_{i}$ inside $q_{i}\left(\bar{P}_{\mu}^{j}\right)$ a little bit and then intersect it with $q_{i}\left(\bar{P}_{\lambda}^{j}\right)$. All the intersections will be in $q_{i}\left(\bar{P}_{\lambda}^{j}\right) \cap q_{i}\left(\bar{P}_{\mu}^{j}\right)$, hence in $E_{i}$. Therefore $\left[E_{i}\right]\left[q_{i} \bar{P}_{\lambda}^{j}\right]$ is the same as the self-intersection of $E_{i}$ inside $q_{i} \bar{P}_{\mu}^{j}$. This latter is $B_{0} P_{\mu}^{j}$, so by 9.2 we get that $\left[E_{i}\right]\left[q_{i} \bar{P}_{\lambda}^{j}\right]=-1$.

(iii) It is quite easy to see that $H_{4}\left(C_{12}, \mathbf{R}\right)$ is generated by the classes $[E]$, $\left[\bar{P}_{\lambda}^{1}\right]$, and $\left[\bar{P}_{\lambda}^{2}\right]$. In $C_{12} \rightarrow C_{i}$ the class $[E]$ is mapped to zero. Thus $H_{4}\left(C_{i}, \mathbf{R}\right)$ is generated by $\left[P_{\lambda}^{i}\right]$ and $\left[q_{i} \bar{P}_{\lambda}^{j}\right] . p_{2}^{-1} p_{1}$ gives an isomorphism of $C_{1}-E_{1}$ and $C_{2}-E_{2}$, thus a natural identification of $H_{4}\left(C_{1}-E_{1}, \mathbf{R}\right)$ and $H_{4}\left(C_{2}-E_{2}, \mathbf{R}\right)$. Since real $\operatorname{dim} E_{i}=2$, we also have $H_{4}\left(C_{i}, \mathbf{R}\right) \cong H_{4}\left(C_{i}-E_{i}, \mathbf{R}\right)$, and so we get a natural isomorphism $t: H_{4}\left(C_{1}, \mathbf{R}\right) \rightarrow H_{4}\left(C_{2}, \mathbf{R}\right)$. This takes the class $\left[P_{\lambda}^{1}\right]$ to $\left[q_{2} \bar{P}_{\lambda}^{1}\right]$ and $\left[q_{1} \bar{P}_{\lambda}^{2}\right]$ to $\left[P_{\lambda}^{2}\right]$. Comparing this with our computations, we see that for any $a \in H_{4}\left(C_{1}, \mathbf{R}\right)$, we have $\left[E_{1}\right] a=-\left[E_{2}\right] t(a)$. 
This is indeed very interesting. If $F \subset X$ is a curve in a projective variety, then one cannot find an $F^{\prime}$ such that $[F] a=-\left[F^{\prime}\right] a$ for any $a \in H_{2 n-2}(X, \mathbf{R})$ since then $F \cup F^{\prime}$ would be homologous to zero.

In our example $E_{1}$ and $E_{2}$ are on different varieties, but these varieties are very close to each other. One can express this by saying that $C_{2}$ can be obtained from $C_{1}$ by a $\mathbf{C P} \mathbf{P}^{1}$ surgery: we remove $E_{1} \cong \mathbf{C P} \mathbf{P}^{1}$ from $C_{1}$ and replace it with $E_{2} \cong \mathbf{C P}^{1}$, the same curve but attached in a different way. This leads to the following:

12.2. GeNERAL SURGery PROBLEM. Let $X$ be a three-dimensional algebraic variety and $E \subset X$ be a curve. Can one find another algebraic variety $X^{\prime}$ and a curve $E^{\prime} \subset X^{\prime}$ such that $X-E$ and $X^{\prime}-E^{\prime}$ are isomorphic, but $X$ and $X^{\prime}$ are not? What freedom do we have in the choice of $X^{\prime}$ ? How can one compare $[E] \in H_{2}(X)$ and $\left[E^{\prime}\right] \in H_{2}\left(X^{\prime}\right)$.

We can start by considering the case when $X$ and $X^{\prime}$ are smooth, but if we want to use this for Mori's program, then we must allow at least terminal singularities.

It is not too hard to see that if both $X$ and $X^{\prime}$ have terminal (or even canonical) singularities, then there are maps $f: X \rightarrow Z$ and $f^{\prime}: X^{\prime} \rightarrow Z$ such that $f$ (resp. $f^{\prime}$ ) contracts $E$ (resp. $E^{\prime}$ ) to a point and is an isomorphism on $X-E$ (resp. $X^{\prime}-E^{\prime}$ ). Moreover, both $E$ and $E^{\prime}$ are isomorphic to $\mathbf{C P}^{1}$. Sometimes $Z$ will be a nonalgebraic variety (see 12.12 ) but this can be ignored for the moment. An argument similar to the one we made in 12.1 gives

$$
[E] c_{1}\left(K_{X}\right)=-\mu\left[E^{\prime}\right] c_{1}\left(K_{X^{\prime}}\right), \text { for some } \mu>0 \text {. }
$$

12.3. Definition. In the above situation it is worthwhile to distinguish three cases.

(i) $[E] c_{1}\left(K_{X}\right)=0$. Then $\left[E^{\prime}\right] c_{1}\left(K_{X}\right)=0$ again. The operation that produces $\left(X^{\prime}, E^{\prime}\right)$ from $(X, E)$ is called a flop of $E \subset X$. This is a symmetric operation.

(ii) $[E] c_{1}\left(K_{X}\right)<0$. Then $\left[E^{\prime}\right] c_{1}\left(K_{X^{\prime}}\right)>0$. The operation that produces $\left(X^{\prime}, E^{\prime}\right)$ from $(X, E)$ is called the flip of $(X, E)$. This is not a symmetric operation.

(iii) $[E] c_{1}\left(K_{X}\right)>0$. Then $\left[E^{\prime}\right] c_{1}\left(K_{X^{\prime}}\right)<0$. This is the inverse of the flip, so it might be called the inverse flip.

Given $(X, E)$ the flip or the inverse flip is unique. If $X$ is $\mathbf{Q}$-factorial, then the same holds for the flop.

The reason for this distinction is that these operations have different effects on the singularities. We will see that $X$ and its flop $X^{\prime}$ have essentially the same singularities along $E$ and $E^{\prime}$. If $X^{\prime}$ is the flip of $X$ then the singularities of $X^{\prime}$ along $E^{\prime}$ are simpler than the singularities of $X$ along $E$. Correspondingly the inverse flip creates more complicated singularities.

The basic existence result about flops is due to Reid:

12.4. THEOREM. Let $X$ be an algebraic threefold with terminal singularities. Let $\mathbf{C P}^{1} \cong E \subset X$ be a curve. Assume that $[E] c_{1}\left(K_{X}\right)=0$ and that $E$ can be contracted by a map $h: X \rightarrow Z$. Then a flop $\left(X^{\prime}, E^{\prime}\right)$ exists. 
Proof. The following proof is due to Mori. First we prove that $Z$ has terminal singularities. $K_{X}^{\otimes m}$ is trivial along $E$ so as in the proof of 11.13 we see that $K_{Z}^{\otimes m}$ exists. Again as in 11.13 we see that $Z$ has terminal singularities. Now we use the structure theorem 11.10. Assume for simplicity that $Z$ is of type (i) or (ii). Case (iii) requires more care because of the group action.

The exceptional set of $h: X-\operatorname{Sing} X \rightarrow Z$ is a curve, hence by $8.13, Z$ is not smooth. Thus $Z$ is defined by an equation $f(x, y, z)+\operatorname{tg}(x, y, z, t)$ and $f(x, y, z)$ contains the term $x^{2}$. Using the four-variable version of 2.12 and the quadratic formula, we can choose new coordinates $x^{\prime}, y^{\prime}, z^{\prime}, t^{\prime}$ such that $Z$ is given by an equation

$$
x^{\prime 2}+F\left(y^{\prime}, z^{\prime}, t^{\prime}\right)=0 .
$$

$T:\left(x^{\prime}, y^{\prime}, z^{\prime}, t^{\prime}\right) \rightarrow\left(-x^{\prime}, y^{\prime}, z^{\prime}, t^{\prime}\right)$ gives a map $T: Z \rightarrow Z$; let $U \subset Z$ be a $T$-invariant neighborhood of the origin. Since $f^{-1}(U)-E \cong U-0$, we have a map $T: f^{-1}(U)-E \rightarrow f^{-1}(U)-E$. One can see that this map will not extend to a regular map from $f^{-1}(U)$ to $f^{-1}(U)$.

$X$ is the union of $f^{-1}(U)$ and $X-E$ attached along $f^{-1}(U)-E$. Let $X^{\prime}$ be the union of $f^{-1}(U)$ and $X-E$ attached along $f^{-1}(U)-E$ in such a way that $u \in f^{-1}(U)$ is identified with $T(u) \in X-E$. The natural map $X \rightarrow X^{\prime}$ is not regular along $E$ since $T$ is not.

In 12.1 $C=Z$ and its equation $x y-u v=0$ has to be rewritten as $x^{\prime 2}-y^{\prime 2}$ $-u v=0$, where $x^{\prime}=(x+y) / 2, y^{\prime}=(x-y) / 2$.

12.5. EXAMPLE OF FLIP. Using 12.1 one can get the simplest example of flip as follows. On $C^{0}=(x y-u v=0)$ we define a $\mathbf{Z}_{2}$-action by $(x, y, u, v) \rightarrow$ $(x,-y, u,-v)$. One can easily see that this defines a $\mathbf{Z}_{2}$-action on $C_{12}, C_{1}$ and $C_{2}$.

The family of planes $P_{\lambda}^{2}$ has two members invariant under $\mathbf{Z}_{2}$. One is $P_{\infty}^{2}$, fixed pointwise; the other is $P_{0}^{2}$ where the action is -id. Using this, one can see that on $C_{1}$ the fixed points are exactly the points of $q_{1} \bar{P}_{\infty}^{2}$; on $C_{2}$ the fixed points are $E_{2} \cap P_{0}^{2}$ and the points of $P_{\infty}^{2}$.

If $M$ is a smooth threefold with a $\mathbf{Z}_{2}$-action, then the singularities of $M / \mathbf{Z}_{2}$ can come only from the fixed points. Locally at a fixed point, the action is like $\mathbf{C}^{3} /($ a linear action). There are three nontrivial cases:

(i) $(x, y, z) \rightarrow(x, y,-z)$. The fixed point set is two-dimensional and $\mathbf{C}^{3} / \mathbf{Z}_{2}$ $\cong \mathbf{C}^{3}$ via $(x, y, z) \rightarrow\left(x, y, z^{2}\right)$.

(ii) $(x, y, z) \rightarrow(x,-y,-z)$. The fixed point set is a line and $\mathbf{C}^{3} / \mathbf{Z}_{2}$ is isomorphic to $\left(u_{2} u_{3}-u_{4}^{2}=0\right) \subset \mathbf{C}^{4}$ via $(x, y, z) \rightarrow\left(x, y^{2}, z^{2}, y z\right)$.

(iii) $(x, y, z) \rightarrow(-x,-y,-z)$. The fixed point is isolated and $\mathbf{C}^{3} / \mathbf{Z}_{2}$ has an isolated singularity that we encountered in 10.16(iv)(e).

Applying this to $C_{i} / \mathbf{Z}_{2}$, we see that $C_{1} / \mathbf{Z}_{2}$ is smooth and one can compute that $E_{1} \subset C_{1} / \mathbf{Z}_{2}$ has +1 intersection with the canonical bundle. $C_{2} / \mathbf{Z}_{2}$ picks up an isolated singularity at the image of $E_{2} \cap P_{0}^{2}$. Here $K_{C_{2} / \mathbf{Z}_{2}}$ does not exist, and one can compute that $\left[E_{2} / \mathbf{Z}_{2}\right] c_{1}\left(K_{C_{2} / \mathbf{Z}_{2}}\right)=-1 / 2 . E_{2} / \mathbf{Z}_{2}$ generates a negative extremal ray in $C_{2} / \mathbf{Z}_{2}$ and the natural map $C_{2} / \mathbf{Z}_{2} \rightarrow C / \mathbf{Z}_{2}$ is a small contraction. $C_{1} / \mathbf{Z}_{2} \rightarrow C / \mathbf{Z}_{2}$ is the flip.

As we noted, $C_{2} / \mathbf{Z}_{2}$ is singular but $C_{1} / \mathbf{Z}_{2}$ is smooth. So the flip really improved the singularities. 
In order to use flips in the completion of Mori's program we need the following

12.6. Proposition. Let $X$ be a threefold with canonical singularities and $f$ : $X \rightarrow Y$ be a small contraction. Then there are curves $E_{i} \subset X$ such that $E_{i} \cong \mathbf{C P}^{1}$, $\left[E_{i}\right] c_{1}\left(K_{X}\right)<0, f\left(E_{i}\right)=$ point and $f$ is an isomorphism on $X-\cup E_{i}$.

Reid suggested flipping these curves $E_{i}$ and at each step introducing a curve $E_{i}^{\prime}$ with $\left[E_{i}^{\prime}\right] c_{1}\left(K_{X^{\prime}}\right)>0$. Unfortunately the flip of $E_{2}$ might affect $E_{1}^{\prime}$ and it is not clear how to flip all the $E_{i}$ 's at once. This vexing problem was overcome by Shokurov, who proved that any sequence of flips must terminate after finitely many steps.

12.7. Definition. The resulting $f^{\prime}: X^{\prime} \rightarrow Y$ is called the flip of the contraction map $f$.

The existence of the flips turns out to be a very difficult problem. Important special cases were settled by Tsunoda, Shokurov, Mori, and Kawamata; finally the general case was treated by Mori, who proved:

12.8. Third Fundamental Theorem. Let $X$ be a projective variety with terminal (or canonical) singularities and let $f: X \rightarrow Y$ be a small contraction. Then $f$ can be flipped.

Mori's method is very computational, but it gives a good geometric description of small contractions.

This completes Mori's program, at least in dimension three. Its two main consequences are the three-dimensional analogs of 10.11 and 10.14 . We postpone the former to the next chapter.

12.9. TheOREM. Let $g: X \rightarrow Z$ be a regular birational map between smooth projective threefolds. Then $g$ is the composite of divisorial contractions and flips.

This can be deduced from 13.1 the same way as 10.14 was deduced from 10.11 .

Note that although $g$ is regular, we might need some flips, which are nonregular. Examples show that in general $g$ cannot be written as a composite of divisorial contractions.

12.10. REMARK. It is possible that in the above situation $g$ can be written as the composite of divisorial contractions and flops. Since flops are much easier to understand than flips, this would be a stronger result.

Next we discuss an interesting problem which I have been deliberately avoiding so far. It is the unpleasant fact that flips frequently lead to nonalgebraic varieties. Again it is easier to give an example of flops.

12.11. Example. This will be based on the example given in 12.1, whose notation will be used.

Let $L=\mathcal{O}_{\mathbf{C P}^{4}}(1) \mid C$ be a line bundle. Let $s: C \rightarrow L^{\otimes 2}$ be a general section. We take a "square root" out of $s$ : this will be the set of points $V \subset L$ such that $v \otimes v \in s(C)$. Let $g: V \rightarrow C$ be the natural projection. If $x \in C$, and $s(x) \neq 0$, then $g^{-1}(x)$ consists of two points; if $s(x)=0$, then $g^{-1}(x)$ consists of one point. I may assume that the set of zeroes of $s$ in $C$ is a smooth subvariety which does not contain $0 \in C$. Then $V$ will be smooth outside 
$g^{-1}(0)$. I would like to find resolutions of $V$. Let $\left\{0_{1}, 0_{2}\right\}=g^{-1}(0)$. Near $0_{i} g$ is an analytic isomorphism. Thus at $0_{i}$ I can mimic either of the resolutions $C_{1}$ and $C_{2}$ of $C$. Let $p_{i j}: V_{i j} \rightarrow V$ be the resolution $I$ get by mimicking $C_{i}$ at $0_{1}$ and $C_{j}$ at $0_{2}$. Obviously there are natural maps $g_{i}: V_{i i} \rightarrow C_{i}$, but no such maps for $V_{i j}$, if $i \neq j$. Finally let $p_{i j}^{-1}\left(0_{k}\right)=E_{l}^{k}$, where $l=i$ if $k=1, l=j$ if $k=2$.

Let us study $V_{11}$ and the two exceptional curves $E_{1}^{1}$ and $E_{1}^{2}$. By a theorem of Lefschetz, $H_{4}\left(V_{11}, \mathbf{R}\right)$ is generated by $\left[p_{11}^{-1}\left(P_{\lambda}^{1}\right)\right]$ and $\left[p_{11}^{-1}\left(q_{1} \bar{P}_{\lambda}^{2}\right)\right]$. From our construction it is clear that $\left[E_{1}^{1}\right]$ and $\left[E_{1}^{2}\right]$ have the same intersection numbers with these classes in $H_{4}$, and therefore we conclude that $\left[E_{1}^{1}\right]=\left[E_{1}^{2}\right]$.

In $V_{12}$ the exceptional curves are instead $E_{1}^{1}$ and $E_{2}^{2}$; one can obtain the latter as a flop of $E_{1}^{2}$. Therefore $\left[E_{1}^{1}\right]=-\left[E_{2}^{2}\right] \in H_{2}\left(V_{12}, \mathbf{R}\right)$. Hence $\left[E_{1}^{1} \cup E_{2}^{2}\right]$ $=0 \in H_{2}\left(V_{12}, \mathbf{R}\right)$. By 6.6 this means that $V_{12}$ is a nonprojective complex manifold, and the same holds for $V_{21}$. On the other hand, both $V_{11}$ and $V_{22}$ are projective. Thus if one wants to flop $E_{1}^{2}$ and get a projective variety, then one has to flop $E_{1}^{1}$ simultaneously, and then we get $V_{22}$ which is projective.

12.12. EXAMPLE. The map $p_{11}: V_{11} \rightarrow V$ has another interesting property. This contracts the two disjoint curves $E_{1}^{i}$. At least analytically one can contract them one at a time, and obtain a factorization $t_{i}: V_{11} \rightarrow V_{i} \rightarrow V$, where $V_{11} \rightarrow V_{i}$ contracts the curve $E_{1}^{j}$ for $j \neq i$. Since

$$
\left[t_{1} E_{1}^{1}\right]=t_{1}\left[E_{1}^{1}\right]=t_{1}\left[E_{1}^{2}\right]=\left[t_{1} E_{1}^{2}\right]=[0]=0 \in H_{2}(V, \mathbf{R}),
$$

this $V_{1}$ is again not projective. However, it is glued together from two algebraic pieces $V_{1}-E_{1}^{1}$ and $V_{1}-0_{2}$; then it is a so-called abstract algebraic variety containing a curve homologous to zero. This cannot happen for smooth abstract algebraic varieties.

12.13. These examples show that unless we are willing to flop or flip several curves simultaneously we have to put up with nonprojective varieties. It is not completely clear to me which is better. This again points to the problem of finding an analog of Mori's program for nonprojective varieties. Not much is known about it.

13. Finer structure theory. Mori's program should be viewed as the beginning of a structure theory of algebraic varieties. In each birational equivalence class it finds some especially nice members. From this point of view the following is the main result of Mori.

13.1. THEOREM ABOUT CONVENIENT MODELS. Let $X^{\prime}$ be a projective variety of dimension at most three. Then $X^{\prime}$ is birationally equivalent to an $X$ which is Q-factorial, projective, has terminal singularities only, and is such that one of the following holds:

(i) $X$ admits a Fano contraction $f: X \rightarrow Y$, or

(ii) $K_{X}$ is nef.

This $X$ is not unique, but for a given $X^{\prime}$ only one of these possibilities can occur.

Proof. We construct a sequence of varieties $X_{1}, X_{2}, \ldots$ as follows. Let $X_{1}=X^{\prime}$. Assume that $X_{1}, \ldots, X_{i}$ are already constructed. If $K_{X_{t}}$ is nef, then we take $X=X_{i}$ and we are done. Otherwise, there is a contraction $g: X_{i} \rightarrow Z$. If this is a Fano contraction then we take $X=X_{i}, Y=Z$ and again we are 
done. If $g$ is a divisorial contraction, then we take $X_{i+1}=Z$. If $g$ is a small contraction, then we flip $g$ to get $g^{\prime}: X_{i}^{\prime} \rightarrow Z$ and we take $X_{i+1}=X_{i}^{\prime}$.

Each of the $X_{i}$ 's is $\mathbf{Q}$-factorial, projective, has terminal singularities and is birationally equivalent to $X$. If $X_{i} \rightarrow X_{i+1}$ is a divisorial contraction, then one can see that $\operatorname{dim} H_{4}\left(X_{i+1}, \mathbf{R}\right)=\operatorname{dim} H_{4}\left(X_{i}, \mathbf{R}\right)-1$. If $X_{i} \rightarrow X_{i+1}$ is a flip, then $\operatorname{dim} H_{4}\left(X_{i+1}, \mathbf{R}\right)=\operatorname{dim} H_{4}\left(X_{i}, \mathbf{R}\right)$. By the result of Shokurov (see 12.6), any sequence of flips is finite; thus our procedure most stop with some $X_{n}$. Thus either $X_{n}$ admits a Fano contraction or $K_{X_{n}}$ is nef.

If $X_{n}$ admits a Fano contraction, then $X_{n}$ is covered by rational curves (11.13(i)) and so is $X$. If $K_{X_{n}}$ is nef, then $X_{n}$ and $X$ cannot be covered by rational curves (8.25). Thus we can tell in advance whether we end up in the Fano contraction case or not.

Now the next task is to develop a structure theory for varieties admitting a Fano contraction and for varieties with $K_{X}$ nef. For surfaces a very satisfactory theory was developed by the old Italian school, and perfected subsequently by several geometers.

We already gave a complete description of the Fano contraction case in 10.10. Therefore we turn to the case $K_{X}$ nef. Note that $X$ is unique in its birational class by 9.11 . A short summary of the results is the following:

13.2. STRUCTURE OF SURFACES WITH $K_{X}$ NEF. Let $X$ be a smooth projective surface with $K_{X}$ nef. Then the stable canonical map is regular, and so the Iitaka variety is defined up to isomorphism. We have the following cases:

(i) $\kappa(X)=0, I(X)=$ point. There is a complete list of such varieties. They are $\mathbf{C}^{2} / L$, degree-four surfaces in $\mathbf{C P}^{3}$, and some closely related examples.

(ii) $\kappa(X)=1, \phi: X \rightarrow I(X)$ is a map onto a curve. All but finitely many of the fibers are smooth elliptic curves. The possibilities for other fibers are all known. $X$ can be completely described in terms of $I(X)$ and some additional structure on $I(X)$.

(iii) $\kappa(X)=2$. $\phi: X \rightarrow I(X)$ is birational; $I(X)$ has only canonical singularities. There is a plurigenus formula

$$
P_{m}(X)=\left(\frac{m(m-1)}{2}+\frac{1}{12}\right) c_{1}\left(K_{X}\right) \cdot c_{1}\left(K_{X}\right)+\frac{1}{12} e(X) \text { for } m \geqslant 2
$$

where $e(X)$ is the topological Euler characteristic. There are further results, the deepest one being $c_{1}\left(K_{X}\right) \cdot c_{1}\left(K_{X}\right) \leqslant 3 e(X)$.

13.3. Corollary. For a smooth projective surface the following are equivalent:

(i) $X$ is covered by rational curves;

(ii) $X$ is birational to $E \times \mathbf{C P}^{1}$ for some curve $E$;

(iii) $P_{m}(X)=0$ for every $m$;

(iv) $P_{12}(X)=0$.

Proof. We already noted in 8.25 that (i) $\Rightarrow$ (iii). If $P_{m}(X)=0$ for every $m$, then by $13.2 X$ cannot be birational to a surface with $K$ nef. Thus it is birational to a surface having a Fano contraction, hence (ii). (iv) needs a more detailed analysis of the cases in 13.2. 
For threefolds, of course, much less is known. There is no complete description of the Fano contractions although, as we already noted in 11.13(i), in this case $X$ is covered by rational curves (Miyaoka-Mori).

In the case when $K_{X}$ is nef, $X$ need not be unique. If $X$ contains a curve $E$ that can be flopped to get $X^{\prime \prime}$, then $K_{X^{\prime \prime}}$ is again nef. This situation was first studied by Kulikov in a special case and later in increasing generality by several other mathematicians (Friedman, Kawamata, Kollár, Mori, D. Morrison, Persson, Pinkham, Reid, Shepherd-Barron, Tsunoda). Now we have the following:

13.4. Theorem. Let $X$ and $X^{\prime}$ be Q-factorial projective threefolds with terminal singularities. Assume that $K_{X}$ and $K_{X^{\prime}}$ are nef. Let $f: X \rightarrow X^{\prime}$ be a birational map. Let $E \subset X$ be the set where $f$ is not defined. Then

(i) $E$ is a union of finitely many rational curves $E=\bigcup E_{i}$ and $\left[E_{i}\right] c_{1}\left(K_{X}\right)=0$ for every $i$.

(ii) At least one of the $E_{i}$ 's can be flopped.

(iii) $f$ can be written as the composite of finitely many flops.

For the case when $K_{X}$ is nef, the fundamental result is the following theorem of Miyaoka:

13.5. THEOREM. Let $X$ be a threefold with terminal (or canonical) singularities. Assume that $K_{X}$ is nef. Then $\kappa(X) \geqslant 0$; i.e., $P_{m}(X)>0$ for some $m>0$.

This yields the following analog of 13.3 .

13.6. Characterization of $\kappa=-\infty$. Let $X$ be a smooth projective threefold. The following are equivalent.

(i) $X$ is covered by rational curves;

(ii) $X$ is birational to a threefold admitting a Fano contraction;

(iii) $P_{m}(X)=0$ for every $m \geqslant 1$.

For the classes $\kappa \geqslant 0$ there is more information. Let $X$ be a projective threefold with terminal singularities and $K_{X}$ nef. We treat cases according to $\kappa(X)$.

13.7. $\kappa(X)=0$. There is a complete structure theory if $H_{1}(X, \mathbf{R}) \neq 0$. Essentially nothing is known if $H_{1}(X, \mathbf{R})=0$.

13.8. If $\kappa(X) \geqslant 1$, then the methods developed to prove 11.11 give that the stable canonical map is regular, and the litaka variety is defined up to isomorphism (Kawamata). Case by case information is as follows.

13.9. $\kappa(X)=1$. $\phi: X \rightarrow I(X), I(X)$ is a smooth curve. The smooth fibers of $\phi$ are surfaces from (i) of 13.2. Singular fibers are not fully understood.

13.10. $\kappa(X)=2$. $\phi: X \rightarrow I(X) . I(X)$ is a possibly singular surface; the possible singularities are more or less understood. Smooth fibers are elliptic curves; the singular ones are not fully understood.

13.11. $\kappa(X)=3$. $\phi: X \rightarrow I(X)$ is birational and $I(X)$ has canonical singularities. There is a plurigenus formula developed by Reid and his students. It is the sum of a cubic polynomial and of a periodic function, and the geometric meaning of the various terms is well understood. 
13.12. EPILOGUE. Before the appearance of the works of Mori, it was generally assumed that algebraic threefolds are in a state of considerable disorder and there is no hope of developing a theory analogous to that of algebraic surfaces. $M$. Noether said once that algebraic curves were created by God, algebraic surfaces by the Devil. This left little room for algebraic threefolds. I hope that I have succeeded in convincing the reader that there is a very deep and meaningful theory of algebraic threefolds which parallels the theory of algebraic surfaces. It is the hope of everyone working in this field that the results proved so far are only the beginning of a detailed structure theory.

\section{BIBLIOGRAPHY}

\section{INTRODUCTORY BOOKS}

C. H. Clemens, A scrapbook of complex curve theory, Plenum Press, 1980.

Elementary, unusual, and beautiful introduction to curves.

W. Fulton, Algebraic curves, W. A. Benjamin, 1969.

Leisurely algebraic introduction to curves.

D. Mumford, Algebraic geometry I: Complex projective varieties, Springer, 1976.

Using a little commutative algebra, this is the fastest way to interesting theorems. Part II does not exist.

M. Reid, Undergraduate algebraic geometry, London Math. Soc. Texts in Math. (to appear).

The most elementary introduction to the general theory.

I. R. Shafarevich, Basic algebraic geometry, Springer, 1977.

Parts I and II provide a peaceful algebraic introduction. Part III is an essentially independent glimpse at compact complex manifolds.

C. L. Siegel, Topics in complex function theory. I, II, III, Wiley-Interscience, 1969.

A beautiful introduction to the analytic theory of Riemann surfaces.

\section{MORE ADVANCED TEXTS}

W. Barth, C. Peters and A. Van de Ven, Compact complex surfaces, Springer, 1984. Comprehensive and elegant treatment of surfaces.

P. Griffiths and J. Harris, Principles of algebraic geometry, Wiley-Interscience, 1978.

A long and thorough introduction to the analytic theory of algebraic varieties.

R. Hartshorne, Algebraic geometry, Springer, 1977.

This is the standard algebraic introduction.

$\mathrm{K}$. Ueno, Classification theory of algebraic varieties and compact complex spaces, Lecture Notes in Math., vol. 439, Springer, 1975.

An overview prior to Mori's work.

\section{RECENT SURVEY ARTICLES}

C. H. Clemens, Curves on threefolds, the Abel-Jacobi mapping, Proc. Sympos. Pure Math. (Algebraic Geometry, Bowdoin College, 1985), Amer. Math. Soc., to appear.

Detailed study of the geometry of special threefolds. (The other point of view from Chapter 8.)

Y. Kawamata, K. Matsuda and K. Matsuki, Introduction to the minimal model problem, Adv. Studies in Pure Math., Sendai, to appear.

Comprehensive survey, aimed at experts.

S. Mori, Classification of higher-dimensional varieties, Proc. Sympos. Pure Math. (Algebraic Geometry, Bowdoin College, 1985), Amer. Math. Soc., to appear.

Mostly about results not encompassed by Mori's program. 
M. Reid, Tendencious survey of 3-folds, Proc. Sympos. Pure Math. (Algebraic Geometry, Bowdoin College, 1985), Amer. Math. Soc., to appear.

Mostly philosophy and jokes. Recommended for algebraic geometers.

M. Reid, Young person's guide to canonical singularities, Proc. Sympos. Pure Math. (Algebraic Geometry, Bowdoin College, 1985), Amer. Math. Soc., to appear.

Excellent introduction to three-dimensional terminal singularities.

P. M. H. Wilson, Towards birational classification of algebraic varieties, Bull. London Math. Soc. 19 (1987), 1-48.

Detailed survey aimed at the general algebraic geometer.

Department of Mathematics, Harvard University, Cambridge, Massachusetts 02138

Current address: Department of Mathematics, University of Utah, Salt Lake City, Utah 84112 
